\title{
Distal Anterior Cerebral Artery Aneurysms
}

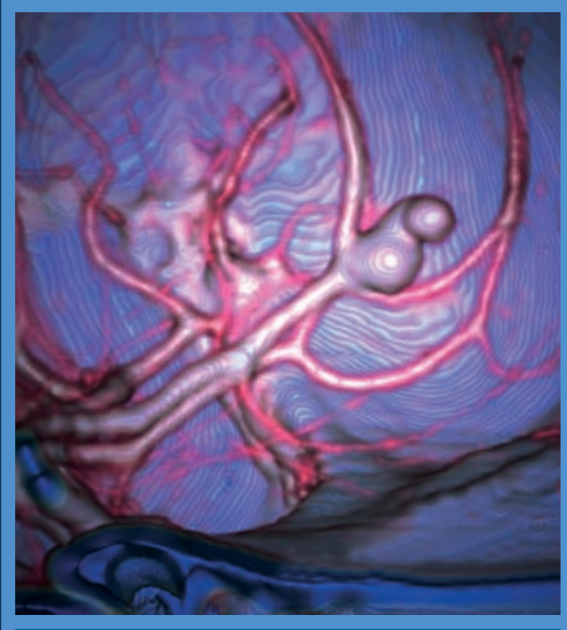

Martin Lehečka
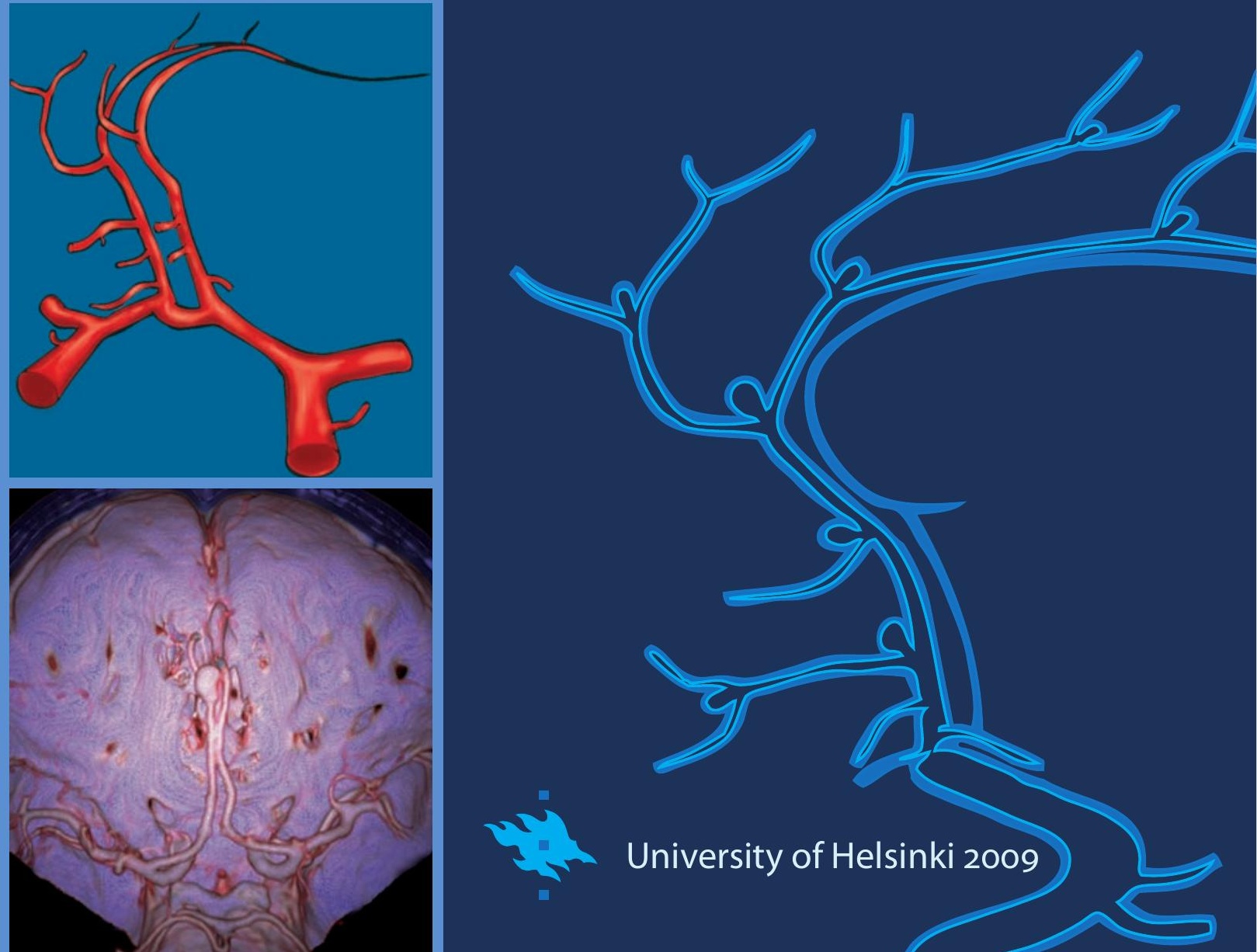
From the Department of Neurosurgery

Helsinki University Central Hospital

University of Helsinki

Helsinki, Finland

\section{Distal Anterior Cerebral Artery Aneurysms}

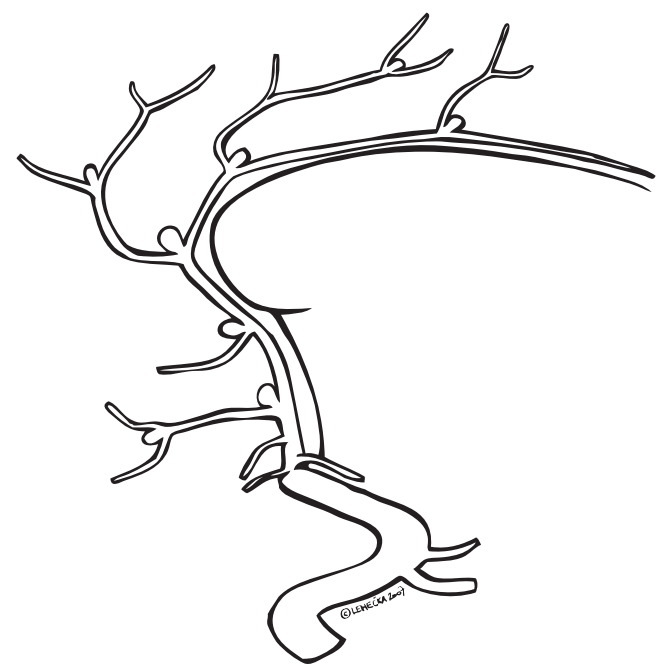

Martin Lehečka

Academic Dissertation

To be presented with the permission

of the Faculty of Medicine of the University of Helsinki for public discussion in the Lecture hall 1 of Töölö Hospital on February $6^{\text {th }}, 2009$ at 12 o'clock noon. 


\section{Supervised by:}

Professor Juha Hernesniemi, M.D., Ph.D.

Department of Neurosurgery

Helsinki University Central Hospital

Helsinki, Finland

Associate Professor Mika Niemelä, M.D., Ph.D.

Department of Neurosurgery

Helsinki University Central Hospital

Helsinki, Finland

\section{Reviewed by:}

Professor Juha Öhman, M.D., Ph.D.

Department on Neurosurgery

Tampere University Hospital

Tampere, Finland

Associate Professor Timo Kumpulainen, M.D., Ph.D.

Department of Neurosurgery

Oulu University Hospital

Oulu, Finland

\section{To be discussed with:}

Professor Robert F. Spetzler, M.D.

Director, Barrow Neurological Institute

J.N. Harber Chairman of Neurological Surgery

Professor, Section of Neurosurgery, University of Arizona

Phoenix, Arizona

$1^{\text {st }}$ edition 2009

(c) Martin Lehečka 2009

Cover and illustrations $\odot$ Martin Lehečka 2009

ISBN 978-952-92-4914-5 (paperback)

ISBN 978-952-10-5176-0 (PDF)

http://ethesis.helsinki.fi/

Helsinki University Press

Helsinki

2009 
To my grandfather 


\section{Author's contact information:}

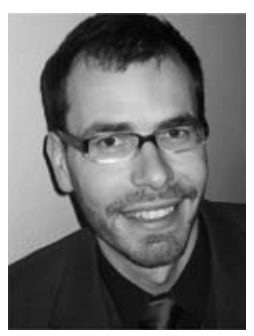

\section{Martin Lehečka}

Department of Neurosurgery

Helsinki University Central Hospital

Topeliuksenkatu 5

00260 Helsinki

Finland

mobile: +358504272500

fax: +358947187560

e-mail: martin.lehecka@hus.fi 


\section{Table of contents}

$\begin{array}{ll}\text { Abstract } & 11\end{array}$

$\begin{array}{ll}\text { Abbreviations } & 12\end{array}$

$\begin{array}{ll}\text { List of original publications } & 13\end{array}$

$\begin{array}{ll}\text { 1. Introduction } & 14\end{array}$

2. Review of literature $\quad 15$

2.1. INTRACRANIAL ANEURYSMS

2.1.1. Prevalence of intracranial aneurysms

2.1.2. Pathobiology of intracranial aneurysms $\quad 15$

2.1.2.1. Morphology and formation of intracranial aneurysms $\quad 15$

2.1.2.2. Genetics of intracranial aneurysms

2.1.2.3. Histology of intracranial aneurysms $\quad 15$

2.1.3. Subarachnoid hemorrhage 16

2.1.3.1. Incidence of SAH $\quad 16$

2.1.3.2. Natural history of ruptured intracranial aneurysms 16

2.1.4. Treatment of ruptured intracranial aneurysms 16

2.1.4.1. Rebleeding 16

2.1.4.2. Cerebral vasospasm 17

2.1.4.3. Other complications of SAH

2.1.5. Outcomes for ruptured IAs

$\begin{array}{ll}\text { 2.1.5.1. Outcome assessment } & 17\end{array}$

2.1.5.2. Treatment outcome 17

$\begin{array}{ll}\text { 2.1.5.3. Predictors for outcome after SAH } & 17\end{array}$

$\begin{array}{ll}\text { 2.1.6. Long-term follow-up after SAH } & 18\end{array}$

2.1.6.1. De novo aneurysms and rebleeding 18

2.1.6.2. Long-term mortality 18

2.1.7. Management of unruptured IAs $\quad 18$

2.2. HISTORY OF INTRACRANIAL ANEURYSM TREATMENT 19

2.2.1 Before microneurosurgery 19

2.2.1.1. Intracranial aneurysms in historical context 19

2.2.1.2. Hunterian ligation $\quad 19$

2.2.1.3. Carotid occlusion 20

2.2.1.4. Cerebral angiography 20

2.2.1.5. Wrapping and trapping $\quad 21$

2.2.1.6. Clipping $\quad 21$

2.2.1.7. Aneurysm clip development $\quad 21$

2.2.2. Microneurosurgery $\quad 22$

2.2.2.1. Operating microscope $\quad 22$

2.2.2.2. Development of microneurosurgery $\quad 22$

2.2.2.3. Other technical developments 
2.2.2.4. Microneurosurgery applied to intracranial aneurysms

2.2.3. Endovascular surgery

2.2.3.1. Balloon occlusion

2.2.3.2. Coiling

2.2.4. Aneurysm surgery in Finland

2.2.4.1. Aarno Snellman, founder of Finnish neurosurgery

2.2.4.2. Angiography in Finland

2.2.4.3. World War II and the late $1940 \mathrm{~S}$

2.2.4.4. Initial steps in aneurysm surgery

2.2.4.5. Microneurosurgery and endovascular surgery

2.3. Microsurgical ANATOMY FOR DACA ANEURYSMS

2.3.1. Anterior cerebral artery

2.3.1.1. A1 segment of ACA 28

2.3.1.2. Anterior communicating artery $(A C O A)$ complex

2.3.1.3. Perforating branches of $A C O A$

2.3.1.4. A2 segment

2.3.1.5. Arterial branches of A2 segment

2.3.1.6. Recurrent artery of Heubner (RAH)

2.3.1.7. Orbitofrontal artery (OFA)

2.3.1.8. Frontopolar artery (FPA)

2.3.1.9. Basal perforating branches

2.3.1.10. A3 segment and its branches

2.3.1.11. Callosomarginal artery (CMA)

2.3.1.12. A4 and A5 segments and their cortical branches 32

2.3.1.13. Anatomic anomalies of ACA 32

2.3.2. Interhemispheric fissure 33

2.3.3. Venous structures $\quad 33$

2.3.4. Corpus callosum $\quad 34$

2.4. DISTAL ANTERIOR CEREBRAL ARTERY ANEURYSMS

2.4.1. Incidence and location of DACA aneurysms 35

2.4.1.1. Aneurysms of the ACA $\quad 35$

2.4.1.2. A2As (Proximal pericallosal aneurysms) $\quad 35$

2.4.1.3. A3As (Classical pericallosal aneurysms) 36

2.4.7.4. AdistAs (Distal pericallosal aneurysms) 36

2.4.2. Clinical symptoms 36

2.4.3. Anatomic features 36

2.4.3.1. Size 36

2.4.3.2. Multiple aneurysms

2.4.3.3. ICH and IVH

2.4.3.4. Association with ACA anomalies $\quad 37$

2.4.3.5. Association with AVMs 38

2.4.4. Special subgroups of DACA aneurysms 38

2.4.4.1. Giant DACA aneurysms 38

2.4.4.2. Aneurysms associated with azygos ACA 38

2.4.4.3. Traumatic DACA aneurysms 38 
2.4.5. Imaging of DACA aneurysms

2.4.6. Treatment of DACA aneurysms

2.4.6.1. History

40

2.4.6.2. Conservative treatment

2.4.6.3. Direct surgery before microneurosurgery

2.4.6.4. Microneurosurgery for DACA aneurysms

2.4.6.5. Technical difficulties in DACA aneurysm surgery

2.4.6.6. Endovascular treatment

2.4.6.7. Technical difficulties in coiling of DACA aneurysms

2.4.7. Outcome

2.4.7.1. Short-term outcome

2.4.7.2. Predictors of short-term outcome

46

2.4.7.3. Long-term outcome

\section{Aims of the study}

\section{Patients, materials and methods}

4.1. Publication I: Anatomic FEATURes of DACA ANeurysms 48

$\begin{array}{ll}\text { 4.1.1. Patients and images } & 48\end{array}$

4.1.2. Image analysis $\quad 49$

$\begin{array}{lr}\text { 4.1.3. Statistical analysis } & 49\end{array}$

4.2. Publication II: Treatment AND OUtCome of DACA Aneurysms 49

4.2.1. Patients $\quad 49$

4.2.2. Data collection $\quad 50$

4.2.3. Statistical analysis $\quad 50$

4.3. Publication III: LONG-TERM OUTCOME OF RUPTURED DACA ANEURYSMS 51

4.3.1. Patients

4.3.2. Follow-up $\quad 51$

4.3.3. Statistical analysis $\quad 51$

4.4. Publications IV-VI: Microneurosurgical management of DACA ANeURYsms 51

4.4.1. Patients (Kuopio Cerebral Aneurysm Database) 51

4.4.2. Analysis of microneurosurgical technique $\quad 51$

4.4.3. Microneurosurgical videos $\quad 52$

5. Results

5.1. INCIDENCE OF DACA ANEURYSMS (PUBLICATIONS IV-VI) 53

5.2. ANATOMY OF DACA ANEURYSMS (PUBLICATION I)

5.2.1. Microneurosurgical classification $\quad 53$

$\begin{array}{ll}\text { 5.2.2. Aneurysm size } & 54\end{array}$

5.2.3. Dome and base $\quad 54$

5.2.4. Multiple aneurysms $\quad 55$

5.2.5. Anomalies of ACA $\quad 56$

5.2.5.1. Azygos ACA $\quad 56$

5.2.5.2. Bihemispheric ACA 56

5.2.5.3. Triplication of $A C A \quad 56$ 
5.3. SAH FROM RUPTURED DACA ANEURYSM (PUBLICATION II, PART B)

5.3.1. Age, presentation, and clinical condition 57

5.3.2. Radiological features $\quad 57$

5.3.2.1. CT findings $\quad 57$

5.3.2.2. Angiographic findings (DSA, CTA, MRA) 58

5.4. TREATMENT AND OUTCOME OF DACA ANEURYSMS (PUBLICATION II) 58

5.4.1. Ruptured DACA aneurysms 1936-1979 (publication II, part a) 58

5.4.2. Ruptured DACA aneurysms 1980-2005 (publication II, part b) 58

5.4.2.1. Microneurosurgical clipping $\quad 58$

$\begin{array}{ll}\text { 5.4.2.2. Coiling } & 59\end{array}$

5.4.2.3. Outcome at one year 60

5.4.2.4. Factors predicting outcome at one year 60

5.4.3. Unruptured DACA aneurysms 1980-2005 (publication II, part b) 61

5.4.3.1. Unruptured DACA aneurysms without acute SAH $\quad 61$

$\begin{array}{ll}\text { 5.4.3.2. Unruptured DACA aneurysms with acute } S A H & 61\end{array}$

5.5. LONG-TERM FOLLOW-UP OF RUPTURED DACA ANEURYSMS (PUBLICATION III) 61

$\begin{array}{ll}\text { 5.5.1. Rebleeding } & 61\end{array}$

$\begin{array}{ll}\text { 5.5.2. Mortality } & 61\end{array}$

$\begin{array}{ll}\text { 5.5.2.1. Early mortality } & 61\end{array}$

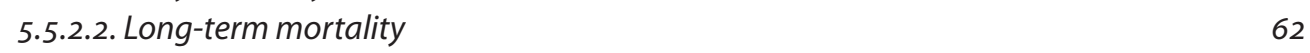

$\begin{array}{ll}\text { 5.5.3. Excess mortality } & 62\end{array}$

5.6. MICRONEUROSURGICAL TECHNIQUE FOR CLIPPING DACA ANEURYSMS (PUBLICATIONS IV-VI) 63

$\begin{array}{ll}\text { 5.6.1. Approach } & 63\end{array}$

5.6.2. Positioning, craniotomy and dural opening 64

$\begin{array}{ll}\text { 5.6.3. CSF drainage } & 64\end{array}$

5.6.4. Removal of ICH $\quad 65$

5.6.5. Dissection towards the aneurysm $\quad 65$

$\begin{array}{ll}\text { 5.6.6. Dissection of the aneurysm } & 68\end{array}$

$\begin{array}{ll}\text { 5.6.7. Temporary clipping } & 68\end{array}$

$\begin{array}{ll}\text { 5.6.8. Clipping of the aneurysm } & 68\end{array}$

$\begin{array}{ll}\text { 5.6.9. Intraoperative aneurysm rupture } & 69\end{array}$

5.6.10. Verification of clipping $\quad 69$

$\begin{array}{ll}\text { 5.6.11. Associated aneurysms } & 69\end{array}$

5.6.12. Special considerations for each DACA aneurysm location 69

5.6.12.1. Frontobasal A2As (publication IV) $\quad 69$

5.6.12.2. A2 trunk A2As (publication IV) 70

$\begin{array}{ll}\text { 5.6.12.3. Inferior } A 3 A s \text { (publication } V \text { ) } & 71\end{array}$

5.6.12.4. Anterior A3As (publication V) $\quad 72$

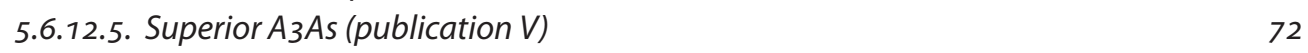

5.6.12.6. A4 and A5 aneurysms (publication VI) $\quad 73$

5.6.12.7. Distal branch AdistAs (publication VI) 74

6. Discussion $\quad 75$

$\begin{array}{ll}\text { 6.1. ANATOMIC FEATURES } & 75\end{array}$ 
6.1.1. DACA aneurysm locations

6.1.2. Aneurysm size and dome orientation $\quad 75$

$\begin{array}{ll}\text { 6.1.3. Aneurysm base and associated branches } & 75\end{array}$

6.1.4. ACA anomalies $\quad 76$

6.1.5. Associated aneurysms and AVMs $\quad 76$

6.1.6. ICHs from ruptured DACA aneurysms $\quad 77$

6.1.7. Imaging of DACA aneurysms and CTA $\quad 77$

6.2. TREATMENT OF DACA ANEURYSMS

$\begin{array}{ll}\text { 6.2.1. Clipping of DACA aneurysms } & 77\end{array}$

$\begin{array}{ll}\text { 6.2.2. Coiling of DACA aneurysms } & 78\end{array}$

$\begin{array}{ll}\text { 6.3. OUTCOME } & 78\end{array}$

$\begin{array}{ll}\text { 6.3.1. Ruptured DACA aneurysms } & 78\end{array}$

6.3.1.1. One year outcome $\quad 78$

6.3.1.2. Predictors of outcome at one year $\quad 79$

6.3.1.3. Neurological deficits $\quad 79$

6.3.2. Unruptured DACA aneurysms $\quad 79$

6.4. LONG-TERM FOLLOW-UP $\quad 80$

6.4.1. Rebleeding from clipped DACA aneurysms 80

6.4.2. Recurrent SAH and de novo aneurysms 80

$\begin{array}{lr}\text { 6.4.3. Mortality } & 80\end{array}$

6.4.4. Excess mortality $\quad 80$

6.4.5. Long-term angiographic screening $\quad 81$

6.5. FUTURE TRENDS

7. Conclusions $\quad \mathbf{8 4}$

List of 12 supplementary videos on microneurosurgery of DACA aneurysms $\quad 85$

Acknowledgments $\quad 86$

$\begin{array}{ll}\text { References } & 88\end{array}$ 


\section{Abstract}

Objective: Distal anterior cerebral artery (DACA) aneurysms represent about $6 \%$ of all intracranial aneurysms. So far, only small series on treatment of these aneurysms have been published. Our aim is to evaluate the anatomic features, treatment results, and long-term outcome of DACA aneurysms. In addition, we address the current techniques for microneurosurgical treatment of these lesions.

Patients and methods: We analyzed the clinical and radiological data on 517 consecutive patients diagnosed with DACA aneurysm at two neurosurgical centers serving solely the Southern (Helsinki) and Eastern (Kuopio) Finland in 1936-2007. We used a defined subgroup of the whole study population in each part of the study. Detailed anatomic analysis was performed in 101 consecutive patients from 1998 to 2007. Treatment results were analyzed in 427 patients treated between 1980 to 2005 , the era of CT imaging and microneurosurgery. With a median follow-up of 10 years we evaluated the long-term outcome of treatment in 280 patients with ruptured DACA aneurysm(s); no patients were lost to followup.

Results: DACA aneurysms, found most often $(83 \%)$ at the $A_{3}$ segment of the anterior cerebral artery (ACA), were smaller (median $6 \mathrm{~mm}$ vs. $8 \mathrm{~mm}$ ), more frequently associated with multiple aneurysms ( $35 \%$ vs. $18 \%$ ), and presented more often with intracerebral hematomas (ICHs) (53\% vs. $26 \%$ ) than ruptured aneurysms in general. They were associated with anomalies of the ACA in $23 \%$ of the patients. Microsurgical treatment showed similar complication rates (treatment morbidity 15\%, treatment mortality $0.4 \%$ ) as for other ruptured aneurysms. At oneyear after subarachnoid hemorrhage (SAH), DACA aneurysms had equally favorable outcome $(G O S \geq 4)$ as other ruptured aneurysms (74\% vs. $69 \%$ ) but their mortality was lower ( $13 \%$ vs. $24 \%$ ). Factors predicting unfavorable outcome for ruptured DACA aneurysms were advanced age, Hunt\&Hess grade $\geq 3$, rebleeding before treatment, $\mathrm{ICH}$, intraventricular hemorrhage, and severe preoperative hydrocephalus. The cumulative relative survival ratio showed $16 \%$ excess mortality in patients with ruptured DACA aneurysm during the first three years after SAH compared to the matched general population. From the fourth year onwards, there was no excess mortality during the followup. There were four episodes of recurrent $\mathrm{SAH}$, only one due to treated DACA aneurysm, with a 10-year cumulative risk of $1.4 \%$.

Conclusions: The special neurovascular features and frequent association with anterior cerebral artery anomalies must be taken into account when planning occlusive treatment of DACA aneurysms. With microneurosurgery, ruptured DACA aneurysms have equally favorable outcome but lower mortality at one year as ruptured aneurysms in general. Clipping of DACA aneurysms provides a long-lasting result, with very small rates of rebleeding. After surviving three years from rupture of DACA aneurysm, the long-term survival of these patients becomes similar to that of the matched general population. 


\section{Abbreviations}
A1 $=$ Proximal segment of anterior cerebral artery
$\mathrm{A}_{1} \mathrm{~A}=$ Aneurysm of the $\mathrm{A}_{1}$ segment of anterior cerebral artery
A2 = A2 segment of anterior cerebral artery
$\mathrm{A}_{2} \mathrm{~A}=$ Aneurysm of the $\mathrm{A} 2$ segment or frontobasal branch of anterior cerebral artery

A3 $=A_{3}$ segment of anterior cerebral artery

$\mathrm{A}_{3} \mathrm{~A}=$ Aneurysm of the $\mathrm{A}_{3}$ segment of anterior cerebral artery

A4 = A4 segment of anterior cerebral artery

A5 $=$ A5 segment of anterior cerebral artery

$\mathrm{ACA}=$ Anterior cerebral artery

$\mathrm{ACh} A=$ Anterior choroidal artery

$\mathrm{ACo} A=$ Anterior communicating artery

$\mathrm{ACoAA}=$ Anterior communicating artery aneurysm

Adist $\mathrm{A}=$ Aneurysm distal to $\mathrm{A} 3$ segment of anterior cerebral artery

AIFA = Anterior internal frontal artery

AVM = Arteriovenous malformation

$\mathrm{Cl}=$ Confidence interval

CMA = Callosomarginal artery

CRSR = Cumulative relative survival ratio

CSF $=$ Cerebrospinal fluid

$\mathrm{CT}=$ Computed tomography

CTA = Computed tomographic angiography

DACA = Distal anterior cerebral artery

DSA = Digital subtraction angiography

ENT = Ear Nose \& Throat

FPA = Frontopolar artery

GCC = Genu of corpus callosum

GCS = Glasgow coma scale

GDC = Guglielmi detachable coil

GOS = Glasgow outcome score

$\mathrm{H} \& \mathrm{H}=$ Hunt \& Hess grade

IA = Intracranial aneurysm

ICA = Internal carotid artery

ICG = Indocyanine green

$\mathrm{ICH}=$ Intracerebral hematoma
ISAT = International Subarachnoid Aneurysm Trial

ISUIA = International Study of Unruptured Intracranial Aneurysms

IVH = Intraventricular hemorrhage

LSO = Lateral supraorbital approach

MCA = Middle cerebral artery

MIFA = Middle internal frontal artery

MLA = Medial lenticulostriate arteries

MRA = Magnetic resonance angiography

MRI = Magnetic resonance imaging

OFA = Orbitofrontal artery

OR = Odds ratio

PerA = Pericallosal artery

PCA = Posterior cerebral artery

$\mathrm{PCOA}=$ Posterior communicating artery

PICA = Posterior inferior cerebellar artery

PIFA = Posterior internal frontal artery

$\mathrm{RAH}=$ Recurrent artery of Heubner

RSR = Relative survival ratio

$\mathrm{SAH}=$ Subarachnoid hemorrhage

$\mathrm{SD}=$ Standard deviation

SMA = Supplementary motor area

SMR = Standardized mortality ratio

STA = Superficial temporal artery 


\section{List of original publications}

This thesis is based on the following publications, referred to in the text by their Roman numerals:

I. Lehecka M, Porras M, Dashti R, Niemelä M, Hernesniemi J. Anatomic features of distal anterior cerebral artery aneurysms: a detailed angiographic analysis of 101 patients. Neurosurgery 2008, 63(2): 219-229.

II. Lehecka M, Lehto $H$, Niemelä $M$, Juvela S, Dashti R, Koivisto T, Ronkainen A, Rinne J, Jääskeläinen JE, Hernesniemi J. Distal anterior cerebral artery aneurysms: treatment and outcome analysis of 501 patients. Neurosurgery 2008, 62(3): 590-601 .

III. Lehecka M, Niemelä M, Seppänen J, Lehto H, Koivisto T, Ronkainen A, Rinne J, Sankila $\mathrm{R}$, Jääskeläinen JE, Hernesniemi J. No long-term excess mortality in 280 patients with ruptured distal anterior cerebral artery aneurysms. Neurosurgery 2007; 60(2): 235-241.

IV. Lehecka M, Dashti R, Hernesniemi J, Niemelä M, Koivisto T, Ronkainen A, Rinne J, Jääskeläinen JE. Microneurosurgical management of aneurysms at A2 segment of anterior cerebral artery (proximal pericallosal artery) and its frontobasal branches. Surg Neurol 2008, 70(3): 232-246.

V. Lehecka M, Dashti R, Hernesniemi J, Niemelä M, Koivisto T, Ronkainen A, Rinne J, Jääskeläinen JE. Microneurosurgical management of aneurysms at $A 3$ segment of anterior cerebral artery. Surg Neurol 2008, 70(2): 135-152.

VI. Lehecka M, Dashti R, Hernesniemi J, Niemelä M, Koivisto T, Ronkainen A, Rinne J, Jääskeläinen JE. Microneurosurgical management of aneurysms at $A_{4}$ and $A_{5}$ segments and distal cortical branches of anterior cerebral artery. Surg Neurol 2008, 70(4): 352-367.

The original publications are reproduced with permission of the copyright holders. 


\section{Introduction}

Intracranial aneurysms (IAs) are acquired dilatations of intracranial arteries. They are typically located at the arterial branching points near the skull base. When an IA ruptures, it causes subarachnoid hemorrhage (SAH). Typical symptoms include sudden onset of severe headache, nausea, vomiting and often loss of consciousness. Risk factors for SAH include smoking, excessive alcohol consumption, hypertension and familial history $[76,155,156]$. SAH is a devastating event associated with cumulative mortality up to $50 \%$ at six months $[84,131,381]$. $\mathrm{SAH}$ represents only about $5-10 \%$ of strokes, but because the disease strikes at a fairly young age $(\approx 50 \mathrm{yrs})$ and is often fatal, the loss of productive life years is similar to that for cerebral infarction and intracerebral hemorrhage [153]. The most important goal in the treatment of $\mathrm{SAH}$ patients is to prevent rebleeding from the ruptured aneurysm. At present, this is achieved by occluding the aneurysm either with microneurosurgical or endovascular methods.

Distribution of IAs along the different intracranial arteries is unequal so that certain arteries and their segments present more often with aneurysms than others, possibly due to flow related reasons. One of the infrequent aneurysm locations is the distal portion of the anterior cerebral artery (ACA), also called the pericallosal artery (PerA). Only about 6\% of all IAs are found on this artery or on one of its cortical branches $[52,122,142,205,248,279,312,368,383,438,445$, 449]. These aneurysms, located distally to the anterior communicating artery $(\mathrm{ACO} A)$ on the A2-A5 segments of the ACA and embedded between the cerebral hemispheres, are called distal anterior cerebral artery (DACA) aneurysms $[122,198]$. They have special features such as small size in concordance with the relatively small caliber of the DACA itself, and a broad base with originating branches, which have to be taken into account in their treatment, making especially endovascular therapy relatively difficult $[52,122,205,248,279,312,368,383,438$, $443,449]$. In addition, they are associated with vascular anomalies of the ACA, such as azygos, bihemispheric and triplicated pericallosal arteries, arteriovenous malformations (AVMs), and multiple aneurysms $[122,133,383,438]$.

Microneurosurgery has been the treatment of choice for DACA aneurysms for several decades, whereas endovascular treatment has been used less frequently for the reasons mentioned above and only over the past fifteen years [184,265,300]. Although clipping has been the gold standard, the microsurgical series on DACA aneurysms published so far are relatively small $[32,52,122,142,231,248,264,279$, $312,368,383,438,445]$. The rarity of DACA aneurysms combined with the fact that they require a different microsurgical approach than other anterior circulation aneurysms [122,142,443] reduce opportunities to gain experience in the management of these aneurysms.

This study presents the combined experience of two Finnish neurosurgical centers (Helsinki and Kuopio University Hospitals) with population responsibility (of close to 3 million people), in a consecutive, retrospective series of 517 patients with DACA aneurysms treated between 1936 and 2007. This series of DACA aneurysms is by far the largest published to date. The data are based on an ethnically homogenous population with good medical records and complete follow-up of all patients. The aim is to provide new information on the anatomic features, treatment, microneurosurgical techniques, outcome, and long-term follow-up of DACA aneurysms, also in comparison to the matched general population. 


\section{Review of literature}

\subsection{Intracranial aneurysms}

\subsubsection{Prevalence of intracranial aneurysms}

Intracranial aneurysms (IAs) are found in about $2 \%$ of the general adult population, and are considered to be acquired lesions [328]. In Finland (population 5.3 million) the standardized prevalence is estimated to be $2.2-3 \%$, implying that 100 o0o Finns would carry unruptured IAs [336]. Most of the aneurysms are saccular (97\%) and arise at sites of arterial branching [49]. Women are more likely to harbor an IA than men (relative risk = 1.3), and this proportion increases with age [328]. Also, in families with two or more members affected with IAs, the risk of having an IA is $2-4$ times higher than what is expected in a general western population [335]. Multiple aneurysms are usually found in $28-35 \%$ of patients with IAs $[49,50,70,140,331]$.

\subsubsection{Pathobiology of intracranial aneurysms}

\subsubsection{Morphology and formation of intracranial aneurysms}

IAs are usually divided into two groups based on their morphological features: (a) saccular aneurysms, which are pouch-like protrusions of the vessel wall usually found at the bifurcations of the intracranial arteries and comprise $97 \%$ of all IAs; and (b) fusiform aneurysms, which are dilatations of the whole arterial segment with neither a distinguishable base nor a separate pouch, comprising about $3 \%$ of all IAs $[47,48]$. A small number of fusiform aneurysms are dissecting in origin. Although the exact pathobiological mechanism of IA formation is still unknown, IAs are believed to be acquired lesions. Some are caused by direct vascular trauma (traumatic aneurysms) [19,261], or bacterial infection (mycotic aneurysms) [35,86,201], but in the vast majority of cases, a combination of multiple factors are probably involved, including hemodynamic stress acting on the vessel wall $[85,129]$, inflammation processes $[89,411]$, arterial wall remodeling and degeneration [88,203], and a multitude of extrinsic risk factors such as smoking, hypertension, alcohol consumption and genetic predisposition $[76,161,421]$, which cause the IA formation and growth.

\subsubsection{Genetics of intracranial aneurysms}

The concept of genetic factors being involved in the development of aneurysms has lead to many studies on the genetic determinants for IA. So far, different genome-wide linkage studies have identified several loci [344], but only four (1p34.3-p36.13, 7q11, 19q13.3 and Xp22) have been replicated in different populations [75,246,259,283,284,345,420,439]. Knowledge on the genetic determinants may provide insight into the development of aneurysms, and thereby give clues on how to stop aneurysm formation. It may also provide diagnostic tools for identifying individuals at increased risk for aneurysm formation who can be screened by imaging studies. In the near future, whole-genome mutation analysis will probably give more data.

\subsubsection{Histology of intracranial aneurysms}

Normal intracranial arteries are composed of three histological layers: loose connective tissue layer (adventitia); muscular layer of smooth muscle cells (media); and inner layer of endothelial cells and smooth muscle cells (intima) [341]. There is also a very thin layer of elastic fibers, the internal elastic lamina, at the border between the media and intima. IAs usually have a disorganized wall structure and lack elastic laminae $[175,196]$. Four dominant histo- 
logical wall types of IAs have been recognized: (A) endothelialized wall with linearly organized smooth muscle cells; (B) thickened wall with disorganized smooth muscle cells; (C) hypocellular wall with fresh or organizing thrombosis; and (D) extremely thin thrombosis-lined hypocellular wall [89]. Many aneurysm walls are heterogeneous, constituted by a combination of the different wall types, but the $C$ and $D$ types are predominant in ruptured aneurysms [89]. The histological differences between ruptured and unruptured IAs suggest that the aneurysm wall is a dynamic structure undergoing constant remodeling [88]. Complement activation seems to associate with degeneration and rupture of IAs [411]. Also protein kinases (c-Jun $\mathrm{N}$-terminal kinase and p38 kinase) are involved in the growth and rupture of IAs [203]. A better understanding of the molecular mechanisms behind IA formation and rupture may provide possibilities of targeted pharmacological therapy for IAs in the future.

\subsubsection{Subarachnoid hemorrhage}

Subarachnoid hemorrhage (SAH) due to rupture of an IA is a devastating event associated with high rates of morbidity and 50\% mortality [131]. SAH accounts for $5-10 \%$ of all strokes, but as the disease strikes at a fairly young age (mean age 50 years) the loss of productive life years can be significant $[153,190]$. Besides IAs (80-85\%), SAH can also be caused by AVMs (5\%), or an unknown etiology (15\%) $[172,334,353]$. In the latter case, the prognosis is very good.

\subsubsection{Incidence of SAH}

The incidence of SAH varies in different populations. In most populations the incidence is 6-10 cases per 100 ooo person-years $[7,218]$. For unknown reasons, probably genetic, in Finland, Japan and Northern Sweden the incidence is much higher with 16-20 cases per 100 ooo person-years [84,138,277,369,381].
This means that in Finland about 1000 patients suffer from SAH every year. With almost $50 \%$ mortality, more people in Finland die annually because of SAH than due to traffic accidents. In 2006, there were 365 deaths due to $\mathrm{SAH}$ compared to 307 deaths from traffic accidents [379].

\subsubsection{Natural history of ruptured intracranial aneurysms}

About $15 \%$ of the SAH patients die before reaching medical attention [132,290,301]. In the historical, unselected series by Pakarinen the cumulative mortality was $32 \%$ during the first day, 46\% during the first week, 56\% during the first month and $60 \%$ during 6 months [290]. The initial hemorrhage causes the greatest mortality [84], being also the reason why even with the advent of new treatments the case-fatality rate of SAH has been declining very slowly $[131,369,381]$. If the aneurysm is left untreated, about one third of the patients who recover from the initial hemorrhage will die of rebleeding during the first 6 months [124,290,298]. Delayed cerebral vasospasm is the second major cause of death in patients surviving the initial ictus $[106,170]$. Even with modern treatment, case-fatality rates are still close to $50 \%$ at one month after SAH [131,301,369,381]

\subsubsection{Treatment of ruptured intracranial aneurysms}

Treatment of ruptured IAs focuses on three major issues: (a) to prevent rebleeding; (b) to prevent delayed vasospasm; and (c) to take care of all the additional problems caused by the initial impact of SAH.

\subsubsection{Rebleeding}

The peak incidence of rebleeding occurs during the first 24 hours when the risk is $4-7 \%$ $[141,158,171,290]$. After this the risk remains at $1-2 \%$ per day for the following two weeks, 
and for the first month the cumulative risk is $30-35 \%$. The problem with rebleeding is that about $60 \%$ of the patients who have a rebleeding die $[141,158,171,290]$. The best method to prevent rebleeding is intervention with either microneurosurgical or endovascular approach $[237,250]$. Early surgery combined with nimodipine (calcium antagonist) treatment has been shown to reduce both the rebleeding rate and the risk for vasospasm [278]. By preventing early rebleeds, early surgery both decreases the mortality rate and improves the quality of life of the survivors [84].

\subsubsection{Cerebral vasospasm}

Cerebral vasospasm is defined as delayed narrowing of intracranial arteries often associated with diminished perfusion in the territory of the affected artery ultimately leading to hypoxia [237]. Angiographic vasospasm is detected in $50-75 \%$ of the patients with a typical onset of three to five days after SAH $[139,170]$. Without treatment about half of these patients develop clinical symptoms of ischemic neurological deficits and some even die [125]. Combined mortality and morbidity associated with cerebral vasospasm is about $15 \%$ of all $\mathrm{SAH}$ patients $[106,222]$. So far, no single treatment to prevent vasospasm really effectively has been identified.

\subsubsection{Other complications of SAH}

Additional complications related to acute $\mathrm{SAH}$ include hydrocephalus, expansive intracerebral hematomas (ICHs), hyponatremia, seizures, and less frequently also cardiac arrhythmia, cardiac dysfunction, myocardial injury, pulmonary edema, acute lung injury, renal dysfunction, and hepatic dysfunction $[48,49,111$, $112,123,329,377,388,393,413]$. This shows how $\mathrm{SAH}$ not only affects the brain but also has an impact on almost the whole body.

\subsubsection{Outcomes for ruptured IAs}

\subsubsection{Outcome assessment}

Results of management outcomes in patients with SAH from a ruptured IA have not been reported in a standardized manner. In population based studies, outcome is often measured by the cumulative case-fatality rate at one to six months after SAH $[131,290,301,369,381]$. The case-fatality rate allows observation of trends over long periods of time but it does not take into account the functional state of the patient. Most studies evaluating treatment of SAH use either the Glasgow outcome score (GOS) [152], or the Rankin scale [322], both of which divide patients into categories based on their functional capacity.

\subsubsection{Treatment outcome}

Although the case-fatality rates of SAH have been slightly declining over the last three decades [131], they are still as high as $35-50 \%$ $[84,301,381]$. The outcome depends strongly on the admission policy of the hospital and especially on the proportion of poor-grade patients. Many large surgical series from referral centers are strongly biased towards patients in better preoperative condition [430]. In centers with active admission policy and little selection bias so that even poor-grade patients are treated, $60-80 \%$ of patients had a favorable outcome (GOS $\geq 4)[123,193,350,356]$. It is even more difficult to compare outcome of surgical treatment in between different patient series as there are many factors causing selection bias. These include clinical condition before treatment, aneurysm location, timing of treatment, methods for outcome evaluation, length of follow-up, and prospective vs. retrospective nature of the data.

\subsubsection{Predictors for outcome after $S A H$}

Factors which are generally recognized to predict outcome after SAH are: neurological grade on admission, age, amount of blood on 
the preoperative CT scan, intracerebral hematoma (ICH), intraventricular hemorrhage (IVH), and aneurysm location [339]. The neurological grade on admission has the strongest effect on outcome [260,287,329,342]. In patients with initial clinical grade IV or V, a favorable result was seen in only $30-50 \%$ of the cases irrespective of the treatment method [28,173,204,249,426], whereas good preoperative clinical grade (Grade I or II) predicted a favorable result in $80-90 \%$ of the patients $[123,173,287,350]$. The second most important factor is the age [339]. Younger patients seem to be more likely to tolerate systemic stress caused by acute SAH and, therefore, recover better than the elderly $[207,287,329,342]$. Thick blood clots in basal cisterns (Fisher grade $\geq 3$ ), a risk factor for development of delayed vasospasm [80], also predict a less favorable outcome [110]. Neurological grade, age and blood on CT scan seem to be more important than other factors in predicting the outcome after SAH [339].

\subsubsection{Long-term follow-up after SAH}

\subsubsection{De novo aneurysms and rebleeding}

The risk of rebleeding from a treated aneurysm is of major concern for a patient after microsurgical or endovascular treatment. Multiple aneurysms, usually present already at the first SAH or rarely developing later (de novo) [332], are detected in about one third of $\mathrm{SAH}$ patients [331]. They are considered to be a predisposing factor for recurrent $\mathrm{SAH}$ together with smoking and hypertension [428]. The cumulative rupture rate increases with follow-up and the relative risk compared to the general population is higher [160,419].

De novo aneurysms developed with the annual rate of $0.84 \%$ in a previous Finnish study with a median follow-up time of 19 years [161]. In a Japanese study, the annual rate of de novo aneurysm formation was $0.89 \%$, being much higher than the $0.26 \%$ rate for re-growth of completely clipped aneurysm [408]. The
International Study of Unruptured Intracranial Aneurysms (ISUIA) suggested a 5-year cumulative rupture rate of $1.5 \%$ for unruptured anterior circulation aneurysms in patients with previous SAH [431]. A recent study from The Netherlands states the incidence of recurrent SAH after clipping of ruptured aneurysms in a 10-year follow-up as $3.2 \%$ with $77 \%$ due to de novo aneurysms [427]. Unfortunately, there are no population based studies on SAH with median follow-up times of over 15-20 years. The present belief is that patients with multiple aneurysms and a history of SAH are at increased risk of developing new aneurysms in the long run $[157,159]$, but these aneurysms are likely to have the same rupture risk as other unruptured aneurysms.

\subsubsection{Long-term mortality}

Relatively little is known about the longterm survival after aneurysmal SAH. Most studies report long-term outcome as early as 6 or 12 months after $\mathrm{SAH}[7,105,131,338]$. There are only two population based SAH studies with a median follow-up of over 5 years. Ronkainen et al. showed that Finnish SAH patients with good recovery at 12 months and successful treatment of their ruptured aneurysm had mortality rate twice as high as the general population during a median follow-up of 7.5 years [337]. Olaffson's series of 44 Icelandic patients who survived over 6 months after SAH showed that the patients who had severe disability at 6 months experienced excess mortality during the first 10 years of follow-up [281]. Both of these studies included all SAH patients irrespective of the location of the aneurysm.

\subsubsection{Management of unruptured IAs}

In the management of patients with unruptured IAs the risk of treatment has to be weighed against the risk of rupture and subsequent complications. Annual rupture rate for unruptured aneurysms has been estimated to 
be about $1 \%[160,161,328,448]$. Risk factors for aneurysm rupture include the female gender, smoking, older age, high blood pressure, aneurysm size, aneurysm location, and country of origin $[76,155,159,160,218,317,328]$. Of these, smoking seems to have the highest attributable risk of almost $50 \%$ and even if smoking is stopped, the risk of SAH remains higher than in nonsmokers $[76,189]$. The largest study on unruptured aneurysms with 4060 patients and mean follow-up of four years, the ISUIA study, identified in the multivariate analysis of their prospective cohort only the aneurysm size and location as predictors for rupture, although there was some selection bias of aneurysm location between the conservatively and actively treated groups [431]. In a meta-analysis of surgical series from 1970 to 1996, the mortality and morbidity rates related to treatment of unruptured IAs were $2.6 \%$ and $11 \%$, respectively [317], but the giant and posterior circulation aneurysms were overrepresented in this analysis so that for most aneurysms the risk is probably lower [154]. The ISUIA study reported $1.5-2.3 \%$ mortality and $10-12 \%$ morbidity in a prospective follow-up [431]. It seems that an appropriate risk/benefit analysis requires thorough knowledge of the treatment results of the particular center or the physician giving the treatment. Unlike in ruptured aneurysms, where the initial impact of SAH strongly determines the management outcome, in unruptured aneurysms it is the experience, knowledge and skills of the treating physician and his team that have the main impact on the outcome [194]. In Finland, with higher rupture risk, even small, unruptured aneurysms are treated actively, microneurosurgery being often preferred over endovascular treatment due to the high proportion of middle cerebral artery (MCA) aneurysms (40\%) [49], and the better long-term results associated with clipping [251].

\subsection{History of intracranial aneurysm treatment}

\subsubsection{Before microneurosurgery}

\subsubsection{Intracranial aneurysms in historical context}

Found in the Ebers Papyrus and attributed to Imhotep (2725 BC), the first record of an arterial aneurysm described the treatment of a bulging aneurysm with a fire-glazed instrument by an Egyptian physician [219]. In 117 BC, Flaenius Rufus, a physician from Ephesus and trained in Alexandria, made a notion that arterial dilatation could be caused by trauma [51], but it was the Greek physician Galen of Pergamum who first properly defined and described the entity of an arterial aneurysm in general in 200 AD [225]. During the next 1500 years, Islamic physicians expanded their knowledge on aneurysms, their origins and sites, whereas in the western cultures studies on human anatomy were largely stagnant for both religious and cultural reasons $[168,169]$. The modern definition of aneurysm as a dilatation of a weakened artery was made by Lancisis in 1728 [429]. Intracranial aneurysms were definitely described for the first time in the autopsy reports by Morgagni (1761, Padua), Biumi (1765, Milan), and Blane (1800, London) $[22,27,256]$. At that time there were no reports on their treatment as the IAs were usually found only at post mortem examinations. It was not until Heinrich Quincke introduced the lumbar puncture in 1891 [314] that the diagnosis of $\mathrm{SAH}$ became truly possible in living patients, a development which in turn led to substantial discussions about the possibilities of treating patients with aneurysmal SAH.

\subsubsection{Hunterian ligation}

In 1805, Cooper performed carotid ligation for an extracranial carotid artery aneurysm with a fatal result [39]. Undeterred, he performed the same procedure three years later, this time suc- 
cessfully $[38,40]$. Arterial ligation in general was popularized in the 18th century by John Hunter who demonstrated a safe and reproducible means of proximal femoral artery ligation for popliteal aneurysms as an alternative to leg amputation [136]. Named in his honor, Hunterian ligation of the internal carotid artery (ICA) was adopted by many surgeons as a method for treating intracranial vascular pathologies. In 1809, Benjamin Travers was the first to report a successful treatment of an intracranial lesion (carotid cavernous fistula) by this method [403], but it was much later before the Hunterian ligation was used to treat an actual IA.

\subsubsection{Carotid occlusion}

In 1885 , in London, Sir Victor Horsley operated on a 48-year old woman thought to suffer from a tumor in the middle cranial fossa. Intraoperative finding was a pulsating mass, most likely an aneurysm, which forced Horsley to change his surgical strategy. Instead of removing the lesion, he ligated the right common carotid artery. The patient was reported to be doing well five years later [180]. Many surgeons after Horsley ligated the ICA on encountering an IA during an intracranial operation. However, these ligations were quite frequently followed by cerebral infarctions, which led to the need of differentiation of patients who would tolerate occlusion. Matas developed a preoperative compression test for this purpose in 1911 [236], but it was not until 1924 that the first planned ICA ligation for IA, diagnosed preoperatively, was carried out by Trotter on a patient with traumatic aneurysm causing severe epistaxis [354]. A variety of more sophisticated techniques were developed over the following years to allow gradual occlusion of the carotid artery while building up the collateral circulation including Matas's band from aluminum strips, double fascia band, and Neff's clamp $[235,263,297,307]$. Later on came the Dott, Crutchfield, Selverstone, and Kindt clamps, some of which remained in use until the late 1970s when they were outdated first by microneurosurgery and later by endovascular surgery $[42,63,95,358]$. Even with gradual occlusion of the carotid artery by these different clamps, the mortality rates were around $20 \%$ and the stroke rate was as high as $30 \%[268,306,354]$.

\subsubsection{Cerebral angiography}

The introduction of cerebral angiography by António Egas Moniz in 1927 not only revolutionized the diagnostics of cerebral aneurysms but also played a key role in starting the development of IA treatment [253]. Before that, plain $\mathrm{x}$-rays, pneumoencephalography, and myelography were the basic imaging methods of the central nervous system. In this way only some calcified aneurysms could be seen and even those were initially often mistaken for tumors such as calcified meningeomas. By 1931, Moniz was able to perform a complete carotid angiogram including arterial and venous phases, and two years later, he published an article on IA diagnostics by means of angiography [252]. In the same year, Dott was the first to operate on an aneurysm previously diagnosed by angiography [62]. Initially, one of the problems associated with angiography was the radioactivity of Thorotrast ${ }^{\circledR}$, the contrast medium used at that time, which remained in the liver and in fact proved to be carcinogenic [303]. This contrast agent was exchanged for a 35\% Diodrast ${ }^{\circledR}$ solution, the intravenous pyelogram contrast medium, which, turning out to be much safer, became widely accepted [305,451], though still far from being comparable to the modern contrast media [58]. Although Moniz had opacified even the posterior circulation by an open retrograde subclavian injection, it was Krayenbühl in 1941 who first demonstrated an aneurysm on the vertebro-basilar system using the same method [351]. Angiography's usefulness as a diagnostic tool increased even further with the percutaneous carotid puncture technique described by Lohman and Myerson in 1936 and Shimidzu in 1937 [221,402], and later with 
Seldinger's technique of catheter angiography through the percutaneous transfemoral route published in 1953 [357].

\subsubsection{Wrapping and trapping}

Norman McComish Dott of Edinburgh (a pupil of Cushing's) was the first to be credited with direct attack on a ruptured aneurysm in 1931. Without angiographic assistance, he performed a frontal craniotomy on a 53-year old patient, who was the financial director of Dott's hospital with three previous bleeds and a III nerve palsy. Intraoperatively, a 3-mm aneurysm in the region of ICA bifurcation was wrapped with muscle harvested from the patient's thigh, and the patient made a good recovery [62]. Additional reports by Tönnis, Dandy, and Jefferson added to the literature on wrapping $[44,151,398]$. Herbert Olivecrona, founder of Scandinavian neurosurgery, was the first to effectively treat a posterior circulation aneurysm in Stockholm in 1932. During operation on what he initially thought to be a posterior fossa tumor, he found a large, thrombosed posterior inferior cerebellar artery (PICA) aneurysm, which he trapped and excised [270]. The patient was reported to be doing well 17 years later [220]. In 1936, Dandy invented a new technique to treat an ICA aneurysm in or near the cavernous sinus by ligating ICA both intracranially and extracranially, thus trapping the aneurysm [45].

\subsubsection{Clipping}

A major revolution in the treatment of IAs came with the invention of metallic clips [302]. In 1911, in his quest to develop tools for tumor resections, Harvey Cushing produced what was to become known as "the silver clip" or "Cushing clip" [43]. Cushing used this clip in tumor surgeries for "placement on inaccessible vessels, which, though within reach of a clamp, are either too delicate or in a position too awkward for safe ligation" [43]. The original silver clip, made out of round silver wire, was first modi- fied in 1927 by McKenzie into a V-shaped clip using flat wire [243], and later in 1949 by Duane into a U-shaped clip [66]. Cushing never used his invention for intracranial aneurysm surgery, instead, it was his competitor Walter Dandy who clipped the first aneurysm on March $23^{\text {rd }} 1937$ [46]. He exposed a saccular posterior communicating artery (PCoA) aneurysm causing oculomotor palsy, and clipped the aneurysm at the neck with a Cushing-McKenzie type silver clip. The oculomotor palsy subsided six weeks later [46]. This new clipping method allowed neurosurgeons to exclude an aneurysm selectively from the intracranial circulation, a concept that marked the beginning of the modern era of aneurysm surgery.

\subsubsection{Aneurysm clip development}

The clip used by Dandy in 1937 evolved significantly over the next decades. First there was the development of an adjustable clip which could be re-opened and repositioned, a winged clip with a special applicator modified by Olivecrona [271]. The mechanism of crossing the legs of a spring forceps was invented already in 1840 by the French medical instrument maker Joseph Charrière [242], and the idea became the basis for even the modern-day clips. Schwartz introduced a miniature spring forceps clip to allow re-opening of the clip to prevent shearing and tearing of the aneurysm base [238]. As Schwartz's clip was robust and the applicator was awkward to enable effective use intracranially this led Mayfield and Kees to modify the cross-legged clip in 1952 into a tool designed specifically for aneurysm surgery [238]. The Mayfield clips were produced with different lengths and angulations, and gained wide popularity among neurosurgeons practicing aneurysm surgery [238]. Over the following decades, substantial modifications were made as various neurosurgeons suggested improvements [224]. McFadden suggested round instead of flat blades [238], while Sundt and Nofzinger developed a Teflon-lined, ves- 
sel encircling clip-graft in 1967 [389], and in 1969 Kees made the first fenestrated clips for Drake's needs [64]. Scoville produced a miniature torsion-bar aneurysm clip in 1966 [355], and Heifetz designed a clip with an internal wire spring in 1968 [115]. Interestingly, the original idea behind the Heifetz clip came from a Finnish neurosurgeon, Stig Nyström, who invented a silver aneurysm clip with an internal spring in 1959 (S Nyström, personal communication) [275]. Nyström and a few others used these clips which, however, never gained widespread popularity since obtaining a patent for this kind of product was very difficult at that time in Finland (S Nyström, personal communication). The Heifetz clip was similar in design to the Nyström clip, though somewhat more delicate, but the biggest difference was that it was made from steel. The clip design with the longest life cycle so far originated from the collaboration between McFaden and Kees in 1970 [241]. This design implemented a mechanism to prevent scissoring, and an additional spring loop to increase the closing force while allowing the whole clip to be made from the same material [240]. Later, other neurosurgeons such as Yaşargil, Sugita, Drake, Perneczky, and Spetzler have been intensively involved with modifying the aneurysm clips to better suit the specific needs of microneurosurgery $[213,214,296,302$, $387,447]$. It is important to note that especially in the 1950 s and 1960s, before the Mayfield clips came to wide use, many aneurysms were tied at the neck with linen or silk thread [23].

\subsubsection{Microneurosurgery}

\subsubsection{Operating microscope}

In the latter half of the 19th century, microscopes were already used in industry and scientific research, but in clinical surgery their use was preceded by loupe magnification. True compound magnification was used for the first time in surgery by the German physician Saemisch, who wore loupes in 1876 [363]. In
1921, the Swedish otolaryngologist Carl Nylen, inspired by a paper of Maier and Lion on observations of endolymph movements in the ears of live pigeons using a dissecting microscope, conceived, built and used the world's first surgical monocular microscope [59,274]. This invention was followed the next year by his chief Gunnar Holmgren, who attached an external light source to an existing Zeiss dissecting microscope, thus introducing the first binocular surgical microscope [59]. The original surgical microscopes were rather robust, had a limited field of vision, lacked stable and freely movable support, and had an insufficient coaxial light source [325]. In the early 1950s, several technical advancements encouraged microscopes to be used more frequently. Hans Littman of Zeiss Company developed the optical design for changing magnification without changing the focal length and he designed the first series-produced operating microscope, Zeiss OpMi 1 (Zeiss Operating Microscope Number One) in 1953 [128,228]. Later in 1960, it was again Littman who designed the first two-person series-produced operating microscope, the diploscope, for the microsurgical laboratory in Burlington, Vermont [200].

\subsubsection{Development of microneurosurgery}

On August $1^{\text {st }}, 1957$, Theodore Kurze, at the University of Southern California in Los Angeles, was the first neurosurgeon to use a microscope in the operating room $[61,200]$. A year later, Raymond Madiford Peardon Donaghy established the world's first microsurgery research and training laboratory in Burlington, Vermont $[61,200,239,425]$. He was interested in treating cortical strokes by removing clots from inside the thrombosed artery and repairing it afterwards. He collaborated with a vascular surgeon, Julius Jacobson, first using the Zeiss OpMi 1 borrowed from the ENT services [29,61]. Later, with the help of the diploscope designed by Littman and special sets of instruments developed for microsurgery, Jacobson and Suarez 
successfully anastomosed carotid arteries in dogs and rabbits, and published their findings on the power of microscope in small-vessel anastomoses in $1960[144,145,385]$. The same year, Donaghy used the operating microscope to perform the first embolectomy and endarterectomy on the middle cerebral artery [145]. In 1962, a cardiac surgeon in Zürich, Åke Senning, asked neurosurgeon Hugo Krayenbühl to remove an embolus from the MCA in a young patient with hemiplegia after cardiac surgery. At that time Krayenbühl did not think this possible, but the idea of surgery on small intracranial vessels remained and in 1965 he dispatched his pupil, M. Gazi Yaşargil, to the United States to learn the new art of microsurgery [6o]. Yaşargil originally approached Jacobsen, who referred him to Donaghy's laboratory in Vermont, where he spent the next year mastering microsurgical techniques under the guidance of Miss Esther Roberts by means of performing anastomoses of the superficial temporal artery (STA) to the middle cerebral artery (STA-MCA bypass) in dogs $[60,61]$. Upon returning back to Zürich, Yaşargil performed the first STA-MCA bypass on a human patient on October $30^{\text {th }}, 1967$ [60]. Less than 24 hours later, Donaghy performed the same operation in Burlington, and both of these surgeries were successful [61]. Yaşargil's return to Zürich and the first microsurgical operation on February $1^{\text {st }} 1967$ marked the beginning of a new era in microneurosurgery. Yaşargil's devotion to development of operative approaches, techniques and instrumentation has been appraised by many of his colleagues [82,394]. In 1979, Donaghy wrote: "Little was it realized at this time (in 1965), even by Hugo Krayenbühl, that this young Turk was destined to do more for the development of microneurosurgery in the human nervous system than any other man" [61].

\subsubsection{Other technical developments}

Microneurosurgery was very much dependent on technical and anesthesiological in- novations. On the microscope front, Yaşargil's collaboration with the Contraves Company resulted in designing a counterbalanced stand for microscope, originally suggested by Malis, with a system of electromagnetic brakes permitting full mobility and perfect stability $[230,447]$. The major advantage of microscopes, a clear, bright, 3D magnified vision, required a bloodless operating field. This could be obtained with the bipolar coagulator, first described by Greenwood in 1940, and subsequently improved by Malis $[100,101,228-230,239]$. Compared to unipolar coagulation, in which the current spreads over a larger area, bipolar coagulation allows to limit the coagulation to precisely targeted structures. In addition, the bipolar forceps could serve as a general dissection instrument [230,447]. Microsurgical instrumentation has evolved gradually from the early days of microsurgery to reach the highly sophisticated level of the present time. But even the best technical advances would not have sufficed without the developments in balanced neuroanesthesia with constant monitoring of different physiological parameters [321]. The introduction of controlled hyperventilation and effective osmotic agents, first urea in 1954 by Javid [148-150], and later mannitol in 1961 by Wise and Chater $[364,436,437]$, provided additional space for intracranial procedures and diminished the grave dangers of opening the dura in the presence of a tight brain. These achievements also paved the road for the concept of early surgery in ruptured aneurysms.

\subsubsection{Microneurosurgery applied to intracranial aneurysms}

Microsurgery started expanding into aneurysm surgery in the 1960s. Kurze was the first to use the microscope systematically for all his aneurysm cases starting in 1958, but he never published or presented his series [200]. Adams and Witt started using an ENT microscope for aneurysms in 1963 and presented their experience at the meeting of the Neurosurgical 
Society of America in 1964 [3]. The first published series on aneurysm surgery using a microscope came from Pool and Colton in 1966, who published their experience in 13 patients [304]. The next year Rand and Janetta reported a basilar bifurcation and a PICA aneurysm, both ligated with silk thread under the microscope [320]. They emphasized the power of microscope in distinguishing the small perforators in basilar bifurcation aneurysms and compared their experience with Drake's, who at that time had a $50 \%$ mortality in his series of eight basilar bifurcation aneurysms operated without the microscope but with loupes [65]. Microneurosurgery was spreading fast during this period as an increasing number of neurosurgeons realized the advantages of microsurgery on their results. Publications by Lougheed (1969), Cophignon (1973), Hollin (1973), and Guidetti (1973) totaled 236 anterior circulation aneurysms operated on under the microscope $[41,104,130,223]$. The real groundbreaking series was that by Krayenbühl and Yaşargil in 1972, describing Yaşargil's results over a fouryear period from 1967 to 1971 , in which total mortality was only 4\% in 231 patients with microsurgically operated anterior circulation aneurysms [199]. With further experience and development of instrumentation mortality in Yaşargil's series dropped to $2 \%$ in the time period from 1970 to 1974 , setting a new standard for aneurysm surgery (late surgery) [446]. Meanwhile Drake, already using a microscope (since 1970), pioneered surgery for posterior circulation aneurysms [64].

\subsubsection{Endovascular surgery}

\subsubsection{Balloon occlusion}

The first attempts to cure brain aneurysms from the endovascular side using injection of either hog or horse hair into the aneurysm sac during open surgery were reported by Gallagher in 1964 [94]. His idea was that the shingles on the end of the hair, placed inside the aneurysm, would create a mechanical nidus for clotting, yet complete thrombosis of the aneurysm occurred in only nine of the 15 patients [94]. In 1971, Serbinenko, a Russian neurosurgeon from the Burdenko Institute in Moscow, reported the use of inflatable balloons for temporary occlusion of intracranial vessels and carotid cavernous fistulae [361]. By 1974, he reported the use of selective catheterization to deliver and deploy detachable balloons filled with a hardening agent (liquid silicone) for the treatment of various intracranial vascular lesions, including aneurysms, in more than 300 patients [360]. Although initially a promising method, significant complications and recanalization of aneurysms were later reported even when using other material to fill the balloon $[57,126,127,254,333]$. A big step in endovascular therapy came with the introduction of microcatheters and microguidewires by Target Therapeutics (Fremont, CA, USA) in 1986, which allowed safer and more effective exploration of intracranial vessels [182].

\subsubsection{Coiling}

The most important technical development in endovascular surgery for the treatment of IAs was the invention of Guglielmi detachable coils (GDC) in 1990 [102,103]. The initial coils for endovascular use were available as free coils, i.e. they had to be pushed through the microcatheter with a special wire referred to as the coil pusher, and once they left the tip of the catheter they could not be pulled back [326]. Guglielmi, together with Sepetka, developed the first generation of electrolytically detachable platinum coils allowing proper positioning inside the aneurysm before the release [103]. In 1991, Guglielmi published the first series of 15 patients treated with this new method [102]. The method spread fast and several large series on the use of GDCs in aneurysm treatment were published in the following years $[36,424]$. Owing to the development of new techniques, such as balloon remodeling technique intro- 
duced by Moret in 1997 [255], and introduction of new coils and other embolic material, an increasing number of aneurysms could be treated by endovascular methods [309]. This also started a competition between microneurosurgery and endovascular surgery forcing supporters of either technique to seek faster, safer, less invasive, and more durable techniques of IA treatment for the benefit of the patients [121].

\subsubsection{Aneurysm surgery in Finland}

\subsubsection{Aarno Snellman, founder of Finnish neurosurgery}

The first neurosurgical operations in Finland were performed in the beginning of the 2oth century by surgeons such as Schultén, Krogius, Faltin, Palmén, Kalima and Seiro, but it is Aarno Snellman who is considered the founder of neurosurgery in Finland [400]. The Finnish Red Cross Hospital, which was the only center for Finnish neurosurgery until 1967, was founded in 1932 by Marshall Mannerheim as a trauma hospital [401]. Already during the first years, the number of patients with different head injuries was so significant that an evident need for a trained neurosurgeon and specialized nurses was soon identified. In 1935, Professor of Surgery Simo A. Brofeldt sent his younger colleague, 42year old Aarno Snellman, to visit Olivecrona in Stockholm [401]. Snellman spent there half a year, closely observing Olivecrona's work. Upon his return, he performed the first neurosurgical operation on September $18^{\text {th }}, 1935$ [401]. This event is generally considered as the true beginning of neurosurgery in Finland.

\subsubsection{Angiography in Finland}

The initially relatively poor surgical results were mainly due to insufficient preoperative diagnostics. Realizing the importance of preoperative imaging Snellman convinced his colleague from radiology, Yrjö Lassila, to visit Erik Lysholm in Stockholm [400]. The first cerebral angiographies were performed after Lassila's return to Helsinki in 1936 [375]. At that time, angiography was often performed only on one side as it required surgical exposure of the carotid artery at the neck and four to six staff members to perform the relatively lengthy procedure: one to hold the needle, one to inject the contrast agent, one to use the X-ray tube, one to change the films, one to hold the patient's head, and one to show light. The procedure was quite risky for the patient, and there was one death among the first 44 cases ( $2 \%$ mortality) [375]. There were also some quite unexpected complications such as the situation where the surgeon injecting the contrast agent got an electric shock from the X-ray tube, fell unconscious to the floor, and while falling, accidentally pulled the loop of the silk thread passed under the patient's carotid artery, thus causing total transection of this artery. Fortunately, the assistant was able to save the situation and, as Snellman stated in his report, "no one was left with any permanent consequences from this dramatic situation" [375]. Before 1948, the number of cerebral angiographies was only 1520 per year [402], but with the introduction of percutaneous technique at the end of 1948, the number of angiographies started gradually to rise with more than 170 cerebral angiographies performed in 1949 [402].

\subsubsection{World War II and the late $1940 \mathrm{~s}$}

World War II had a significant effect on the development of neurosurgery in Finland. On the one hand, the war effort diminished the possibilities to treat the civilian population, on the other hand the high number of head injuries boosted the development of the neurosurgical treatment of head trauma [401]. During this period, several neurosurgeons from other Scandinavian countries worked as volunteers in Finland helping with the high casualty load. Among others there were Lars Leksell, Nils Lundberg and Olof Sjöqvist from Sweden, and Eduard Busch from Denmark [400]. After the 
war, it became evident that neurosurgery was needed as a separate specialty. Aarno Snellman was appointed Professor of Neurosurgery at the Helsinki University in 1947, and in the same year, the medical students had their first, planned course in neurosurgery [374]. The next year, Teuvo Mäkelä, who worked in neurosurgery since 1940 in charge of head injury patients, was appointed First Assistant Professor of Neurosurgery [400]. An important administrative change came in 1946, when the Finnish government decided that the state would pay the expenses of neurosurgical treatment [401]. With this decision neurosurgical treatment became, at least in theory, available for the whole Finnish population. The limiting factors were hospital resources (there was initially only one ward available) and the relatively long distances within the country. This explains partly why, especially in the early years, e.g. aneurysm patients sought operative treatment several months after the initial rupture, and only those in good condition were selected. Neurosurgery remained centralized in Helsinki until 1967, when the department of neurosurgery in Turku was founded, to be later followed by neurosurgical departments in Kuopio (1977), Oulu (1977) and Tampere (1983) [406].

\subsubsection{Initial steps in aneurysm surgery}

Aneurysm surgery in Finland started relatively slowly. In 1939, from among the first 44 patients undergoing diagnostic angiography at the Neurosurgery Department in Helsinki, only nine were diagnosed with an IA [375]. It is difficult to establish the exact date of the first aneurysm surgery in Finland. In their paper on 52 MCA aneurysms from 1958, af Björkesten and Troupp mention a patient who was operated on in 1937, and who subsequently died from wound infection [25]. Unfortunately, they neither specified the exact date nor the surgical procedure used. During World War II, the main focus of Finnish neurosurgery was, as stated before, on traumatology. Later, the better avail-

Table 1. The early publications from Helsinki on the treatment of intracranial aneurysms.

\begin{tabular}{|c|c|c|c|}
\hline Year & Author(s) & Journal & Publication \\
\hline 1957 & $\begin{array}{l}\text { af Björkesten and } \\
\text { Troupp [26] }\end{array}$ & J Neurosurg & $\begin{array}{l}\text { Prognosis of subarachnoid hemorrhage, a comparison between pa- } \\
\text { tients with verified aneurysms and patients with normal angiograms }\end{array}$ \\
\hline 1958 & $\begin{array}{l}\text { af Björkesten and } \\
\text { Troupp [25] }\end{array}$ & Acta Chir Scand & Aneurysms of the middle cerebral artery, a report on 52 cases \\
\hline 1958 & af Björkesten [23] & J Neurosurg & $\begin{array}{l}\text { Arterial aneurysms of the internal carotid artery and its bifurcation, } \\
\text { an analysis of } 69 \text { aneurysms }\end{array}$ \\
\hline 1959 & $\begin{array}{l}\text { Snellman, Mäkelä } \\
\text { and Nyström [373] }\end{array}$ & Neuro-Chirurgie & $\begin{array}{l}\text { Considerations on the aneurysms of the anterior communicating } \\
\text { artery, a report on } 52 \text { cases [in French] }\end{array}$ \\
\hline 1960 & $\begin{array}{l}\text { Laitinen and } \\
\text { Snellman [205] }\end{array}$ & J Neurosurg & Aneurysms of the pericallosal artery, a study of 14 cases \\
\hline 1960 & $\begin{array}{l}\text { af Björkesten and } \\
\text { Troupp [24] }\end{array}$ & Acta Chir Scand & Multiple intracranial arterial aneurysms \\
\hline 1961 & $\begin{array}{l}\text { Laitinen and } \\
\text { Troupp [206] }\end{array}$ & Acta Neurol Scand & $\begin{array}{l}\text { Reliability of partial ligation in the aneurysm treatment of intracra- } \\
\text { nial arterial aneurysm }\end{array}$ \\
\hline 1962 & Heiskanen [117] & Acta Neurol Scand & Large intracranial aneurysms \\
\hline 1964 & $\begin{array}{l}\text { Troupp and } \\
\text { Laitinen [407] }\end{array}$ & Acta Neurol Scand & $\begin{array}{l}\text { Reliability of partial ligation in the aneurysm treatment of intracranial } \\
\text { arterial aneurysm. II }\end{array}$ \\
\hline 1971 & $\begin{array}{l}\text { Troupp and af } \\
\text { Björkesten [405] }\end{array}$ & J Neurosurg & $\begin{array}{l}\text { Results of controlled trial of late surgical versus conservative treatment } \\
\text { of intracranial arterial aneurysms }\end{array}$ \\
\hline
\end{tabular}


ability of angiographic contrast medium, introduction of percutaneous puncture technique, and routine use of bilateral carotid angiography built the basis for wider diagnosing of IAs. Bilateral angiography was seldom performed before 1948 [26], and routine postoperative angiography controls were started in 1952 [206]. According to af Björkesten, the direct intracranial attack became the method of choice for treating IAs in 1952 [23]. Between 1936 and 1958, 320 patients with angiographically and/ or intraoperatively verified IAs were treated at the Neurosurgery Department in Helsinki [2325,205]. Of these, 291 (91\%) were diagnosed after 1951 [206]. From the hospital's operative records started in 1951 (the earlier volumes have unfortunately been lost or destroyed) we can see that although during the first year (1951) there were only three surgeries for IAs, the numbers started to rise steadily, with eight surgeries in 1952, 12 in 1953, 29 in 1954, and 30 in 1955. Most of these operations were performed by Snellman, Mäkelä and af Björkesten. The aneurysms were usually occluded directly with either linen thread ligature $(42 \%)$ or silver clips (39\%) (spring clips were not introduced until the 1960s) [206]. The remaining aneurysms were wrapped, trapped, treated with ICA or proximal ACA occlusion, or, infrequently, only explored. Surgical mortality between 1936 to 1958 was $9 \%$ [206]. From the very start of aneurysm surgery in Finland, the results were actively reported in international journals (Table 1), and this practice has continued ever since.

\subsubsection{Microneurosurgery and endovascular surgery}

The first neurosurgeon to use the operating microscope in Finland was Tapio Törmä in Turku in the beginning of the 1970s (personal communication J Hernesniemi). The first operating microscope at the Neurosurgery Department in Helsinki was acquired in 1974, after a delay of one year due the university financial services who considered the item a very expensive and unnecessary piece of equipment [406]. Initially, the microscope was used by neurosurgeons operating on aneurysms, small meningeomas, and acoustic schwannomas. Since laboratory training in microsurgical techniques was not deemed necessary, they usually started the use directly in the OR. A Turkish born neurosurgeon Davut Tovi from Umeå held a laboratory course in Helsinki in January 1975, during which he also demonstrated the use of microscope in the OR while the intraoperative scene was being displayed on a TV monitor (personal communication J. Hernesniemi). Interestingly, during the first years of microneurosurgery on aneurysms, intraoperative rupture often compelled the neurosurgeon to abandon the microscope and move back to macrosurgery so that he could "see better" the rupture site. But the younger generation was already starting with microsurgical laboratory training; among them Juha Hernesniemi who after his first aneurysm surgery in 1976 has performed more than 3150 aneurysm operations, all of them using a microscope. A year after visiting Yaşargil in Zürich in 1982, Hernesniemi became the first neurosurgeon in Finland to use a counterbalanced microscope equipped with a mouth switch (personal communication J. Hernesniemi). Surgery on unruptured aneurysms in patients with previous SAH started in 1979 [116], and the first paper on aneurysm surgery in patients with only incidental, unruptured aneurysms was published in 1987 [118]. Endovascular treatment of IAs began in 1991 (personal communication M. Porras). 


\subsection{Microsurgical anatomy for DACA aneurysms}

\subsubsection{Anterior cerebral artery}

The ACA, the smaller of the two terminal branches of the ICA, arises at the medial end of the Sylvian fissure lateral to the optic chiasm. It traverses the optic chiasm or the optic nerve, and ascends in front of the lamina terminalis in the lamina terminalis cistern. Before entering the interhemispheric fissure, it is connected to the opposite ACA via the anterior communicating artery ( $A C O A)$ (Fig. 1). Inside the interhemispheric fissure, the left and right ACA trunks run parallel along the corpus callosum, in the pericallosal cistern. Both trunks give origin to several cortical, subcortical, and callosal branches, some of which may cross over to the opposite side. Cortical branches of the ACA supply the anterior two thirds of the medial aspects of the cerebral hemisphere as well as the superior portions of the superior frontal, precentral, and postcentral gyri [294].

The ACA is divided into five segments ( $A_{1-}$ A5) according to Fischer (Fig. 1)[79]. The $A_{1}$ segment is located between the ICA bifurcation and the $A C O A$. The $A_{2}$ segment extends from the $A C O A$ to the region between the rostrum and the genu of the corpus callosum (GCC). The A3 segment curves around the GCC and ends at the rostral part of the body of the corpus callosum. The $\mathrm{A}_{4}$ and $\mathrm{A}_{5}$ segments follow the superior surface of the corpus callosum with a virtual plane of division at the level of the coronary suture. Traditionally, the ACA has been divided into a proximal part $\left(A_{1}\right)$ and a distal part ( $A_{2}$ to $\left.A 5\right)$, the latter also called the pericallosal artery $[97,162,178,294,417,445]$.

\subsubsection{A1 segment of $A C A$}

The $A_{1}$ segment arises from the ICA in the carotid cistern, and with a medial and somewhat anterior course it enters the cistern of the

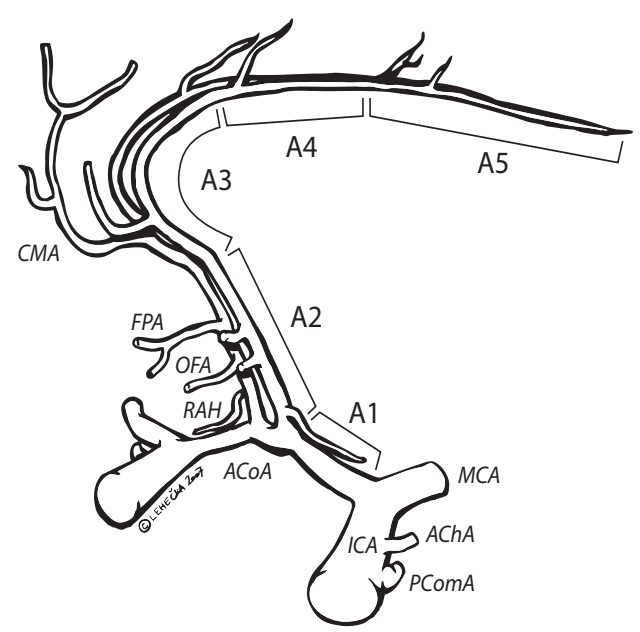

Fig. 1. The segments ( $A_{1}$ to $\left.A_{5}\right)$ and branches of the anterior cerebral artery (ACA).

lamina terminalis. At this point, the A1 segment is encased by thick arachnoid bands that extend from the olfactory triangle to the lateral side of the optic nerve. When mobilizing the $A_{1}$ segment, these bands require careful dissection, especially when toughened by previous SAHs. The course of the $A_{1}$ segment varies in length and dominance, and it may loop under the frontal lobe [340,442]. Importantly, one $\mathrm{A}_{1}$ is dominant over the other in the majority of cases [442]. The A1s join the ACoA complex mostly above the chiasm (70\%), less frequently above the optic nerves (30\%) [324].

\subsubsection{Anterior communicating artery ( $A C O A)$ complex}

The ACoA is the fundamental anastomotic part of the anterior circle of Willis. Detailed microneurosurgical anatomy of the ACoA complex and its anatomic variations are reported by Yaşargil [442] and others [11,67,78,97,166,232, $295,324,327,362,392,412]$. The location and orientation of the ACoA complex varies highly with respect to the optic chiasm and the anterior skull base, depending on the diameters, lengths, and courses of the A1s [295,324,442]. In 30 brains studied by Serizawa et al., two (6\%) 
ACoAs were high above the optic chiasm [362]. The length, shape, and diameter of the ACoA also vary $[295,324,442]$. The average diameters of ACoAs and A1s were $1.6 \mathrm{~mm}$ and $2.6 \mathrm{~mm}$, respectively, in a series of 50 cadavers, and the average length of the ACoA was between 2 and 3 $\mathrm{mm}$ (range 0.3-7.0 mm) [295]. In cross sections, ACoAs can be round, triangular, or even almost flat. The diameter of the $A_{1}-A_{2}$ junctions was of the same size in $74 \%$, larger in the right side in $14 \%$, and larger in the left side in $12 \%$ [295].

\subsubsection{Perforating branches of $A C O A$}

It is difficult to overestimate the importance of the perforators of the ACoA complex region. The complex microneurosurgical anatomy of the perforating branches arising from the $A C O A$ complex has been described in detail $[11,12,67$, 198,232,295,324,362,392,415,423,442]. Their supply areas, laterality, pattern of origin, and number vary a great deal $[67,119,232,295,362$, $415,423,442]$. Most of them originate, like the ACoA aneurysms, from the side of the dominant $A_{1}$ in case of unequal $A_{1}$ s, and from the medial part of ACoA with equal A1s [442]. Importantly, the perforators usually arise from the superior or posterior surface of the ACoA, rarely from its anterior or inferior surface [295]. Perforators may arise from multiple, fenestrated, or even hypoplastic and anomalous branches of the ACoAs [12,232,362,423,442]. The ACoA perforators supply the infundibulum of the pituitary, optic chiasm and superior part of the optic nerve, anterior hypothalamus and lamina terminalis, anterior perforating substance, rostrum and genu of the corpus callosum, anterior commissure, anterior cingulate gyrus, parolfactory gyrus, para- terminal gyrus, septum pellucidum, and some parts of the limbic system including the column of fornix $[11,12,67,232,295,324,362,415,423$, 442]. Anastomoses between some of these perforators, in particular the hypothalamic branches, are frequent [232,362].

As aneurysms of the very proximal A2 trunk may involve the perforating branches of the ACoA, they may be endangered during dissection, coagulation, application of standard or tunnel clips, or trapping procedures, especially when dealing with wide based $\mathrm{A}_{2} \mathrm{As}$ and associated arterial anomalies [232]. Impairment of these perforators during surgery may cause a wide range of neurological symptoms including memory deficits, changes in personality, and electrolyte imbalances $[362,442]$.

\subsubsection{A2 segment}

The $A_{2}$ segment originates at the junction of the $A 1$ and the ACoA (Fig. 1). It ascends in the lamina terminalis cistern, in front of the lamina terminalis, enters the interhemispheric fissure and the callosal cistern with a course toward the

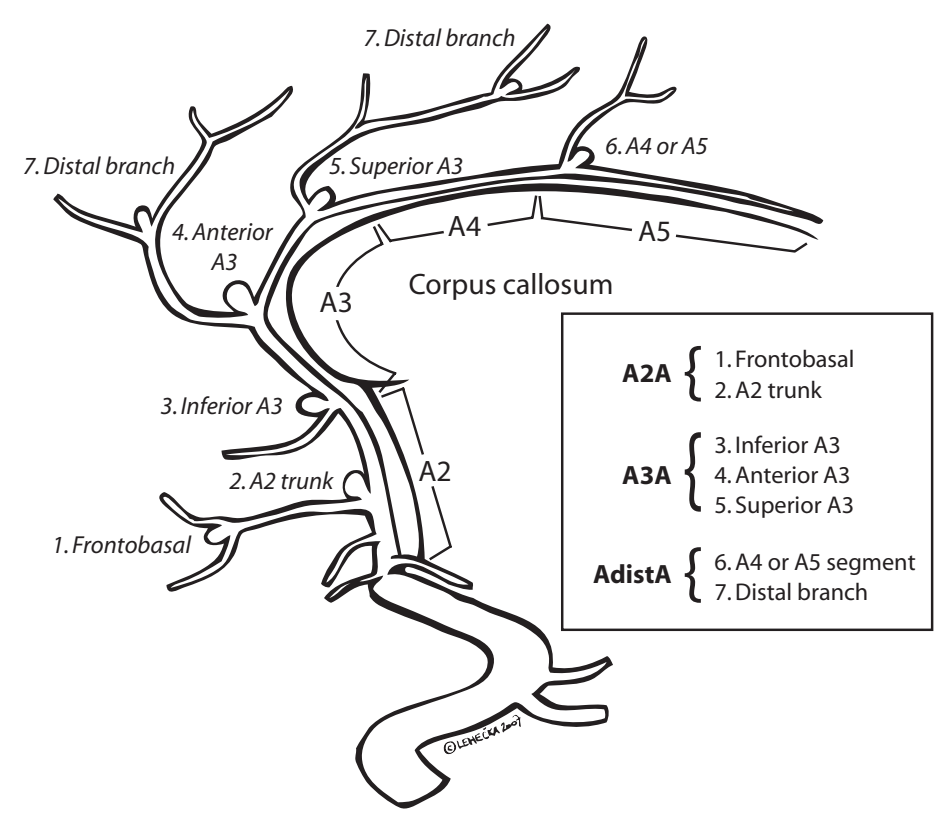

Fig. 2. Division of DACA aneurysms according to ACA segments. 
GCC (Fig. 2). The A2 terminates at the junction of the rostrum and the genu of the corpus callosum where the $A_{3}$ segment starts [294,442]. Inside the interhemispheric fissure, $A 2 s$ have been seen side by side in $18 \%$ (Fig. 3a), the left A2 anteriorly in $48 \%$, and the right $A 2$ anteriorly in $34 \%$ [295]. The free margin of the anterior falx is well above the corpus callosum. The A2 segment is entirely below the free margin which allows free shift and crossover of its branches across the midline [294]. This means that both A2 segments can be reached via a unilateral approach [122,443].

\subsubsection{Arterial branches of A2 segment}

The $A 2$ segment gives rise to three major branches: the recurrent artery of Heubner (RAH), the orbitofrontal artery (OFA), and the frontopolar artery (FPA) (Fig. 1). These arteries can be distinguished by their final destination but not by their diameters. High variation in the origin and size of these branches makes it impossible to define a standard vascular pattern for the A2s [178,294,417].

\subsubsection{Recurrent artery of Heubner (RAH)}

The origin of the RAH and its course in relation to the $A_{1}$ trunk is highly variable. In the majority of cases, it originates from the first few $\mathrm{mm}$ of the $\mathrm{A}_{2}$ segment or the $\mathrm{A}_{1}-\mathrm{A}_{2}$ junction, and in only about $10 \%$ from the distal A1 segment $[13,67,96,166,295,412,442,444]$. Rare origin from the orbitofrontal branch is also known [96]. The RAH supplies the anterior inferior striatum, anterior limb of the internal capsule, olfactory region, anterior hypothalamus (overlapping with medial lenticulostriate arteries (MLAs) from $\mathrm{A}_{1}$ ), frontobasal cortex, and subcortical white matter $[67,96,289,295,392,442]$. The RAH has been found to be missing in 3\% and duplicated in $12 \%$ in a series of 60 hemispheres [96]. The diameter varies a lot (0.2-2.9 $\mathrm{mm})$, and in rare cases the
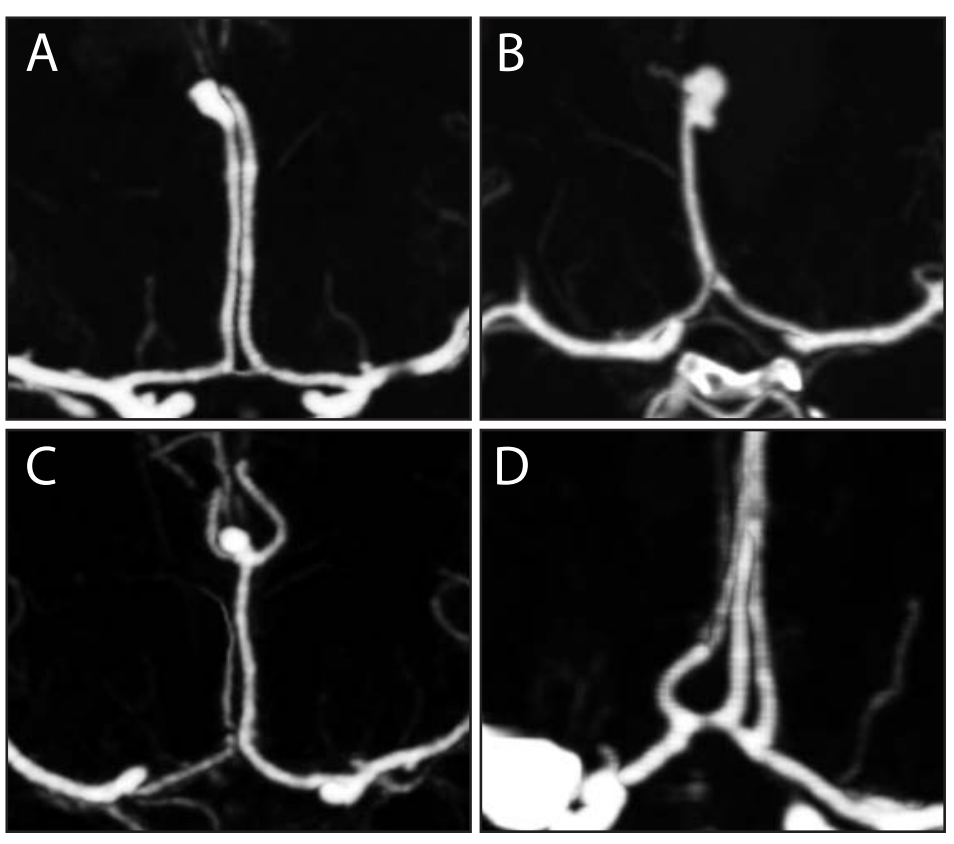

Fig. 3. Anomalies of ACA arising from the ACOA: (A) Normal configuration of $A 2 s$; (B) Azygos ACA; (C) Bihemispheric ACA; (D) Triplicated ACA.
$\mathrm{RAH}$ is as thick as the $\mathrm{A}_{1}$ [295]. Independent of its point of origin, the RAH directs immediately backward towards the $A_{1}$ segment, and then follows the superior wall, the anterior wall, or the posterior wall of the $A_{1}$ trunk. In case of the superior course (61\%), the first few mm of the RAH are often attached to the $A_{1}$ trunk by arachnoid bands, and their differentiation is of great importance before temporary clipping of $\mathrm{A}_{1}$ [96]. The distal part of the $\mathrm{RAH}$ travels freely in the subarachnoid space before penetrating into the brain at the base of the lateral and medial olfactory stria, with a highly variable branching 
pattern (1-12 branches) $[67,96,295,442]$. These perforators are often of the same caliber as the lenticulostriate arteries [11]. Consequently, it is very important to preserve the RAH to avoid ischemic injury to the anterior perforating substance.

\subsubsection{Orbitofrontal artery (OFA)}

The OFA arises more distally on the $\mathrm{A} 2$ than the RAH (Fig. 1). It generally courses downward and forward toward the floor of the anterior cranial fossa to reach the level of the planum sphenoidale. It supplies the gyrus rectus, olfactory bulb and tract, and the medial part of the orbital surface of the frontal lobe $[178,294,417]$.

\subsubsection{Frontopolar artery (FPA)}

The FPA arises after the OFA and courses anteriorly towards the medial subfrontal surface and the frontal pole (Fig. 1). It crosses the subfrontal sulcus and supplies portions of the medial and lateral surfaces of the frontal pole [324]. It is directed anteriorly rather than laterally which helps to distinguish it from the RAH. Both the OFA and the FPA can be identified in over $95 \%$ of hemispheres [417]. They do not always arise from the $A_{2}$ trunk but may also arise from the $A_{1}$ trunk or even the callosomarginal artery (CMA) [162,178,324,417].

\subsubsection{Basal perforating branches}

A2 gives also rise to small basal perforating branches which overlap the ACoA perforators. These $A 2$ perforators are usually four in number and they supply the anterior hypothalamus, septum pellucidum, medial portion of the anterior commissure, pillars of the fornix, and anteroinferior part of the striatum [294,295]. They pass posteriorly to enter the optic chiasm, lamina terminalis, and anterior forebrain below the corpus callosum [294]. The medial portions of the rostrum and the genu of the corpus cal- losum are mainly supplied by the subcallosal artery which arises from the ACoA [415].

\subsubsection{A3 segment and its branches}

The $A_{3}$ segment starts at the junction of the rostrum and the genu of the corpus callosum, curves around the genu, and terminates at the origin of the horizontal part of the ACA [79] (Fig. 2). All $A 3$ to $A 5$ segments coursed in the callosal sulcus in $60 \%$, at least one segment was found in the cingulate sulcus in 33\%, and in $7 \%$ the $A_{3}$ to $A_{5}$ segments were located in the cingulate sulcus, not involving the corpus callosum at all [415]. The $A_{3}$ segment gives origin to several cortical branches: the anterior internal frontal artery (AIFA); the middle internal frontal artery (MIFA); the posterior internal frontal artery (PIFA); and most importantly, the CMA [294]. High variation in the origin and size of these branches makes it impossible to define a standard vascular pattern for the A3s $[178,294,417]$. Some of the cortical branches may have a common trunk of origin, may arise from different segments, or may be totally absent $[178,294,417]$. The three internal frontal arteries (see above) supply the medial and lateral surfaces of the superior frontal gyrus as far posteriorly as the paracentral lobule [324]. In addition to the cortical branches, there are also thin arteries originating from the $\mathrm{A}_{3}$ to $\mathrm{A}_{5}$ segments which directly supply the superficial surface of the corpus callosum, called callosal and cingulocallosal arteries [415].

\subsubsection{Callosomarginal artery (CMA)}

The CMA is the major branch of the distal ACA with diameter of $1.8-1.9 \mathrm{~mm}$, as thick as the ACA at the same level $[294,417]$. Like other cortical branches of the ACA, the CMA cannot be defined by its branches since the usual branches of that region can arise directly from the ACA trunk as well. The CMA has been defined as the artery that courses in or near the cingulate sulcus and gives rise to two or more 
major cortical branches [257]. The CMA is totally absent in 9-18\% [162,178,294,417]. It originates most frequently (73\%) at the $\mathrm{A}_{3}$ (Fig. 1), but origins from the $A_{2}$ or the $A_{4}$ were also observed [294]. After its origin, the CMA courses in or near the cingulate sulcus and often gives rise to several cortical branches. According to the course, three types of hemispheres have been described: 1) no CMA; 2) atypical CMA, lacking the long course in the cingulate sulcus and oriented directly toward the cortex (Fig. 4a); and 3) typical CMA, running parallel to the pericallosal artery in the cingulate sulcus for a relatively long distance (Fig. 4b) [417]. The origin of the CMA is the most frequent site for the DACA aneurysms [52,122,279,312,383].

\subsubsection{A4 and $A 5$ segments and their cortical branches}

The A4 segment begins at the horizontal portion of the ACA and continues backward along the superior surface of the corpus callosum towards the splenium (Fig. 1). The virtual plane of division between the $\mathrm{A}_{4}$ and the $\mathrm{A}_{5}$ segments is the coronary suture. The most important branches originating from the $\mathrm{A}_{4}$ and A5 segments are: the posterior internal frontal artery (PIFA), the paracentral artery, the superior parietal artery, and the inferior parietal artery [294,324]. The PIFA originates as often from the $A_{3}$ segment as the $A_{4}$ segment. High variation in the origin and diameter makes it impossible to consistently classify the cortical branches of the $A_{3}$ to $A_{5}$ segments of the ACA $[178,294,417]$. The cortical branches of the $A 4$ and the A5 usually supply the posterior third of the superior frontal gyrus, part of the cingulate gyrus, a portion of the premotor, motor, and somatic sensory areas, the precuneus, and adjacent portions of the cuneus [324].

\subsubsection{Anatomic anomalies of $A C A$}

The important anomalies involving the ACA are: azygos ACA; bihemispheric ACA; trip-
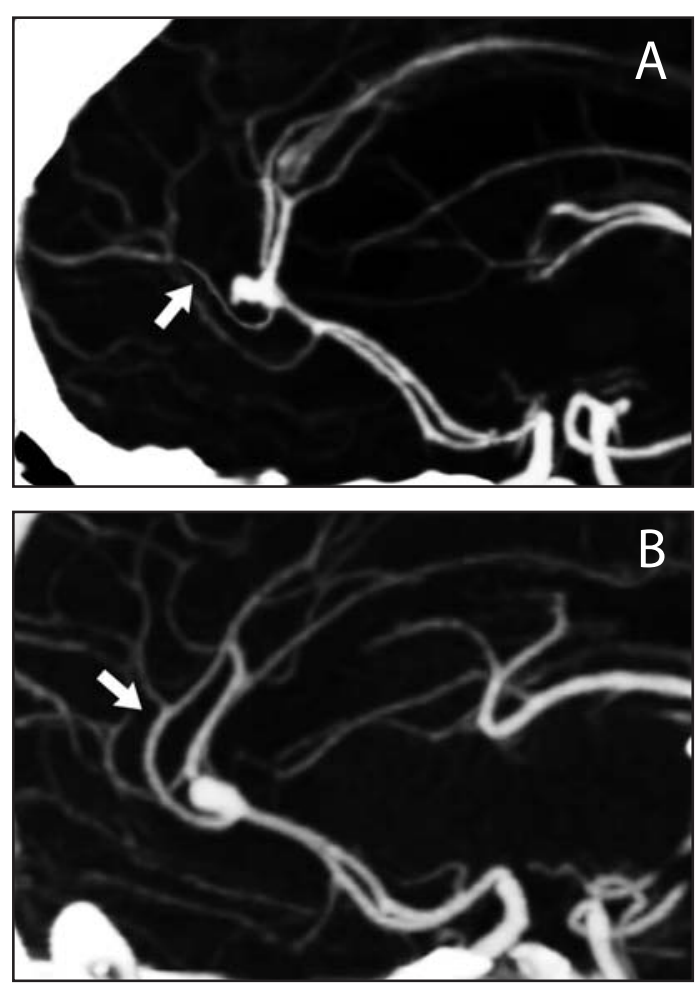

Fig. 4. (A) Atypical course of the callosomarginal artery (CMA) directly towards the cortex. (B) Typical course of the CMA in the cingulate sulcus.

lication of $A C A$; and crossover branches of the ACA $[14,97,198,216,294,442]$. The azygos ACA is a single trunk distal to the $A_{1}$ segments, so it supplies both hemispheres (Fig. $3 \mathrm{~b}$ ). In the bihemispheric ACA, one $A_{2}$ is hypoplastic and the larger $A_{2}$ gives origin to most of the cortical branches (Fig. $3 \mathrm{C}$ ). In case of ACA triplication (Fig. $3 d$ ), the middle $A 2$, also called the hemispheric type of the medial callosal artery [415], is prominent and supplies the corpus callosum. Crossover branches, found in $26-64 \%$ of patients, originate from the distal ACA and continue to the contralateral hemisphere where they supply a small medial area [294,380]. Severing of the crossover branches may cause injury to the contralateral side of the approach. In anatomic studies, the azygos ACA was seen in $0.2-4 \%$, the bihemispheric ACA in $0.2-12 \%$, 
and the triplication of ACA in 3-13\% of patients $[14,97,178,216,294,380,416,417,442]$.

\subsubsection{Interhemispheric fissure}

DACA aneurysms are located in the midline, inside the interhemispheric fissure, where the cerebral hemispheres are partially separated by the falx. The depth of the falx varies, but its free margin is well above the corpus callosum. Importantly, the $A_{2}, A_{3}$ and $A_{4}$ segments are usually below the free margin which allows free shift and crossover of their branches across the midline [294]. On the other hand, only the most anterior portions of the $A_{5}$ segment and the CMA are bellow the free margin. The cingulate gyri may be so adherent to each other that during the surgical exposure they may be mistaken for the corpus callosum [443]. The interhemispheric fissure is narrow and only a limited amount of cerebrospinal fluid (CSF) can be removed from there.

\subsubsection{Venous structures}

The frontal superficial veins are divided into three groups: lateral, medial, and inferior. In addition, each group comprises a group of ascending and a group of descending veins [280]. The veins draining the lateral convexity of the frontal lobe are either ascending to the superior sagittal sinus or descending to the superior sylvian vein. The ascending veins are frontopolar, anterior frontal, middle frontal, posterior frontal, precentral, and central veins $[280,323]$. The ascending veins of the medial frontal surface join the ascending convexity veins along the superior rim of the frontal lobe to form subdural bridging veins that empty into the superior sagittal sinus $[178,280,293,323,367]$. The de- scending veins of the medial surface drain the genu and the rostrum of the corpus callosum, and an adjacent part of the cingulate gyrus. They terminate in the inferior sagittal sinus or into the anterior cerebral vein, which joins the deep sylvian vein [323].

The bridging veins, with a diameter of 1-4 $\mathrm{mm}$, have a short free course $(5-10 \mathrm{~mm})$ in the subdural space before entering the superior sagittal sinus [323]. Bridging veins may enter the sagittal sinus directly, but they may also first join a meningeal sinus within the dura, with a course of $5-30 \mathrm{~mm}$ to the lateral angle of the sagittal sinus $[8,280]$. A single meningeal sinus may drain several cortical veins. The dura may also contain enlarged venous spaces called lacunae, extending laterally up to $3 \mathrm{~cm}$ from the midline. The lacunae predominantly drain meningeal veins before entering the sagittal sinus [280]. Most of the cortical veins pass beneath the lacunae and enter directly into the superior sagittal sinus, but a few have a common access to the sinus. The lacunae are largest in the posterior frontal region. Arachnoid granulations often protrude into the floor and walls making them adherent to the cortical surface (Fig. 5).

The bridging veins pose a potentially dangerous obstacle to the surgical approaches into

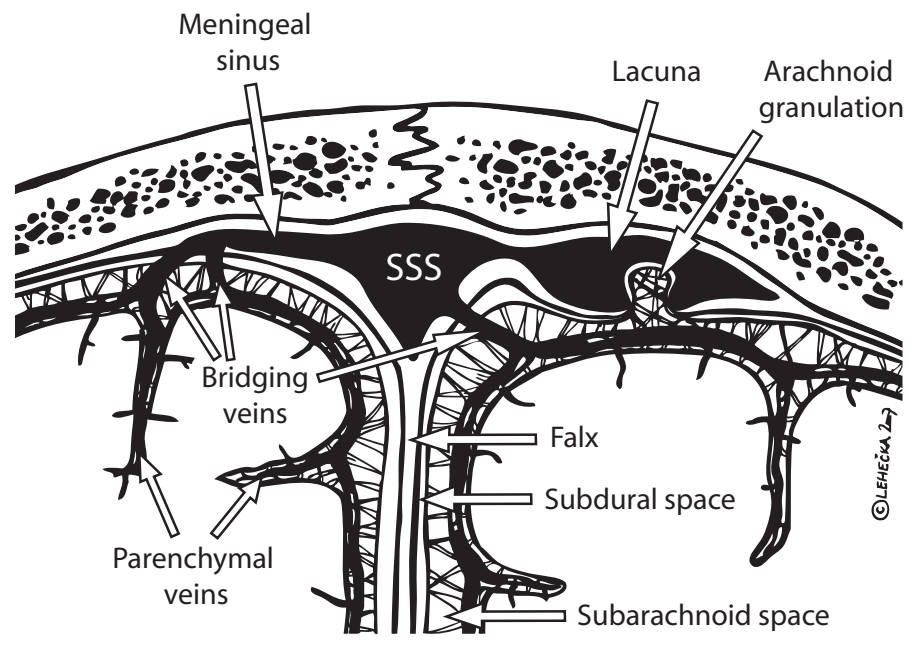

Fig. 5. The superior sagittal sinus (SSS) and its relation to venous lacunae, meningeal sinuses, and bridging veins. 
the interhemispheric space. All bridging veins should be left intact if possible, and sufficient working space should be searched in between them. The venous pattern varies highly, but often a corridor of some centimeters can be found [280]. The larger the damaged or sacrificed vein and the closer to the central sulcus it lies, the higher the risk of venous infarction with clinical symptoms. Aplastic superior sylvian vein requires special attention because of high risk of venous infarction due to reduced venous collateral flow [293].

Inside the interhemispheric fissure, one may encounter the right and left anterior pericallosal veins side by side on the corpus callosum, varying highly in size. The pericallosal veins drain the genu and rostrum of the corpus callosum and empty into the anterior end of the inferior sagittal sinus [323]. They should be left intact if possible, but there are often good venous collaterals.

\subsubsection{Corpus callosum}

The corpus callosum is the major transverse commissure connecting the cerebral hemispheres. It is divided into rostrum, genu, body, and splenium $[396,415]$. The rostrum and the genu form a connection between the anterior portion of the frontal lobes and comprise the floor and the anterior wall of the frontal horn of the lateral ventricle. The body connects the posterior portion of the frontal lobes and the parietal lobes and forms the roof of the lateral ventricles. The splenium is the most posterior part of the corpus callosum and it connects regions of the temporal and occipital lobes. The genu of the corpus callosum is semicircular or oval in the sagittal section with a mean anteroposterior diameter of $11 \mathrm{~mm}$ [178]. The anterior portion of the corpus callosum gets its blood supply from the ACA and the splenium is supplied by the posterior cerebral artery (PCA). The callosal, cingulocallosal, and long callosal arteries originating from the pericallosal artery supply directly the superficial surface of the genu
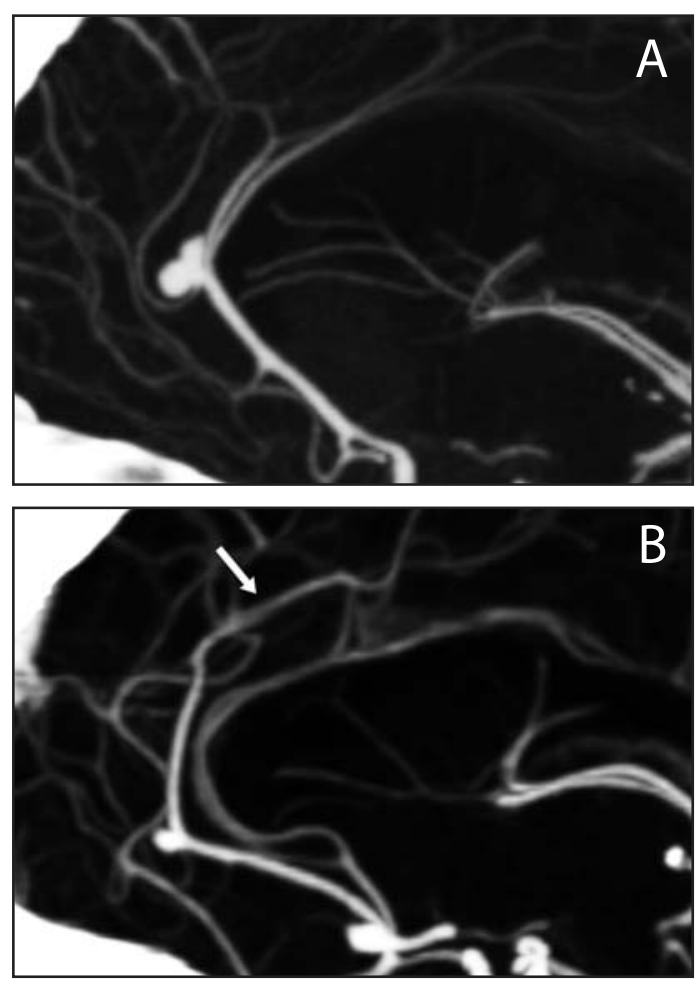

Fig. 6. The pericallosal artery coursing: (A) along the corpus callosum; (B) in the cingulate sulcus (arrow).

and the body of the corpus callosum and the cingulate gyrus [162,415].

The pericallosal artery ( $A_{2}$ to $A_{5}$ segments) travels along the corpus callosum for the majority of its course (Fig. 2). It makes a wide arch around the GCC ( $A_{3}$ segment) and then runs posteriorly along the superior aspect of the corpus callosum (Fig. 6a). Türe et al. reported an irregular course in almost $40 \%$ so that at least one of the $A_{3}$ to $A_{5}$ segments coursed in the cingulate sulcus instead of the callosal sulcus (Fig. 6b) [415]. During microsurgery the corpus callosum can be identified by its white color and parallel running transverse fibers [370]. One of the most common mistakes during interhemispheric approach is to confuse the tightly attached cingulate gyri for the corpus callosum which can lead to total loss of orientation inside the interhemipheric fissure. 


\subsection{Distal anterior cerebral artery aneurysms}

\subsubsection{Incidence and location of DACA aneu- rysms}

DACA aneurysms, also referred to as pericallosal artery aneurysms, are located distally to the $A C O A$ on the $A_{2}-A_{5}$ segments of the anterior cerebral artery (ACA) and its branches (Fig. 1)[198]. They are relatively rare, comprising about $6 \%$ (range $2-9 \%$ ) of all IAs [52,122,142, $205,248,279,312,368,383,438,445,449]$. DACA aneurysms are further divided into subgroups based on their exact locations with respect to the segments of the ACA and the corpus callosum.

\subsubsection{Aneurysms of the ACA}

Aneurysms of the ACA can be classified into five different groups: aneurysms of the $A_{1}$ segment or proximal anterior cerebral artery aneurysms ( $\left.A_{1} A s\right)$; anterior communicating artery aneurysms (ACoAAs); aneurysms of the $A_{2}$ segment and its frontobasal branches or proximal pericallosal aneurysms ( $\mathrm{A}_{2} \mathrm{As}$ ); aneurysms of the $A_{3}$ segment or classical pericallosal aneurysms ( $\left.A_{3} A s\right)$; and aneurysms of the $A_{4}$ and $A_{5}$ segments and distal cortical branches or distal pericallosal aneurysms (AdistAs) (Table 2, Fig. 2) [47]. The last three groups represent the DACA aneurysms.

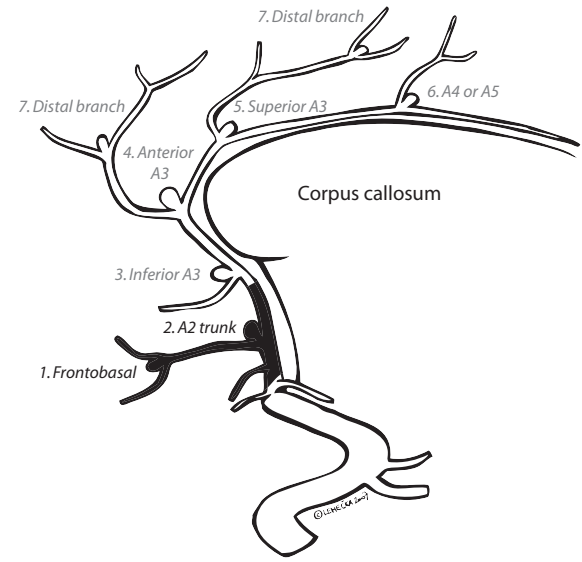

Fig. 7. Proximal DACA aneurysms (A2As).

\subsubsection{A2As (Proximal pericallosal aneurysms)}

A2As are located either directly on the pericallosal artery, between the ACoA and the genu of the corpus callosum ( $A_{2}$ segment), or on one of its frontobasal branches (Fig. 7). Proximal pericallosal artery aneurysms (A2As) are rare. The reported incidence of $A 2 A s$ has been 0.2$1 \%$ of all IAs or about $5-22 \%$ of all DACA aneurysms $[52,122,142,205,248,279,311,368,383$, $438,445,449]$. They are frequently involved with perforating arteries arising from both the ACoA and the $A 2$ segment, as well as arterial anomalies of this region $[14,294,433]$.

Table 2. The five classification categories of ACA aneurysms.

\begin{tabular}{|ll|}
\hline Category & Location \\
\hline$A_{1} A$ & A1 segment, between ICA bifurcation and ACoA \\
ACoAA & Anterior communicating artery \\
$A_{2} \mathrm{~A}$ & A2 segment and its frontobasal branches, between ACoA and genu of corpus callosum \\
A3A & A3 segment, curving around genu of corpus callosum \\
AdistA & $A_{4}$ and A5 segments or distal cortical branches such as CMA \\
\hline
\end{tabular}

CMA: Callosomarginal artery 


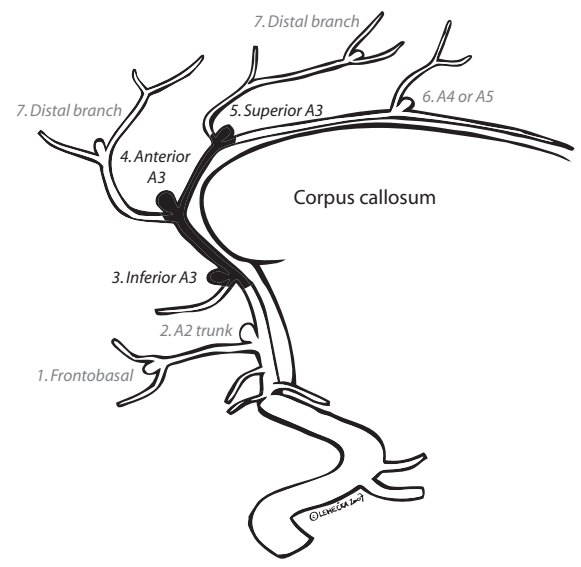

Fig. 8. Classical DACA aneurysms (A3As).

\subsubsection{A3As (Classical pericallosal aneurysms)}

$A_{3} A s$ are located at the $A_{3}$ segment of the ACA at the GCC, often at the origin of the CMA (Fig. 8). They have also been called pericallosal artery-callosomarginal artery junction aneurysms or "loco classico" pericallosal artery aneurysms. A3As are the most common of the DACA aneurysms. Their incidence is $2-7 \%$ of all IAs or $69-82 \%$ of all DACA aneurysms $[52,122,142,205,248,279,311,368,383,438,445$, 449]. A3As are difficult to reach as they lie deep in the interhemispheric fissure closely attached to the surrounding brain.

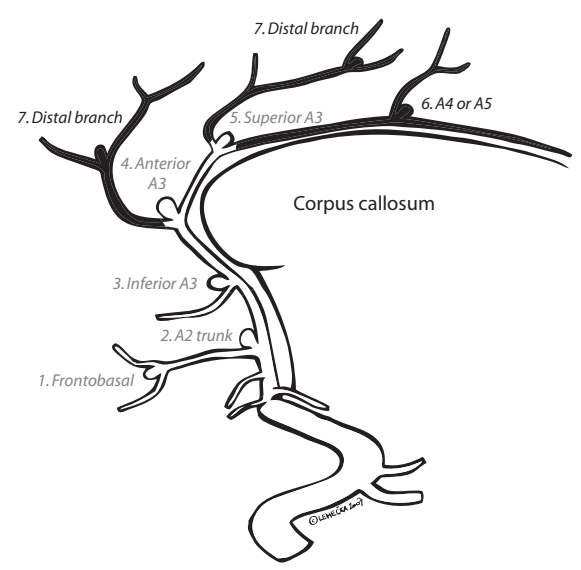

Fig. 9. Distal DACA aneurysms (AdistAs).
2.4.1.4. AdistAs (Distal pericallosal aneurysms)

AdistAs are located at the $\mathrm{A}_{4}$ and the $\mathrm{A}_{5}$ segments of the ACA, distal to the GCC, or on the cortical branches that originate from the $A_{3}$ to A5 segments, e.g., the CMA (Fig. 9). AdistAs are the least frequent of all DACA aneurysms with an incidence of only $0.3-0.6 \%$ of all IAs or $5-20 \%$ of all DACA aneurysms $[52,122,311,368$, $383,449]$.

\subsubsection{Clinical symptoms}

When DACA aneurysms rupture, they usually present with the same symptoms as seen in other ruptured aneurysms, i.e. headache, nausea, and loss of consciousness depending on the amount of bleeding [383]. Still, there are some unique neurological deficits which are more often seen in this aneurysm group, including akinetic mutism, bilateral leg weakness, behavioral changes and cognitive deficits $[33,71,87,99,382]$. These findings are thought to be related to bilateral damage to the vascular territory supplied by the distal ACA, especially the bilateral cingulate gyri and other limbic structures, and the supplementary motor area (SMA), caused either by mass effect after ICHs, or infarction [33,343].

\subsubsection{Anatomic features}

\subsubsection{Size}

DACA aneurysms are small; even when ruptured their mean size varies from 5 to $8 \mathrm{~mm} \mathrm{[32,}$ $52,122,245,264,279,368,383]$. This is somewhat less than for aneurysms located more proximal to the circle of Willis [31]. The small size of DACA aneurysms is thought to relate to Laplace's law, which states that the wall tension required to withstand a given pressure increases with the diameter of the vessel [31]. In addition, DACA aneurysms have often a broad base with possible neck calcifications and originating branches 
nearby $[52,122,205,248,279,312,368$, $383,438,443,449]$.

\subsubsection{Multiple aneurysms}

DACA aneurysms are often associated with other aneurysms $[52,122,279$, $312,443,449]$. Associated aneurysms have been reported in $25 \%$ to $55 \%$ of patients with DACA aneurysms [52, $122,142,279,312,368,376,383,438$, 443], which is higher than the usual 28-35\% reported for other aneurysm locations $[49,50,70,140,331]$.

\subsubsection{ICH and IVH}

DACA aneurysms tend to bleed into the adjacent brain tissue causing $\mathrm{ICH}$ in $17-73 \%$ of the patients [122,172, $231,312,368,376,383]$. The highest density of blood in the subarachnoid space is in the distal interhemispheric fissure, pericallosal cistern, and lamina terminalis cistern [384]. The $\mathrm{ICH}$ is usually located in the frontal lobe, corpus callosum or cingulate gyrus (Fig. 10)[143]. The high incidence of $\mathrm{ICHs}$, higher than for aneurysms elsewhere, is obviously related to the narrow pericallosal cistern and the dense attachments to the adjacent brain surface $[47,312]$. IVH is seen in $25-30 \%$ of the patients (Fig. 10a), more frequently related to ruptured $\mathrm{A}_{2} \mathrm{As}_{\mathrm{s}}$ and $\mathrm{A}_{3} \mathrm{As}$ than AdistAs [47]. Frontal ICHs are likely to cause late cognitive deficits [382].

\subsubsection{Association with ACA anomalies}

DACA aneurysms are often associated with various anomalies of the ACA $[122,383,438]$, which are thought to cause greater blood flow and shear stress on the vessel wall, and thus increase the susceptibility to aneurysm formation at the bi-, tri-, and quadrifurcations of these arteries $[10,266]$. Huber et al. reported an associa- tion between anomalies of the ACA and DACA aneurysms in an angiographic series [133]. In anatomic autopsy studies of patients without aneurysms, the azygos ACA was seen in 0.2-4\%, the bihemispheric ACA in $0.2-12 \%$ and the triplication of ACA in $3-13 \%$ of patients $[14,97,178$, $216,294,380,416,417,442]$. However, in clinical series, different anomalies of the ACA have been observed in $7-35 \%$ of patients with DACA aneurysms, an incidence generally higher than in the autopsy studies [32,142,205,383,443]. Most of the clinical observations on this association between different ACA anomalies and DACA aneurysms are based on case reports, and of these most have described the azygos ACA [399]. 


\subsubsection{Association with AVMs}

In addition to ACA anomalies, DACA aneurysms are thought to be associated with cerebral arteriovenous malformations (AVMs) $[122,383,438]$. AVMs have been reported in $3-15 \%$ of DACA aneurysm patients $[52,205,383,438]$.

\subsubsection{Special subgroups of DACA aneurysms}

\subsubsection{Giant DACA aneurysms}

Giant DACA aneurysms (diameter $\geq 25 \mathrm{~mm}$ ) are extremely rare. So far, there have been only 29 cases reported in the literature(Table 3).Only few of them were reported as part of a larger series on DACA aneurysms [52,56,122,248,376,383], most as case reports $[21,74,113,114,164,197$, $226,247,269,285,299,308,310,365,372,399,414$, 440]. Since giant DACA aneurysms may mimic the symptoms of frontal tumors, MRI and DSA are essential for reaching a correct diagnosis [215,378]. CT and CTA are helpful in identifying calcifications at the aneurysm base.

\subsubsection{Aneurysms associated with azygos ACA}

As mentioned earlier (see 2.4.3.4.) DACA aneurysms are frequently associated with anomalies of the ACA. Of such anomalies, the azygos ACA (Fig. 3b) has received the most attention. In larger series on DACA aneurysms, an azygos ACA has been observed in $3-22 \%$ of patients $[16,32,52,142,177,188,202,205,248,264,273$, $276,279,376,383]$. In addition, there exists a large number of case reports on the subject (Table 4). It seems that the incidence of azygos ACAs compared to bihemispheric ACAs in connection with DACA aneurysms has been overestimated. It is actually very difficult to distinguish between these two anomalies in the normal DSA, even with compression of the contralateral carotid artery $[14,176]$. CTA or rotational DSA
Table 3. Published cases of giant DACA aneurysms.

\begin{tabular}{|lcc|}
\hline Authors & Year & $\begin{array}{c}\text { No. of giant } \\
\text { DACA } \\
\text { aneurysms }\end{array}$ \\
\hline Snyckers and Drake [376] & 1973 & 1 \\
O'Neill et al. [285] & 1979 & 1 \\
Pia and Zierski [299] & 1979 & 1 \\
Pozzati et al. [308] & 1982 & 1 \\
Smith and Parent [372] & 1982 & 1 \\
Hayashi et al. [114] & 1985 & 2 \\
Yamagami et al. [440] & 1986 & 1 \\
Nitta et al. [269] & 1987 & 1 \\
Maiuri et al. [226] & 1990 & 1 \\
Mishima et al. [247] & 1990 & 1 \\
Hashizume et al. [113] & 1992 & 1 \\
Hernesniemi et al. [122] & 1992 & 1 \\
Preul et al. [310] & 1992 & 1 \\
Shiokawa et al. [365] & 1993 & 2 \\
Farias et al. [74] & 1997 & 1 \\
deSousa et al. [52] & 1999 & 1 \\
Kanemoto et al. [164] & 2000 & 1 \\
Koyama et al. [197] & 2000 & 1 \\
Miyazawa et al. [248] & 2000 & 1 \\
Türe et al. [414] & 2001 \\
Topsakal et al. [399] & 2003 & 1 \\
Biondi, et al. [21] & 2006 & 1 \\
Dinc et al. [56] & & 1 \\
Steven et al. [383] & & 1 \\
\hline Present series & & 1 \\
\hline
\end{tabular}

*One of the two cases was previously reported already in Hernesniemi et al. 1992 series.

give more accurate information for making the distinction between the two anomalies.

\subsubsection{Traumatic DACA aneurysms}

Aneurysms of traumatic origin represent less than $1 \%$ of all IAs $[19,172,261]$ and traumatic DACA aneurysms are even more rare [142]. Traumatic DACA aneurysms have been 
Table 4. Published cases of DACA aneurysms associated with azygos ACA.

\begin{tabular}{|c|c|c|c|}
\hline Author & Year & $\begin{array}{c}\text { Series/ } \\
\text { Case report }\end{array}$ & $\begin{array}{l}\text { No. } \\
\text { cases }\end{array}$ \\
\hline Laitinen and Snellman [205] & 1960 & Series & 3 \\
\hline Pool and Potts [305] & 1965 & Series & 3 \\
\hline Handa et al. [108] & 1971 & Case report & 1 \\
\hline Snyckers and Drake [376] & 1973 & Series & 1 \\
\hline Kinoshita and Matsukado [188] & 1975 & Series & 2 \\
\hline Quencer and Cox [313] & 1977 & Case report & 1 \\
\hline Fankhauser and Zander [73] & 1978 & Case report & 1 \\
\hline Katz et al. [176] & 1978 & Case report & 1 \\
\hline Nukui and Aiba [273] & 1978 & Series & 2 \\
\hline Becker and Newton [16] & 1979 & Series & 1 \\
\hline Kessler [183] & 1979 & Case report & 1 \\
\hline Kondo et al. [195] & 1979 & Case report & 1 \\
\hline Huber et al. [133] & 1980 & Case report & 7 \\
\hline Olbert et al. [282] & 1980 & Case report & 1 \\
\hline Fujimoto et al. [90] & 1981 & Case report & 1 \\
\hline Niizuma et al. [266] & 1981 & Case report & 2 \\
\hline Fukawa et al. [92] & 1982 & Case report & 1 \\
\hline Benedetti and Curri [18] & 1983 & Case report & 1 \\
\hline Kuwabara et al. [202] & 1984 & Series & 3 \\
\hline Abe et al. [1] & 1985 & Case report & 2 \\
\hline Hayashi et al. [114] & 1985 & Case report & 2 \\
\hline Kaneko et al. [163] & 1985 & Case report & 7 \\
\hline Kobayashi et al. [192] & 1986 & Case report & 1 \\
\hline Yamagami et al. [440] & 1986 & Case report & 1 \\
\hline Harada et al. [109] & 1987 & Case report & 1 \\
\hline Ogasawara et al. [276] & 1987 & Series & 4 \\
\hline Kawamura et al. [177] & 1988 & Series & 6 \\
\hline Lightfoote et al. [217] & 1989 & Case report & 1 \\
\hline Schick et al. [352] & 1989 & Case report & 1 \\
\hline Mishima et al. [247] & 1990 & Case report & 1 \\
\hline Nardi et al. [262] & 1990 & Case report & 2 \\
\hline Ohno et al. [279] & 1990 & Series & 3 \\
\hline Calzolari et al. [30] & 1991 & Case report & 1 \\
\hline Cinnamon et al. [34] & 1992 & Case report & 1 \\
\hline Hashizume et al. [113] & 1992 & Case report & 1 \\
\hline Traynelis et al. [404] & 1992 & Case report & 1 \\
\hline Zderkiewicz et al. [452] & 1992 & Case report & 1 \\
\hline Shiokawa et al. [365] & 1993 & Case report & 1 \\
\hline Baykal et al. [15] & 1996 & Case report & 2 \\
\hline Inci et al. [142] & 1998 & Series & 1 \\
\hline Ng et al. [264] & 1998 & Series & 3 \\
\hline Suzuki et al. [391] & 1998 & Case report & 1 \\
\hline deSousa et al. [52] & 1999 & Series & 2 \\
\hline Dietrich et al. [55] & 2000 & Case report & 1 \\
\hline Kanemoto et al. [164] & 2000 & Case report & 1 \\
\hline Miyazawa et al. [248] & 2000 & Series & 9 \\
\hline Topsakal et al. [399] & 2003 & Case report & 1 \\
\hline Auguste et al. [10] & 2004 & Case report & 4 \\
\hline Fujimoto et al. [91] & 2004 & Case report & 1 \\
\hline Chhabra et al. [32] & 2005 & Series & 3 \\
\hline Hussain et al. [137] & 2005 & Case report & 5 \\
\hline Huh et al. [134] & 2007 & Case report & 3 \\
\hline Jagetia et al. [146] & 2007 & Case report & 1 \\
\hline Steven et al. [383] & 2007 & Series & 6 \\
\hline Present series & & & 4 \\
\hline Total number & & & 120 \\
\hline
\end{tabular}

described in only some case reports (Table 5). They are typically found in young patients or children [209$211,359,450]$. The pericallosal artery is thought to be prone for formation of traumatic aneurysms after a head trauma, since the lower margin of the falx may directly damage the arterial wall of the pericallosal artery [450]. Traumatic DACA aneurysms are usually fusiform, with thin walls and poorly defined necks [37], which makes their treatment very challenging. Wrapping, proximal occlusion, excision, trapping, parent artery occlusion with preoperative bypass, and reconstruction can be considered for these aneurysms $[17,187,208,347]$. Sometimes even a preoperative bypass, side-to-side $\mathrm{A}_{3}-\mathrm{A}_{3}$ bypass or arterial translation (e.g. STA-ACA) is needed $[179,185,186,212,294]$.

\subsubsection{Imaging of DACA aneu- rysms}

Digital subtraction angiography (DSA) is still the valid gold standard in many centers [237]. Recently, multislice helical CT-angiography (CTA) has begun to replace DSA as a noninvasive, safe, and quick imaging method with sensitivity and specificity comparable to DSA in aneurysms larger than $2 \mathrm{~mm}[98,165,167,292,366$, $397,422,434,435]$. In addition, it allows disclosure of calcifications in the arterial walls and quick reconstruction of 3D images [174]. In giant or partially thrombosed DACA aneurysms, MRI is valuable for evaluating the presence and extension of intraluminal thrombus. Nowadays, 3T magnetic resonance angiography (MRA) shows good potential in iden- 
Table 5. Published cases of traumatic DACA aneurysms.

\begin{tabular}{|lcc|}
\hline Author & Year & $\begin{array}{c}\text { No. } \\
\text { cases }\end{array}$ \\
\hline Raimondi et al. [318] & 1968 & 1 \\
Umebayashi et al. [418] & 1970 & 1 \\
Acosta et al. [2] & 1972 & 1 \\
Ferry and Kempe [77] & 1972 & 1 \\
Benoit and Wortzman [19] & 1973 & 1 \\
Sadar et al. [346] & 1973 & 1 \\
Thompson et al. [395] & 1973 & 3 \\
Endo et al. [72] & 1974 & 1 \\
Amacher et al. [5] & 1975 & 1 \\
Fleischer et al. [83] & 1975 & 1 \\
Berger et al. [20] & 1976 & 1 \\
Sarwar et al. [349] & 1976 & 1 \\
Asari et al. [9] & 1977 & 1 \\
Laurent et al. [211] & 1981 & 1 \\
Jakobsson et al. [147] & 1984 & 2 \\
Nov and Cromwell [272] & 1984 & 2 \\
Amagasa et al. [6] & 1986 & 4 \\
Martin and Hummelgard [233] & 1986 & 1 \\
Nakstad et al. [261] & 1986 & 3 \\
Yuge et al. [450] & 1990 & 1 \\
Senegor [359] & 1991 & 1 \\
Opeskin [286] & 1995 & 1 \\
Raju et al. [319] & 2001 & 1 \\
Lath et al. [210] & 2002 & 1 \\
Cohen et al. [37] & 2005 & 2 \\
Dunn et al. [68] & 2007 & 1 \\
Steven et al. [383] & 2007 & 3 \\
Present series & & 1 \\
Total number & & 40 \\
\hline
\end{tabular}

tifying small details of the vasculature without the side effects of radiation $[4,227]$.

\subsubsection{Treatment of DACA aneurysms}

\subsubsection{History}

In 1948, Sugar and Tinsley were the first to perform intracranial surgery for a DACA aneurysm in a 19-year old girl [386]. Not being able to locate the aneurysm during the operation, they decided to occlude the parent pericallosal artery. The patient survived the procedure, but developed left-sided hemiparesis.
The first intracranial surgery for a DACA aneurysm in Finland was performed on June $26^{\text {th }}, 1953$ by Aarno Snellman on a 51-year old woman with a ruptured left-sided DACA aneurysm at the $A_{3}$ segment [205]. The aneurysm was clipped at the neck with Olivecrona's clip four months after the initial rupture. Transcript of the operation report is shown in Fig. 11. An interesting detail is the use of Olivecrona's clip on the parent artery for the purpose of temporary clipping. Postoperatively, the patient developed right-sided hemiparesis, and angiography showed occlusion of the distal part of the left pericallosal artery, which was probably accidentally left within the clip. The patient recovered from the hemiparesis and eventually was able to cope independently with her daily household chores. After her unfortunate death two years later, no autopsy was performed, but the death certificate stated intracranial hemorrhage as the cause of death.

In 1954, Wilson et al. published an autopsy series of 143 patients who died of SAH [433]. Ruptured DACA aneurysms were the cause of death in seven $(5 \%)$ of them, giving the first estimate of the incidence of DACA aneurysms. Later studies have shown this to be a fairly accurate figure $[52,122]$.

\subsubsection{Conservative treatment}

Conservative treatment of DACA aneurysms has a very poor prognosis. Already the first reports on DACA aneurysms, with small patient numbers, showed high mortality (50-100\%) in the conservatively treated group $[81,205,244]$. The largest series on conservative treatment of ruptured DACA aneurysms, the Cooperative Study of Intracranial Aneurysms in 1966, reported mortality rate as high as $75 \%$ at one year follow-up [267]. This result was far worse than for any other aneurysm location. In the same study, the mortality rate for surgically treated DACA aneurysm patients was 32\% [371], which was also relatively high compared to the mortality rates of $15-25 \%$ in the previous reports 


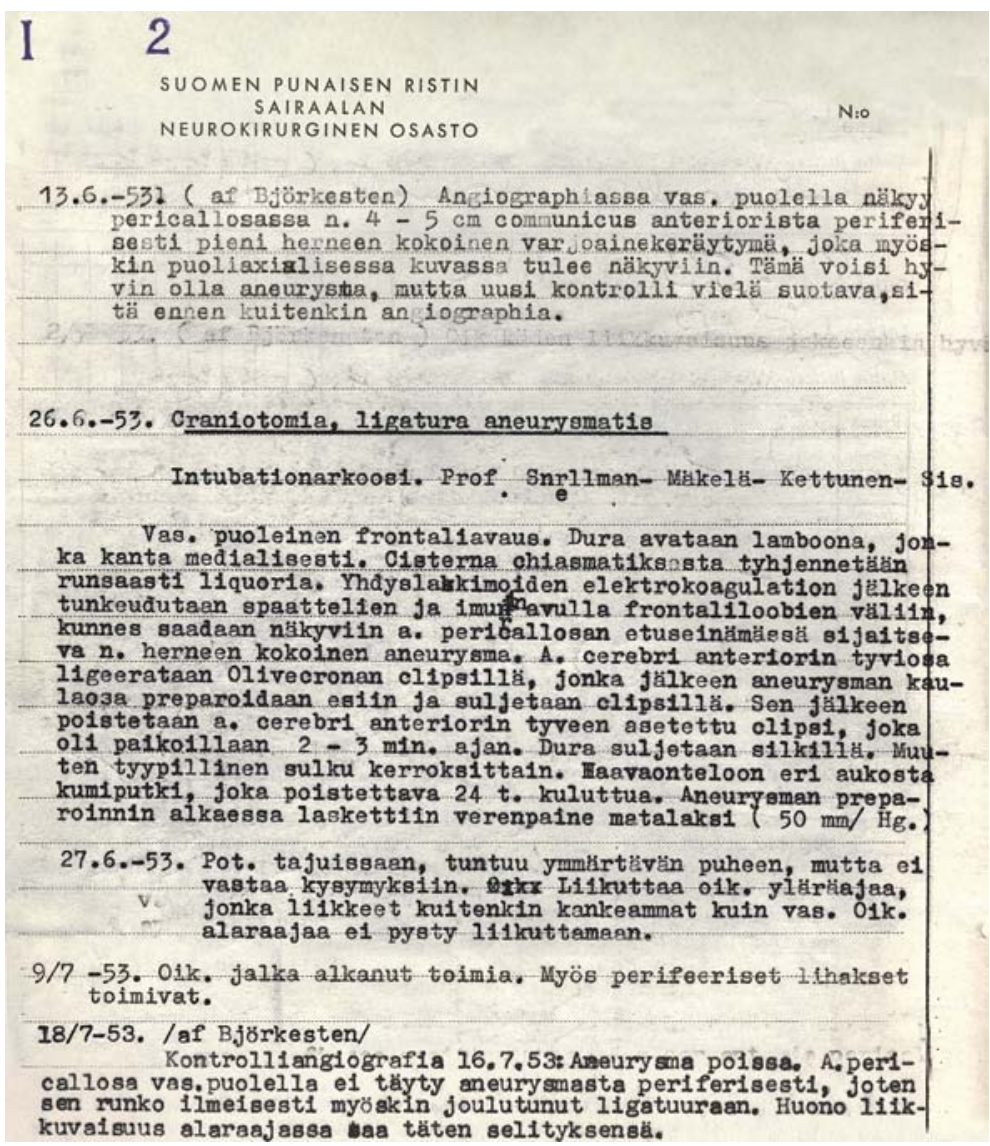

Fig. 11. Transcript of the operation report from the first DACA aneurysm surgery performed in Finland on $26^{\text {th }}$ June 1953. Translation of the report (original in Finnish):

"The Finnish Red Cross Hospital, Neurosurgical Ward

13 $3^{\text {th }}$ June 1953. (af Björkesten) On angiography, a collection of contrast medium of the size of a small pea is seen on the left pericallosal artery about 4-5 cm distally from the anterior communicating artery. This can be seen also on half-axial image. This could well be an aneurysm, but repeat control should be made. First, however, the patient should undergo angiography.

$26^{\text {th }}$ June 1953. Craniotomia, ligatura aneurysmatis Intubation anesthesia. Prof. Snellman-Mäkelä-Kettunen-Nurse

Left-sided, frontal craniotomy. Dura is opened as a single flap with the base medially. Significant amount of CSF is released from chiasmatic cistern. After electrocoagulation of the bridging veins the interhemispheric space in between the frontal lobes is entered with spatulas and suction until the aneurysm of the size of a pea is visualized on the anterior wall of the left pericallosal artery. The basal part of the anterior cerebral artery is dissected and ligated with Olivecrona's clip. The base of the aneurysm is dissected free and closed with a clip. The clip at the base on the anterior cerebral artery is then removed. It was in place for 2-3 min. Dura is closed with silk. Otherwise typical closure in layers. Rubber tube is inserted under the wound through a separate opening, it should be removed after 24 hours. At the beginning of the aneurysm dissection the blood pressure was lowered $(50 \mathrm{~mm} / \mathrm{Hg})$.

$27^{\text {th }}$ June 1953. The patient is conscious, she seems to understand what is spoken but does not answer questions. She moves her right arm, but this is clumsier than the left one. She cannot move the right leg.

$9^{\text {th }}$ July 1953. The right leg has started to function. Also the peripheral muscles function.

$18^{\text {th }}$ July 1953 (af Björkesten) Control angiography $16^{\text {th }}$ July 1953: Aneurysm is gone. The left-sided pericallosal artery does not fill distally from the aneurysm. So it seems that it is also trapped inside the clip. The poor movement of the lower limb is thus explained." 
on DACA aneurysms $[81,107,205,244,305]$. Nevertheless, after the Cooperative Study, conservative treatment has not been considered to be an option for patients with ruptured DACA aneurysms unless they are initially in a very poor clinical condition.

\subsubsection{Direct surgery before microneurosurgery}

The aneurysm series by McKissock and Walsh (1956), and Hamilton and Falconer (1959) both included DACA aneurysms as a separate group, containing seven and six patients, respectively $[107,244]$. In both of these series, DACA aneurysms were considered to be a rarity of only minor clinical importance, but at the same time they were thought to be difficult to be operated on.

The first aneurysm series dedicated to DACA aneurysms came from Laitinen and Snellman in Finland in 1960 [205]. They described their experience with 14 patients, of whom 10 were treated by direct surgery and four conservatively. Of the conservatively treated patients, two died (50\% mortality), whereas in the surgically treated group there was only one death (10\% mortality). Aneurysm occlusion was achieved either by ligating the neck with linen thread or by using silver clips. Several important features of DACA aneurysms are noted by the authors. They realized that DACA aneurysms are frequently associated with multiple aneurysms and anomalies of the ACA, and that they are firmly attached either to the falx or the cingulate gyri. In their evaluation of the surgical technique they found that the interhemispheric approach is much more suitable than the subfrontal approach for reaching the DACA aneurysms. They also noted the general difficulties in proper visualization of the aneurysm base in the deep and narrow interhemispheric fissure. In one of their patients, the aneurysm clip accidentally occluded the contralateral pericallosal artery.

Other reports on the treatment of DACA aneurysms in a small number of patients followed (Table 6) $[81,305,432]$. The Cooperative Study of Intracranial Aneurysms in 1966 had the largest number of DACA aneurysms at that time, with 26 conservatively and 39 surgically treated DACA aneurysms [371]. The mortality of 32\% in this series was rather high, but it was still slightly lower than the mortality rates for patients with aneurysms in other locations. This was the first indication that patients with ruptured DACA aneurysms may have a slightly better outcome than patients with aneurysms elsewhere.

In 1979, Yoshimoto and Suzuki presented their series of 34 DACA aneurysms operated without the operating microscope, with an exceptionally low 3\% mortality [449]. In this paper, the DACA aneurysms are divided into three groups based on the aneurysm's exact location with respect to the corpus callosum, and the authors discuss the differences in approaches to each of these locations. This paper remains the last one to report results without the use of the operating microscope.

\subsubsection{Microneurosurgery for DACA aneurysms}

Introduction of microneurosurgery was a great advance in the treatment of DACA aneurysms. Suddenly it was possible to obtain a much better visualization of both the aneurysm and the adjacent vascular structures, which meant safer and more accurate occlusion of the aneurysm base. The first report on microneurosurgical management of DACA aneurysms came from Yaşargil and Carter in 1974 [445]. The report describes 13 consecutive patients operated on by Yaşargil who started to use an operating microscope and microneurosurgical techniques in 1967. There were no deaths related to the surgery itself, but one patient died a few months later from new $\mathrm{SAH}$, this time caused by a previously unrecognized, ruptured MCA aneurysm. The general microneurosurgical technique for treatment of DACA aneurysms described in this paper is very similar to the one still in use. The major advances over time are related to better preoperative imaging, refined instruments, and certain microneurosurgical 
Table 6. Series on treatment of DACA aneurysms published before 2008.

\begin{tabular}{|c|c|c|c|c|c|c|}
\hline Author(s) & Year & $\begin{array}{l}\text { No. } \\
\text { cases }\end{array}$ & Clipped & Coiled & $\begin{array}{l}\text { Indirect } \\
\text { surgery }\end{array}$ & Conservative \\
\hline \multicolumn{7}{|l|}{ Before microneurosurgery } \\
\hline McKissock and Walsh [244] & 1956 & 7 & 4 & 0 & 1 & 2 \\
\hline Hamilton et al. [107] & 1959 & 6 & 3 & o & 3 & 0 \\
\hline Laitinen and Snellman [205] & 1960 & 14 & 9 & o & 1 & 4 \\
\hline Pool and Potts [305] & 1965 & 3 & 2 & & 1 & 0 \\
\hline Wilson et al. [432] & 1965 & 4 & 4 & o & o & 0 \\
\hline Fisher and Ciminello [81] & 1966 & 4 & 3 & 0 & o & 1 \\
\hline Skultety and Nishioka [371] & 1966 & 65 & 27 & 0 & 12 & 26 \\
\hline Dechaume et al. [53] & 1973 & 12 & 6 & 0 & 0 & 6 \\
\hline Snyckers and Drake [376] & 1973 & 24 & 15 & 0 & o & 9 \\
\hline Kinoshita and Matsukado [188] & 1975 & 10 & 8 & 0 & o & 2 \\
\hline Nukui and Aiba [273] & 1978 & 26 & 22 & 0 & o & 4 \\
\hline Becker and Newton [16] & 1979 & 12 & 9 & 0 & o & 3 \\
\hline Yoshimoto et al. [449] & 1979 & 34 & 34 & 0 & o & 0 \\
\hline \multicolumn{7}{|l|}{ Microneurosurgery } \\
\hline Yaşargil and Carter [445] & 1974 & 13 & 13 & 0 & 0 & 0 \\
\hline Kuwabara et al. [202] & 1984 & 18 & 17 & 0 & o & 1 \\
\hline Mann et al. [231] & 1984 & 11 & 11 & 0 & o & o \\
\hline Yaşargil [443] & 1984 & 23 & 23 & 0 & o & o \\
\hline Ogasawara et al. [276] & 1987 & 18 & 12 & 0 & o & 6 \\
\hline Wisoff and Flamm [438] & 1987 & 20 & 20 & 0 & o & o \\
\hline Kawamura et al. [177] & 1988 & 29 & 20 & 0 & o & 9 \\
\hline Sindou et al. [368] & 1988 & 19 & 16 & 0 & o & 3 \\
\hline Ohno et al. [279] & 1990 & 42 & 34 & 0 & o & 8 \\
\hline Fukushima et al. [93] & 1991 & 26 & 26 & 0 & o & 0 \\
\hline Hernesniemi et al. [122] & 1992 & 84 & 67 & o & o & 17 \\
\hline Martines et al. [234] & 1996 & 11 & 11 & 0 & o & 0 \\
\hline Proust et al. [312] & 1997 & 43 & 43 & 0 & 0 & 0 \\
\hline Inci, et al. [142] & 1998 & 14 & 14 & 0 & 0 & 0 \\
\hline Ng et al. [264] & 1998 & 30 & 25 & 0 & o & 5 \\
\hline deSousa, et al. [52] & 1999 & 72 & 72 & 0 & o & 0 \\
\hline Miyazawa et al. [248] & 2000 & 54 & 54 & o & o & o \\
\hline Chhabra et al. [32] & 2005 & 28 & 28 & 0 & o & o \\
\hline Dinc et al. [56] & 2006 & 26 & 24 & 1 & o & 1 \\
\hline Oshiro et al. [288] & 2007 & 20 & 20 & 0 & o & o \\
\hline Steven et al. [383] & 2007 & 59 & 58 & 1 & 0 & 0 \\
\hline \multicolumn{7}{|l|}{ Endovascular } \\
\hline Pierot et al. [300] & 1996 & 8 & 0 & 8 & o & 0 \\
\hline Menovsky et al. [245] & 2002 & 12 & 0 & 12 & o & 0 \\
\hline Keston et al. [184] & 2004 & 18 & 0 & 17 & o & 1 \\
\hline Nguyen, et al. [265] & 2007 & 26 & 0 & 25 & o & 1 \\
\hline Pandey et al. [291] & 2007 & 41 & 13 & 28 & 0 & 0 \\
\hline Present series & & 501 & 405 & 17 & 8 & 71 \\
\hline
\end{tabular}


technical nuances developed over the years by Yaşargil and others [52,122,279,443].

In the following years, a number of reports were published on microneurosurgical management of DACA aneurysms (Table 6). The fact that most of these series are small with less than 30 patients $[32,56,93,142,177,202,231,234,264$, $276,288,368,438,443$ ] shows how infrequent DACA aneurysms are and how difficult it is to obtain experience in their treatment even at one institution.

In 1990, Ohno et al. published the first of the larger DACA aneurysm series with 42 consecutive patients showing only a $2 \%$ surgical mortality [279]. Based on Yaşargil's reports [443,445], it was already evident that with microneurosurgery the mortality rates would be much lower than before, and that more focus was needed on morbidity analysis and efforts to identify patients with good or poor prognosis. Patients, divided into groups based on the Hunt and Hess classification [135], had to be evaluated separately. Ohno et al. reported good outcome in $92 \%$ of the Grade I and II patients [279]. In addition, they noted that DACA aneurysms are generally small, $94 \%$ being $\leq 10 \mathrm{~mm}$.

In 1992, Hernesniemi et al. from Kuopio Finland, published the largest series to date on DACA aneurysms [122]. They had 84 consecutive patients with 92 DACA aneurysms, of which 65 were ruptured. The main strength of this series lies in the relatively unselected patient population with only $40 \%$ of the patients initially in Grade I and II. The management mortality of $19 \%$ reflects the high number of Grade IV and V patients in the material and cannot be well compared with the highly selected previous series. On the other hand, $96 \%$ of the Grade I and II patients had favorable outcomes. These results were based on early surgery (within 72 hours) in the acute SAH phase. This paper also presents frontobasal aneurysms as a special subgroup of DACA aneurysms for the first time.

Proust et al. presented an analysis of 43 patients with DACA aneurysms, 35 of them ruptured, in 1997 [312]. They had a favorable outcome in $66 \%$ of the patients, but this was affected by the large number (26\%) of Grade IV and V patients. They concluded that the skill of the neurosurgeon is the key for successful DACA aneurysm surgery.

In 1999, deSousa et al. published a large series of 72 DACA aneurysms treated with microneurosurgery [52]. This series is highly selected, as almost all of the patients were operated on more than 10 days after SAH and there was only one Grade IV and V patient. Surgical mortality was $7 \%$ and $85 \%$ of the patients had a good outcome. Unlike in the previous series, the authors did not report the correlation between preoperative grade and outcome, but this was probably due to the patient selection and delayed surgery. However, they made an interesting observation that patients who underwent clipping of multiple aneurysms through several different approaches during the same operation had worse outcomes than those on whom only a single approach was used. This finding is in agreement with other authors with regard to the treatment of multiple aneurysms in general, possibly due to the increased risk of vasospasm [330].

Miyazawa et al. published a statistical analysis of factors affecting the outcome of DACA aneurysms in microsurgically treated patients in 2000 [248]. Their multivariate analysis showed that the preoperative grade and delayed timing of surgery were the only factors which affected the surgical outcome. This analysis did not include patients treated conservatively or any Grade $V$ patients. Furthermore, the patient population $(n=52)$ was small compared to the number of variables tested $(n=13)$ which may have affected the results.

The most recent series on microneurosurgical treatment ofDACA aneurysms was published by Steven et al. in 2007 [383]. Of the 59 patients, 36 had ruptured DACA aneurysms with a $17 \%$ management mortality and 58\% favorable outcome. Based on univariate analysis, the authors identified the clinical grade and presence or absence of ICH as the factors affecting outcome in patients with ruptured DACA aneurysms. 
2.4.6.5. Technical difficulties in DACA aneurysm surgery

Microsurgical clipping of DACA aneurysms presents certain specific difficulties when compared to other aneurysm locations as previously described by several authors [52,122,142, $231,248,264,279,312,383,438,443,445]$. Yaşargil listed these special features related to DACA aneurysms: 1) lack of working space in the interhemispheric space and pericallosal cistern; 2) dense adhesions between the cingulate gyri make separation and finding of the aneurysm difficult; 3) sclerotic wall and broad base of the aneurysm require precise positioning of the aneurysm clip; 4) origins of the branching arteries at the neck and attachment of the dome to the opposite pericallosal artery increase the risk of vascular ischemic complications; 5) difficulty in identifying lateralization of the parent artery from the preoperative images; 6 ) attachment or embedding of the dome in the pial layer of the cingulate gyrus increases the risk of tear in the aneurysm wall during dissection; and 7) with aneurysm at the bifurcation of an azygos pericallosal artery, complications may lead to vascular damage of both hemispheres [443].

Most DACA aneurysms are operated via the anterior interhemispheric route $[441,443]$. Some authors have used the bifrontal basal anterior interhemispheric approach [32,390]. Partial resection of the genu of the corpus callosum to enlarge the exposure in the infracallosal region is not advisable as it may lead to neuropsychological deficits [181]. Using the anterior interhemispheric approach, the aneurysm dome and the possible rupture site are encountered before appropriate proximal control has been achieved. This together with the dense attachments to the surrounding brain tissue are thought to result in frequent intraoperative ruptures of DACA aneurysms in $19-50 \%$ of the cases $[122,142,248,312,376$, 383].

\subsubsection{Endovascular treatment}

Clipping has been the gold standard treatment for DACA aneurysms for the past decades. However, since the first report on endovascular treatment of DACA aneurysm by Pierot et al. in 1996 [300], the number of coiled DACA aneurysms has been increasing although not to an extent comparable to other aneurysm locations. As with surgical treatment, the published series have been rather small so far (Table 6). Coiling is considered to be more demanding for DACA aneurysms than for most other aneurysms, and thereby also the complication rate has been higher $[184,265,300]$. Pierot et al. reported that only two of eight DACA aneurysms could be successfully coiled [300]. Keston et al. reported three (18\%) ruptures during coiling of 17 DACA aneurysms, and satisfactory initial packing was obtained in 14/17 (82\%) [184]. Nguyen et al. reported three (12\%) perforations in their series of 25 DACA aneurysms, one perforation causing hemorrhage resulting in transient hemiparesis, dyslexia and acalculia [265]. During the mean follow-up of 28 months, they saw aneurysm recurrence in 53\% of the patients, but there were no rebleeds. On the other hand, in all of these series, patients with SAH had a similar outcome as in most surgical series [184,245,265,291]. Pandey et al. attempted to compare the treatment results between clipping and coiling of DACA aneurysms in their series comprising 13 clipped and 28 coiled patients [291]. There was, however, a strong selection bias in the two groups which prevented any definite conclusions. Clipping and coiling were likewise compared in the International Subarachnoid Aneurysm Trial (ISAT), which included a relatively large number of DACA aneurysms (95 out of 2143 aneurysms), but due to the weakness of the subgroup post hoc analysis, no information on the possible superiority of either treatment was provided [251]. 
2.4.6.7. Technical difficulties in coiling of DACA aneurysms

It seems that, using the present technology, endovascular occlusion of DACA aneurysms is demanding because of their small size, relatively wide neck, branches originating close to the base, small caliber of the parent artery, and distal location of the aneurysm $[184,245,265,291]$. DACA aneurysms are difficult to reach via small diameter parent arteries and there is often a lack of stability and support for optimal coil deployment. Suboptimal initial coiling results easily in aneurysm recurrence [265]. As with microsurgical treatment, the result of the endovascular treatment depends very much on the experience of the surgeon, and the selection of treatment should be accordingly evaluated independently for each case. Only a long-term follow-up will indicate what is the durability of coiling in DACA aneurysms.

\subsubsection{Outcome}

\subsubsection{Short-term outcome}

Microsurgical series of patients with ruptured DACA aneurysms have reported favorable outcomes in 58-83\% and management mortality in $7-21 \%$, respectively $[52,122,231,264,279,312$, $368,383,438]$. The results vary depending on how selected the patient material is, what is the timing of the surgery, and whether the shortterm outcome is evaluated at discharge or later e.g. at one year. Some authors have suspected a less favorable prognosis for patients with DACA aneurysms compared to other aneurysm sites $[52,231]$. In other series, the outcome has been similar to that of other aneurysm locations, e.g. in the ISAT with only $11 \%$ of patients initially in Grade III to V, favorable outcome at one year was seen in $73 \%$ of the patients and mortality was $9 \%$ [250]. In general, mortality due to ruptured DACA aneurysms is unlikely to be higher than for aneurysms at other locations, but cog- nitive problems due to frontal lobe injury are more often encountered [122,382].

\subsubsection{Predictors of short-term outcome}

Only few studies have evaluated factors affecting the short-term outcome in patients with DACA aneurysms [248,291,383]. All of them have identified the initial Hunt and Hess grade as the most important factor in both univariate and multivariate analysis. A larger aneurysm size and the presence of an $\mathrm{ICH}$ are also expected to affect the outcome [248,383]. Old age, which has been shown to correlate with less favorable outcome in SAH patients in general $[207,287,329,342]$, seems to predict less favorable outcome also in patients with DACA aneurysms [291].

\subsubsection{Long-term outcome}

Relatively little is known about the longterm survival after aneurysmal SAH as only two population based studies have been published [281,337]. Major studies such as the ISAT and ISUIA reported outcomes after a mean followup of four years [251,431], other studies even earlier after the initial rupture $[105,131]$. There is no data available on the long-term outcome of patients with DACA aneurysms. One theory is that, since DACA aneurysms are located relatively distally in the cerebrovascular tree with narrow parent arteries, they might experience smaller shear stress onto the aneurysm wall making them less prone to rebleed after treatment than aneurysms at more proximal locations [427]. 


\section{Aims of the study}

I. To determine the distribution of DACA aneurysms along the different segments of distal anterior cerebral artery, and to evaluate vascular anomalies of this region.

II. To study the short-term management outcome of DACA aneurysms and to evaluate the variables influencing the outcome.

III. To assess the long-term outcome and excess-mortality in patients with ruptured DACA aneurysm compared with the normal Finnish population.

IV.-VI. To describe the present techniques in microneurosurgical management of DACA aneurysms at different locations:

IV. A2 segment of the anterior cerebral artery and the frontobasal branches (A2As)

V. A3 segment of the anterior cerebral artery ( $\left.\mathrm{A}_{3} \mathrm{As}\right)$

VI. $A_{4}$ and $A_{5}$ segments of the anterior cerebral artery and the distal cortical branches (AdistAs) 


\section{Patients, materials and methods}

This study is based on the retrospective data of 517 patients who were treated for DACA aneurysm between 1936 and 2007 at two Finnish neurosurgical units with population responsibility for the whole Southern Finland (Helsinki University Central Hospital) and Eastern Finland (Kuopio University Hospital). The combined catchment area of these two departments consists of close to 3 million people. Each part of the study ( $\mathrm{I}$ to $\mathrm{VI}$ ) included a subgroup of the whole study population (Table 7).

\subsection{Publication I: Ana- tomic features of DACA aneurysms}

\subsubsection{Patients and images}

Publication I is based on the 101 consecutive patients diagnosed with DACA aneurysm between 1998 and 2007 at the neurosurgical unit in Helsinki. The DACA aneurysms were identi-

Table 7. Basic characteristics of each subgroup of the patient population in the different parts (I to VI) of this study.

\begin{tabular}{|c|c|c|c|c|c|}
\hline Characteristic & Part I & Part Ila* & Part II $\mathbf{b}^{\dagger}$ & Part III & Part IV-VI \\
\hline Center(s) & Helsinki & Helsinki+Kuopio & Helsinki+Kuopio & Helsinki+Kuopio & Kuopio \\
\hline Time period & $1998-2007$ & 1936-1979 & $1980-2005$ & $1976-2003$ & $1977-2005$ \\
\hline No. patients & 101 & 74 & 427 & 280 & 215 \\
\hline \multicolumn{6}{|l|}{ Sex } \\
\hline Male (\%) & $27(27 \%)$ & $44(59 \%)$ & $170(40 \%)$ & $119(43 \%)$ & $104(48 \%)$ \\
\hline Female (\%) & $74(73 \%)$ & $30(41 \%)$ & $257(60 \%)$ & $161(57 \%) \%$ & $111(52 \%)$ \\
\hline Age [years]; mean (range) & $53(17-80)$ & $40(4-59)$ & $51(14-80)$ & $50(14-80)$ & $52(22-77)$ \\
\hline No. DACA aneurysms (\%) & 108 & 79 & 470 & 304 & 235 \\
\hline Ruptured & $67(62 \%)$ & $68(86 \%)$ & $277(59 \%)$ & $280(92 \%)$ & $128(54 \%)$ \\
\hline Unruptured & $41(28 \%)$ & $11(14 \%)$ & $193(41 \%)$ & $24(8 \%)$ & $107(46 \%)$ \\
\hline \multicolumn{6}{|l|}{ Aneurysm location (\%) } \\
\hline$A_{2} A$ & $7(6 \%)$ & $20(25 \%)$ & $59(13 \%)$ & $48(16 \%)$ & $35(15 \%)$ \\
\hline$A_{3} A$ & $90(83 \%)$ & $57(72 \%)$ & $377(80 \%)$ & $239(79 \%)$ & $174(74 \%)$ \\
\hline AdistA & $11(11 \%)$ & $2(3 \%)$ & $34(7 \%)$ & $17(5 \%)$ & $26(11 \%)$ \\
\hline $\begin{array}{l}\text { Aneurysm size[mm]; } \\
\text { median (range) }\end{array}$ & $6(1-35)$ & $7(3-50)$ & $6(1-41)$ & $6(2-41)$ & $5(1-40)$ \\
\hline \multicolumn{6}{|l|}{ Hunt\&Hess grade, no (\%) } \\
\hline$I-I I$ & 一 $^{\ddagger}$ & $43(59 \%)$ & $132(31 \%)$ & $140(50 \%)$ & $66(31 \%)$ \\
\hline III & - & $21(28 \%)$ & $88(21 \%)$ & $88(31 \%)$ & $41(19 \%)$ \\
\hline$I V-V$ & - & $4(5 \%)$ & $57(13 \%)$ & $52(19 \%)$ & $21(10 \%)$ \\
\hline DACA Unruptured & 一 & $6(8 \%)$ & $150(35 \%)$ & $\mathrm{o}(\mathrm{0} \%)$ & $87(40 \%)$ \\
\hline
\end{tabular}

* The historical series from 1936 to 1979 before CT and routine microneurosurgery

† From 1980 to 2005 , microneurosurgery and CT routinely available

‡ Not evaluated 
fied either by conventional DSA (Integris V3000 $1024 \times 1024$ matrix; Philips Medical Systems, Best, The Netherlands) in 39 (39\%) patients, or more recently by CTA (GE Lightspeed QX/i; GE Medical Systems, Milwaukee, WI, USA) in 62 (61\%) patients. The use of routine CTA started in year 2000 and has since become the primary imaging modality for cerebral aneurysms at this institution $[165,191]$. Four-vessel angiography was performed in 93 (92\%) patients, eight (8\%) patients had only carotid angiography.

\subsubsection{Image analysis}

All radiological data were analyzed in digital form by an experienced neurovascular radiologist (Matti Porras). All aneurysms and associated AVMs were identified in each patient. The ruptured and unruptured DACA aneurysms were divided according to their location. For each DACA aneurysm we measured the maximum dome length, maximum dome width, neck width, diameter of the parent artery, and the distance from the ACOA complex along the pericallosal artery (Fig. 12). The aneurysm dome and base with all the originating branches were evaluated. We identified anomalies of the ACA (azygos ACA, bihemispheric ACA, and triplicated $A C A$ ) in each patient. The course, dominancy,

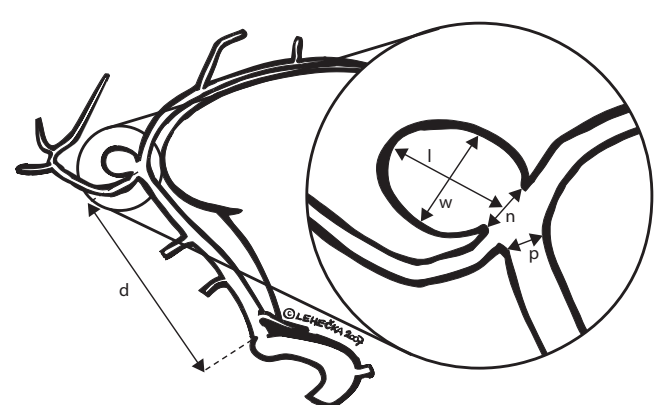

Fig. 12. Measurements from angiograms: I=dome length, $w=$ dome width, $n=$ neck width, $p=$ parent ar-tery diameter, $d=$ distance from ACoA along the pericallosal artery (in $\mathrm{mm}$ ). and origin of the branches from the pericallosal artery harboring the DACA aneurysm were evaluated.

\subsubsection{Statistical analysis}

The data were analyzed using SPSS for Windows, version 13.0.1 2004 (SPSS, Inc., Chicago, IL, USA). Categorical variables were compared using the Fisher exact two-tailed test, Pearson chi-square test, or the test for linear trend. Continuous variables were compared between groups with the Mann-Whitney U-test or the t-test.

\subsection{Publication II: Treat- ment and outcome of DACA aneurysms}

\subsubsection{Patients}

Publication II presents the combined experience of Helsinki and Kuopio neurosurgical units in the treatment of DACA aneurysms in a consecutive, retrospective series of 427 patients with 470 DACA aneurysms treated during the years 1980 to 2005 , the era of routine CT imaging and microsurgery (Part $b$ ). In addition, a historical data of 74 patients treated before year 1980 is evaluated separately for the results of premicroneurosurgical era (Part $a$ ).

The 427 patients from years 1980 to 2005 were divided into three groups: (1) 277 patients with primary SAH from a ruptured DACA aneurysm; (2) 94 patients with unruptured DACA aneurysm(s) and without acute SAH or SAH sufficiently long time ago (median 4 months) from another aneurysm; and (3) 56 patients with unruptured DACA aneurysm(s) with acute $\mathrm{SAH}$ from another aneurysm. We focused on the DACA aneurysm patients treated with microsurgery $(n=362)$ but those few treated 
with endovascular occlusion $(n=17)$ were analyzed as well. Patients described previously by Hernesniemi et al. in 1992 were reanalyzed in the present series [122].

The 277 patients with a primary rupture from a DACA aneurysm (Group 1) were compared to the 2243 consecutive patients from 1980 to 2005 with primary SAH from any ruptured aneurysm in the Kuopio Cerebral Aneurysm Database. The Kuopio Cerebral Aneurysm Database contains information about all the aneurysm patients treated from year 1977 onward at the Department of Neurosurgery, Kuopio University Hospital [47-50,119].

\subsubsection{Data collection}

Patients' clinical charts and radiological data were reviewed for relevant information. DACA aneurysms were identified by conventional DSA or recently by CTA [165]. SAH was diagnosed with CT $(n=261)$ or by lumbar puncture $(n=16)$. The initial clinical condition was graded with the Hunt and Hess $(\mathrm{H} \& \mathrm{H})$ grading system [135].

Follow-up data were collected from the day of diagnosis of a DACA aneurysm until death or December $31^{\text {st }}, 2006$. Vital status at the end of the year 2006 was ascertained from the Population Register Centre which contains information on all individuals residing in Finland. Dates and causes of death were obtained from the Statistics Finland. Outcome at one year was assessed by the Glasgow Outcome Score (GOS) [152], and further classified as favorable (GOS $\geq 4$ ) or unfavorable $(G O S<4)$. The 427 patients had a total follow-up time of 3398 patient years, with a median of 7 years (range o-24 years). No patients were lost to follow-up.

\subsubsection{Statistical analysis}

The data were analyzed using SPSS for Windows, version 13.0.1 2004 (SPSS, Inc., Chicago, IL, USA). Categorical variables were compared using the Fisher exact two-tailed test, Pearson chi-square test, or the test for linear trend. Continuous variables were compared between groups with the Mann-Whitney U-test or the t-test. Univariate association of continuous variables was tested using Spearman rank correlation coefficients.

For the 277 patients with primary SAH from a ruptured DACA aneurysm, risk factors for unfavorable outcome $(\mathrm{GOS}<4)$ at one year were analyzed using unconditional logistic regression by estimating the univariate and multivariate odds ratios (OR) with $95 \%$ confidence intervals $(\mathrm{Cl})$. The tested variables and their distribution are shown in Table 8. The maximum-likelihood stepwise forward and backward elimination procedures were used, with

Table 8. Test variables used for logistic regression analysis of unfavorable outcome after SAH from ruptured DACA aneurysm $(n=277)$ and their distribution.

\begin{tabular}{|lc|}
\hline Tested variable & Distribution \\
\hline Age [years]* & $51(14-80)$ \\
Sex; male & $110 / 277$ \\
Treatment time period [year] $^{*}$ & $1994(1980-2005)$ \\
Hypertension $^{*}$ & $88 / 274$ \\
Cardiac disease & $33 / 271$ \\
Hunt\&Hess grade $\geq 3$ & $145 / 277$ \\
Aneurysm size [mm]* & $6(2-41)$ \\
Aneurysm location at A3 segment & $224 / 277$ \\
Associated aneurysms & $96 / 277$ \\
Fisher grade $\geq 3$ & $214 / 261$ \\
ICH & $137 / 261$ \\
IVH & $90 / 261$ \\
Severe pre-op hydrocephalus & $23 / 261$ \\
Re-bleeding before treatment & $53 / 274$ \\
Delay in treatment [days]* & $2(0-105)$ \\
Treatment of multiple & $19 / 277$ \\
aneurysms in same session & $44 / 161$ \\
Use of temporary occlusion & $57 / 268$ \\
Intraoperative aneurysm rupture & $37 / 277$ \\
Ventricular drainage & $28 / 277$ \\
Permanent shunt & \\
* For continuos variable given as mean (range) \\
\end{tabular}


selection of variables based on the magnitude of their probability values $(<0.1)$. A two-tailed probability value less than 0.05 was considered significant.

\subsection{Publication III: Long- term outcome of ruptured DACA aneurysms}

\subsubsection{Patients}

In publication III we followed retrospectively the 280 consecutive patients who were treated in Helsinki and Kuopio neurosurgical units for ruptured DACA aneurysms in 19762003. Diagnosis of SAH was based on lumbar puncture before 1980 and on CT thereafter. Ruptured DACA aneurysms were identified either by DSA or recently by CTA. Clinical status on admission was expressed by the Hunt and Hess scale [135].

\subsubsection{Follow-up}

The follow-up data were collected starting from the day of diagnosis of SAH until death or December $31^{\text {st }} 2004$. Vital status at the end of year 2004 was ascertained from the Population Register Centre which contains information on all people residing in Finland. The dates and causes of death were obtained from Statistics Finland. From the same register we also obtained causes of death for the whole population of Finland during the study period. The mean follow-up time was 9.6 years (range $0.1-$ 29 yrs).

\subsubsection{Statistical analysis}

Relative survival ratio (RSR) provided a measure of the excess mortality for patients diagnosed with ruptured DACA aneurysms ir- respective of whether the excess mortality was directly or indirectly attributable to the illness [69]. The RSR was calculated by dividing the observed survival rates by the expected ones. Using the Ederer II method, the expected survival was derived from that of the comparable general population of Finland matched with respect to age, sex, and calendar time based on the data from Statistics Finland [69]. The 95\% confidence intervals $(95 \% \mathrm{Cl})$ for annual relative survival and cumulative relative survival estimates were calculated by assuming normal distribution. Statistical analysis was carried out using SAS software version 8.02 (SAS Institute Inc., Cary, NC, USA).

\subsection{Publications IV-VI: Microneurosurgical man- agement of DACA aneu- rysms}

\subsubsection{Patients (Kuopio Cerebral Aneurysm Database)}

The data presented in publications IV to VI of this study represents 3005 consecutive patients harboring 4253 IAs from the Kuopio Cerebral Aneurysm Database (1977-2005) [47-50,119]. From this database we identified 35 patients with A2As (publication IV), 163 patients with A3As (publication V), and 26 patients with AdistAs (publication VI). Prevalence of each location was calculated and other important features related to microneurosurgical treatment of these aneurysms were identified.

\subsubsection{Analysis of microneurosurgical tech- nique}

Microneurosurgical strategies for clipping of the DACA aneurysms were evaluated based 
on information obtained from the previous parts of this study (publications I to III), and on careful analysis of the microneurosurgical videos made available for this study by Professor Juha Hernesniemi, Helsinki University Central Hospital. Considerations on approaches and microsurgical techniques for clipping DACA aneurysms are discussed for each location group.

\subsubsection{Microneurosurgical videos}

We selected and edited demonstrative videos illustrating the microneurosurgical treatment of DACA aneurysms at different locations (see section List of 12 supplementary videos on microneurosurgery of DACA aneurysms). 


\section{Results}

\subsection{Incidence of DACA aneurysms (publications IV-VI)}

Of the 3005 patients in the Kuopio Cerebral Aneurysm Database, 1145 (38\%) had 1179 ACA aneurysms and 215 (7\%) patients had 235 DACA aneurysms. The 235 DACA aneurysms represented $6 \%$ of all the 4253 intracranial aneurysms. There were 35 patients with $35 \mathrm{~A} 2 \mathrm{As}$, $0.8 \%$ of all the 4253 IAs; 163 patients with 174 A3As, 4\% of all the 4253 IAs; and 26 patients with 26 AdistAs, $0.6 \%$ of all the 4253 IAs. In our series, 2365 patients presented with primary $\mathrm{SAH}$, in 855 (36\%) of them the cause was a ruptured ACA aneurysm. Of our 35 A2As, 21 (60\%) were ruptured, $0.9 \%$ of all ruptured IAs; of the $174 \mathrm{~A} 3 \mathrm{As}, 97$ (56\%) were ruptured, 4\% of all ruptured IAs; and of the 26 AdistAs, 10 (38\%) were ruptured, $0.4 \%$ of all ruptured IAs.

\subsection{Anatomy of DACA an- eurysms (publication I)}

\subsubsection{Microneurosurgical classification}

We classified the DACA aneurysms into seven groups with the genu of the corpus callosum as the anatomic landmark for the division (Fig. 2, Table 9). Aneurysms at each of these locations require modified microsurgical approaches. Table 9 and Fig. 13 present the distribution of the 108 DACA aneurysms (67 ruptured, 41 unruptured) according to their anatomic location. The $A_{3}$ segment was the most frequent with 70 $(65 \%)$ of the aneurysms anterior to the GCC, 19 (18\%) aneurysms inferior, and only one (1\%) aneurysm superior to the GCC (Fig. 14a-g). The remaining $18 \mathrm{DACA}$ aneurysms were distributed fairly evenly between the remaining locations with the frontobasal ones the least frequent

Table 9. Microneurosurgical division of DACA aneurysms and their frequencies among 108 DACA aneurysms in Helsinki from 1998 to 2007.

\begin{tabular}{|c|c|c|c|}
\hline \multirow[b]{2}{*}{ Name } & \multirow[b]{2}{*}{ Location } & \multicolumn{2}{|c|}{ Frequnecy (\%) } \\
\hline & & $\begin{array}{c}\text { Ruptured } \\
n=67\end{array}$ & $\begin{array}{l}\text { Unruptured } \\
\qquad n=41\end{array}$ \\
\hline Frontobasal aneurysms & $\begin{array}{l}\text { On the frontobasal branches } \\
\text { originating form the } A 2 \text { segment }\end{array}$ & $2 \%$ & $2 \%$ \\
\hline A2 trunk aneurysms & $\begin{array}{l}\text { Directly on the A2 segment, most often at } \\
\text { the origin of the frontopolar artery (FPA) }\end{array}$ & $4 \%$ & $5 \%$ \\
\hline Inferior $\mathrm{A}_{3}$ aneurysms & $\begin{array}{l}\text { On the proximal part of the } A 3 \text { segment } \\
\text { inferior to the genu of corpus callosum }\end{array}$ & $15 \%$ & $22 \%$ \\
\hline Anterior $\mathrm{A}_{3}$ aneurysms & $\begin{array}{l}\text { On the central part of the } A_{3} \text { segment } \\
\text { anterior to the genu of corpus callosum. }\end{array}$ & $69 \%$ & $59 \%$ \\
\hline Superior $\mathrm{A}_{3}$ aneurysms & $\begin{array}{l}\text { On the distal part of the } \mathrm{A}_{3} \text { segment } \\
\text { superior to the genu of corpus callosum }\end{array}$ & $2 \%$ & ०\% \\
\hline $\mathrm{A}_{4}$ or $\mathrm{A}_{5}$ aneurysms & On either the $A_{4}$ or $A_{5}$ segment & $4 \%$ & $10 \%$ \\
\hline Distal branch aneurysms & $\begin{array}{l}\text { On the distal cortical branches originating } \\
\text { from the } A_{3} \text { to } A_{5} \text { segments such as } \\
\text { the callosomarginal artery (CMA) }\end{array}$ & $4 \%$ & $2 \%$ \\
\hline
\end{tabular}




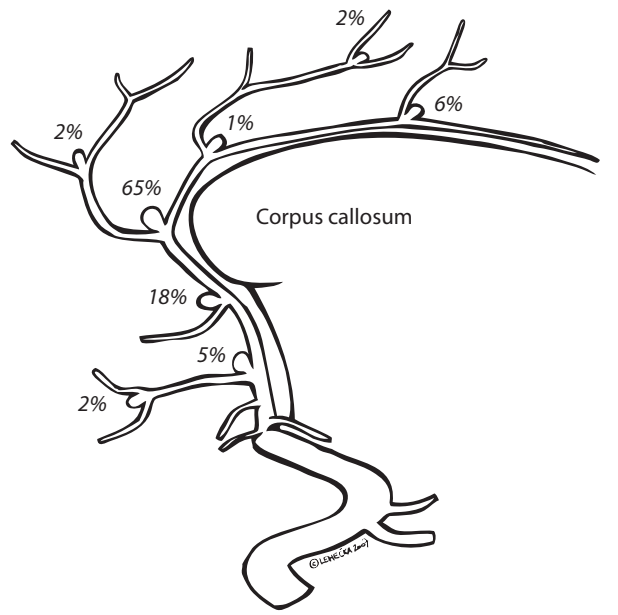

Fig. 13. Distribution of 108 DACA aneurysms based on the microneurosurgical division.

(2\%). Rupture status of the aneurysm did not affect this distribution. There were 32 ruptured and 19 unruptured aneurysms on both the left and right sides. Six (6\%) patients had either an azygos ACA or a triplicated ACA with the aneurysm in the midline. The pericallosal artery ran along the corpus callosum inside the pericallosal cistern in 97 (96\%) of the 101 patients (Fig. $6 a)$, in the remaining four (4\%) patients it was found in the cingulate sulcus for at least part of its course (Fig 6b).

\subsubsection{Aneurysm size}

The size of the DACA aneurysm was mainly dependent on its rupture status. The ruptured ones were larger than the unruptured ones with mean maximal diameters of $7.4 \mathrm{~mm}$ (range 2-35 mm) and $4.2 \mathrm{~mm}$ (range 1-9 mm), respectively, $(p<0.001)$. All size variables and measurements are presented in Table 10. Many of the aneurysms were small, 36 (54\%) of the 67 ruptured ones and as many as 34 (83\%) of the 41 unruptured ones were smaller than $7 \mathrm{~mm}$. There was no statistical significance between the size and location in either the ruptured or the unruptured aneurysms.

\subsubsection{Dome and base}

The orientation of the dome was dependent on the anatomic location of the aneurysm. In the sagittal plane, most of the frontobasal, A2 trunk and inferior $\mathrm{A}_{3}$ aneurysms had their dome directed forward. The anterior $A_{3}$ aneurysms had their dome pointing either forward or upward, and the aneurysms distal to the $\mathrm{A}_{3}$ segment had mainly backward projecting domes. On axial view, most of the aneurysms were in the midline, and only $26 \%$ were directed laterally.

The mean diameter of the DACA aneurysm neck was $2.6 \mathrm{~mm}$ (range $1-8 \mathrm{~mm}$ ), in ruptured

Table 10. Measurements of the 108 DACA aneurysms in ruptured and unruptured groups and their size distribution.

\begin{tabular}{|c|c|c|c|}
\hline & $\begin{array}{l}\text { Ruptured } \\
\qquad(n=67)\end{array}$ & $\begin{array}{l}\text { Unruptured } \\
\qquad(n=41)\end{array}$ & $\begin{array}{c}\text { Total } \\
(n=108)\end{array}$ \\
\hline Length $[\mathrm{mm}] ;$ mean $\pm \mathrm{SD}$ & $7.4 \pm 4.7$ & $4.2 \pm 2.2$ & $6.2 \pm 4.2$ \\
\hline Width $[\mathrm{mm}] ;$ mean $\pm \mathrm{SD}$ & $5.3 \pm 3.2$ & $3.2 \pm 1.6$ & $4.6 \pm 2.9$ \\
\hline Neck [mm]; mean \pm SD & $2.8 \pm 1.3$ & $2.4 \pm 0.8$ & $2.6 \pm 1.2$ \\
\hline Parent artery diameter $[\mathrm{mm}] ;$ mean $\pm S D$ & $1.8 \pm 0.4$ & $1.7 \pm 0.5$ & $1.8 \pm 0.4$ \\
\hline Neck to width ratio; mean \pm SD & $0.58 \pm 0.22$ & $0.80 \pm 0.20$ & $0.66 \pm 0.24$ \\
\hline Neck to parent artery ratio; mean \pm SD & $1.6 \pm 0.7$ & $1.5 \pm 0.7$ & $1.5 \pm 0.7$ \\
\hline \multicolumn{4}{|l|}{ Size distribution } \\
\hline Small (<7mm); n (\%) & $36(54 \%)$ & $34(83 \%)$ & $70(65 \%)$ \\
\hline Medium (7-14mm); n (\%) & $27(40 \%)$ & $7(17 \%)$ & $34(31 \%)$ \\
\hline Large (15-24mm); n (\%) & $3(4 \%)$ & 0 & $3(3 \%)$ \\
\hline Giant ( $\geq 25 \mathrm{~mm}) ; \mathrm{n}(\%)$ & $1(2 \%)$ & 0 & $1(1 \%)$ \\
\hline
\end{tabular}



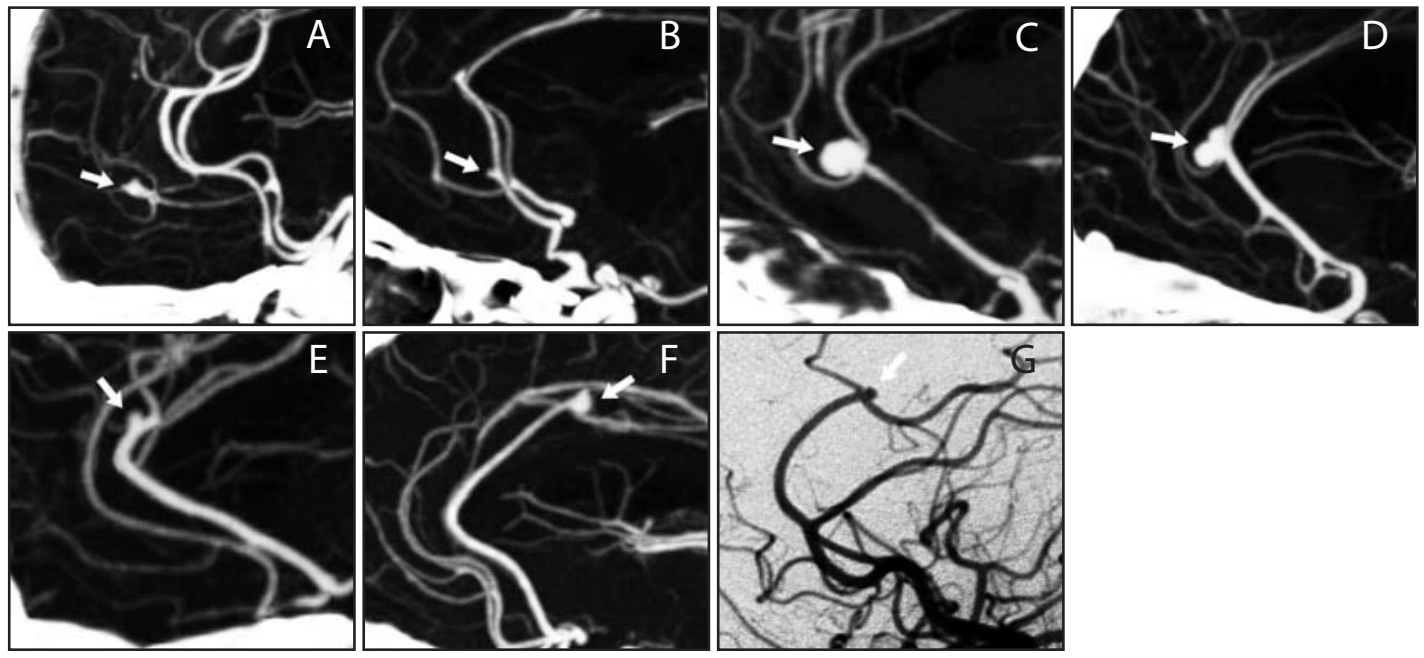

Fig. 14. CTA and DSA images of DACA aneurysms at different locations based on microneurosurgical division, arrow marks the aneurysm; (A) Frontobasal, (B) A2 trunk, (C) Inferior A3 segment, (D) Anterior $A_{3}$ segment, (E) Superior $A_{3}$ segment, (F) A4 or A5 segments, (G) Distal branches (distal callosomarginal artery).

ones slightly wider ( $0.4 \mathrm{~mm}$ ) than in the unruptured ones (Table 10). The neck-to-width ratio was larger for the unruptured aneurysms than for the ruptured aneurysms with mean values of 0.8 (1:1.3) and $0.6(1: 1.7)$, respectively. In $81 \%$ of all the aneurysms the neck-to-dome ratio was more than 1:2, with $25 \%$ of them having their neck equal to the dome width (1:1). The mean parent artery diameter was $1.8 \mathrm{~mm}$ (range 1.0-3.0 $\mathrm{mm}$ ) for both the ruptured and unruptured aneurysms. In $68 \%$, the neck was wider than the parent artery.

In only six (6\%) patients, the aneurysm base was free of originating branches, while the remaining 95 (94\%) patients had one or more branches originating from the base. $\mathrm{A}_{3} \mathrm{As}$ had most often branch origins at their base, in 99\% of cases. The CMA was the originating artery in $85 \%$, in $12 \%$ the aneurysm was at the bifurcation of an azygos ACA, bihemispheric ACA or triplicated $A C A$, and in $3 \%$ the originating branch was some small artery other than the CMA.

\subsubsection{Multiple aneurysms}

Multiple aneurysms in general were present in $50(50 \%)$ patients including seven $(7 \%)$ with multiple DACA aneurysms. The distribution of the associated aneurysms is presented in Table 11. The most frequent site for associated aneurysms was the MCA bifurcation with 42 (38\%) of the 111 associated aneurysms, a typical finding in a Finnish population with a higher prevalence of MCA aneurysms than elsewhere [49,329].

Table 11. Distribution of 111 associated aneurysms in 50 patients with multiple aneurysms.

\begin{tabular}{|cc|}
\hline Location & Number of aneurysms (\%) \\
\hline ICA & $16(14 \%)$ \\
ACoA & $11(10 \%)$ \\
MCA & $67(60 \%)$ \\
VBA & $10(9 \%)$ \\
DACA & $7(6 \%)$ \\
\hline
\end{tabular}



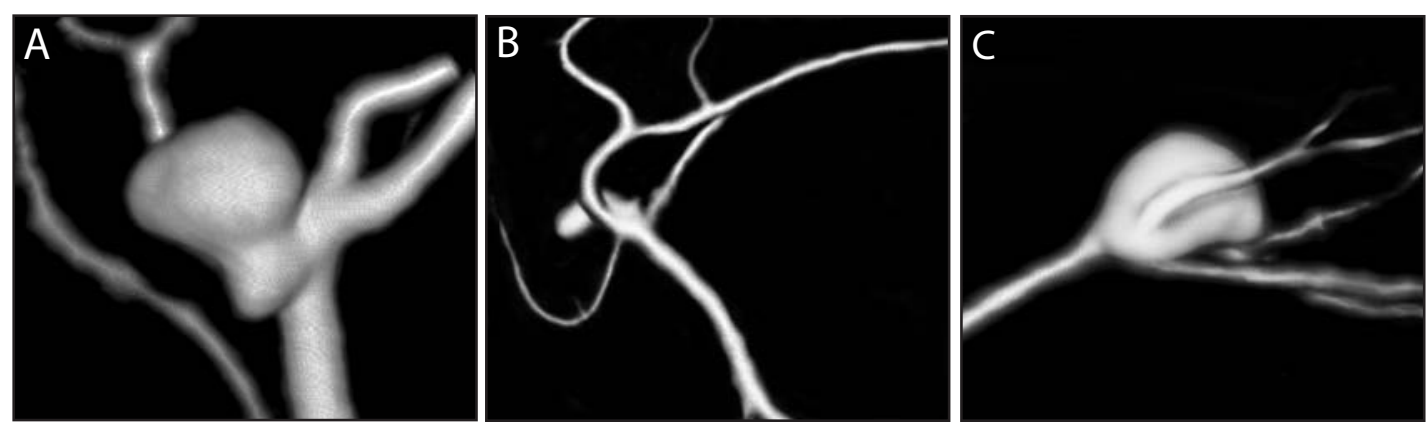

Fig. 15. (A) Anterior $A 3$ aneurysm proximal to the main bifurcation of bihemispheric $A C A ;(B)$ Anterior $A_{3}$ aneurysm at the main bifurcation of azygos $A C A$ with three branches at the base; (C) A4 aneurysm at the bifurcation of triplicated ACA with three braches originating at the base.

\subsubsection{Anomalies of ACA}

A normal course of $A 2$ segments, showing two arteries of similar size running parallel, was observed in 78 (77\%) patients (Fig. 3a). The remaining $23(23 \%)$ patients presented various anomalies of the A2 segment (Table 12).

\subsubsection{Azygos $A C A$}

A true azygos ACA (Fig. 3b) was present in four (4\%) patients. In all of these four patients the DACA aneurysm was located anterior to the

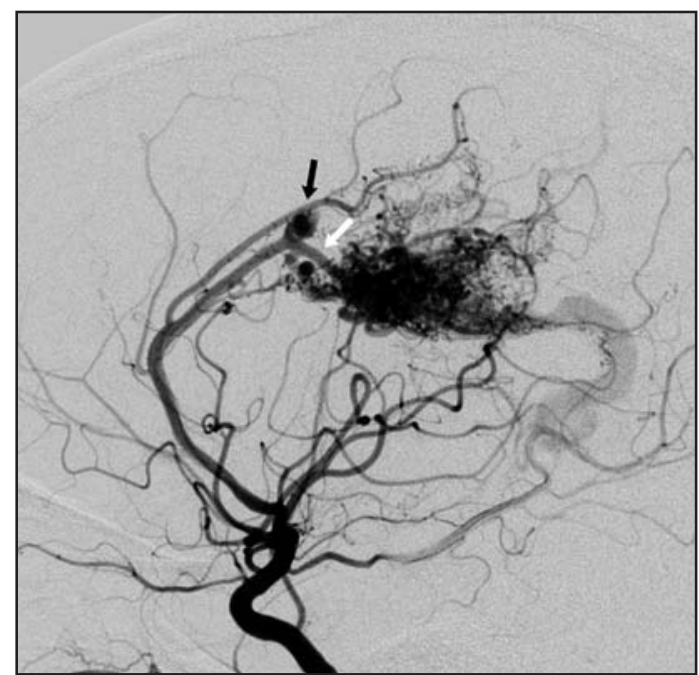

Fig. 16. AVM associated with DACA aneurysm (black arrow); the main feeder is the pericallosal artery (white arrow).
Table 12. Anomalies of the anterior cerebral artery (ACA) in the 101 patients with DACA aneurysms.

\begin{tabular}{|lc|}
\hline Type of ACA & No. (\%) \\
\hline Normal anatomy & $78(77 \%)$ \\
Anomalies & $23(23 \%)$ \\
Azygos ACA & $4(4 \%)$ \\
Bihemispheric ACA & $15(15 \%)$ \\
Triplication of ACA & $4(4 \%)$ \\
\hline
\end{tabular}

genu at the bifurcation of the azygos pericallosal artery.

\subsubsection{Bihemispheric ACA}

A bihemispheric ACA (Fig. 3C), the most frequent anomaly of the $A 2$ segment, was seen in 15 (15\%) patients. Unlike with the azygos ACA, aneurysms associated with a bihemispheric ACA were not always found at this bifurcation. Only nine $(60 \%)$ of the 15 patients had their DACA aneurysm at the main bifurcation of the bihemispheric ACA (Fig. 15b), the other six (40\%) were either proximal or distal to this point (Fig. 15a).

\subsubsection{Triplication of $A C A$}

Triplication of the ACA (Fig. 3d) was seen in four (4\%) patients. In all four patients, it was the third artery in the midline which harbored 
the DACA aneurysm. This artery ran along the corpus callosum and divided into the left and right branches at the aneurysm (Fig. 15C). Two of the aneurysms were located at the $A_{3}$ segment and two at the $A_{4}$ segment, more distally than for the azygos ACA.

\subsubsection{Associated AVMs}

We had only one (1\%) patient with DACA aneurysm and associated AVM among the 101 patients (Fig. 16).

\subsection{SAH from ruptured DACA aneurysm (publi- cation II, part b)}

\subsubsection{Age, presentation, and clinical condition}

There were 277 patients with primary SAH from a ruptured DACA aneurysm. They were compared to the 2243 consecutive patients with primary SAH from an aneurysm of any location in Table 13. In both series, women somewhat predominated and men bled somewhat earlier. There was a similar initial rebleeding rate in both genders. The more frequent ICHs from ruptured DACA aneurysms did not correlate with the Hunt and Hess grades on admission in either of the two groups (Table 13).

\subsubsection{Radiological features}

\subsubsection{CT findings}

The ruptured DACA aneurysms presented very often with ICH on the initial CT scan (Fig. 10a), in as many as $53 \%$ of them, but there were no differences in IVH or severe preoperative hydrocephalus rates when compared to ruptured aneurysms in other locations (Table 13).
Table 13. Characteristics and comparison of findings in (1) 277 ruptured DACA aneurysms and (2) all consecutive, ruptured aneurysms in the Kuopio Cerebral Aneurysm Data Base $(n=2243)$ among patients admitted between 1980-2005.

\begin{tabular}{|c|c|c|}
\hline & $\begin{array}{l}\text { DACA an- } \\
\text { eurysms }\end{array}$ & $\begin{array}{l}\text { All aneu- } \\
\text { rysms }\end{array}$ \\
\hline No. of patients & 277 & 2243 \\
\hline Single aneurysm & $181(65 \%)$ & $1840(82 \%)$ \\
\hline Multiple aneurysms & $96(35 \%)$ & $403(18 \%)$ \\
\hline \multicolumn{3}{|l|}{ Sex } \\
\hline Male & $110(40 \%)$ & $1059(47 \%)$ \\
\hline Female & $167(60 \%)$ & $1184(53 \%)$ \\
\hline Age [years]; mean (range) & $51(14-80)$ & $51(5-95)$ \\
\hline Male & $48(14-75)$ & $48(10-86)$ \\
\hline Female & $53(30-80)$ & $54(5-95)$ \\
\hline Re-bleeding before treatment & $53(18 \%)$ & $380(17 \%)$ \\
\hline $\begin{array}{l}\text { Aneurysm size }[\mathrm{mm}] \\
\text { median (range) }\end{array}$ & $6(2-41)$ & $8(1-60)$ \\
\hline Small aneurysms $(<7 m m)$ & $142(51 \%)$ & $936(42 \%)$ \\
\hline \multicolumn{3}{|l|}{ Blood on initial CT scan } \\
\hline No scan done & $16(6 \%)$ & $162(7 \%)$ \\
\hline Fisher grade 1 & $9(3 \%)$ & $71(3 \%)$ \\
\hline Fisher grade 2 & $38(14 \%)$ & $503(22 \%)$ \\
\hline Fisher grade 3 & $52(18 \%)$ & $680(30 \%)$ \\
\hline Fisher grade 4 & $162(58 \%)$ & $827(37 \%)$ \\
\hline \multicolumn{3}{|l|}{ ICHs and IVHs } \\
\hline Both ICH and IVH & $65(25 \%)$ & $242(12 \%)$ \\
\hline ICH only & $72(28 \%)$ & $296(14 \%)$ \\
\hline IVHonly & $25(10 \%)$ & $289(14 \%)$ \\
\hline Neither ICH or IVH & $109(42 \%)$ & $1254(60 \%)$ \\
\hline \multicolumn{3}{|l|}{$\mathrm{ICH}$ location } \\
\hline Frontal & $131(96 \%)$ & $212(39 \%)$ \\
\hline Parietal & $4(3 \%)$ & $4(1 \%)$ \\
\hline Temporal & $2(1 \%)$ & $322(60 \%)$ \\
\hline Subdural hematoma & $6(2 \%)$ & $21(1 \%)$ \\
\hline Preoperative hydrocephalus & $88(34 \%)$ & $913(44 \%)$ \\
\hline Severe & $23(8 \%)$ & $178(9 \%)$ \\
\hline \multicolumn{3}{|l|}{$\begin{array}{l}\text { Hunt and Hess grade } \\
\text { on admission }\end{array}$} \\
\hline Grade 1 & $41(15 \%)$ & $224(10 \%)$ \\
\hline Grade 2 & $91(33 \%)$ & $916(41 \%)$ \\
\hline Grade 3 & $88(32 \%)$ & $600(27 \%)$ \\
\hline Grade 4 & $31(11 \%)$ & $273(12 \%)$ \\
\hline Grade 5 & $26(9 \%)$ & $230(10 \%)$ \\
\hline \multicolumn{3}{|l|}{ Treatment type } \\
\hline Clipping & $254(92 \%)$ & $1664(74 \%)$ \\
\hline Coiling & $12(4 \%)$ & $275(12 \%)$ \\
\hline Conservative & $7(3 \%)$ & $235(10 \%)$ \\
\hline Other microsurgical & $4(1 \%)$ & $69(3 \%)$ \\
\hline
\end{tabular}


5.3.2.2. Angiographic findings (DSA, CTA, MRA)

The 277 ruptured DACA aneurysms occurred most frequently (81\%) on the $A_{3}$ segment of the ACA. The $A_{2} A s$ were somewhat more often ruptured than the AdistAs. Almost all (98\%) ruptured DACA aneurysms were saccular, and there were only three (1\%) fusiform aneurysms, one (0.4\%) traumatic, and two (0.7\%) mycotic ones. The ruptured DACA aneurysms were smaller than ruptured aneurysms elsewhere, with median diameters of $6 \mathrm{~mm}$ and $8 \mathrm{~mm}$, respectively (Table 13).

\subsection{Treatment and out- come of DACA aneurysms (publication II)}

\subsubsection{Ruptured DACA aneurysms 1936- 1979 (publication II, part a)}

There were 74 patients with 79 DACA aneurysms treated at our institutions in Helsinki and Kuopio before the year 1980, and 68 (92\%) of these aneurysms were ruptured (Table 14). On admission, most of the patients were relatively young (mean age 40 years) and in good clinical condition. At that time surgery was often postponed (median 19 days from the primary SAH) to allow recovery before surgery. With the surgical mortality of only $1 \%$ and the management mortality of $13 \%$, this historical series suggests that the surgery of ruptured DACA aneurysms becomes safer when postponed by weeks - although the overall benefit is reduced by early rebleeds as shown in later studies [278]. It also shows how much the results of surgical series on IAs depend on patient selection and how difficult it is to compare results between different series.

\subsubsection{Ruptured DACA aneurysms 1980- 2005 (publication II, part b)}

Of the 277 ruptured DACA aneurysms, 258 were treated with microneurosurgery, 12 with endovascular coiling, and seven conservatively due to initially poor clinical condition (Table 13).

\subsubsection{Microneurosurgical clipping}

Direct clipping was performed in 254 (98\%) patients, proximal occlusion of the parent artery in three (1\%) patients, and trapping in one (0.4\%) patient. Median time from rupture to surgery was two days (range 5 hrs-105 days). The frontal interhemispheric route was used in the majority of the clipped DACA aneurysms [122], and only very proximal A2 segment an-

Table 14. Characteristics of 68 patients with ruptured DACA aneurysms treated during 1936-1979.

\begin{tabular}{|ll|}
\hline Number of patients & 68 \\
Sex & \\
$\quad$ Male & $41(60 \%)$ \\
Female & $27(40 \%)$ \\
Age at diagnosis [years]; mean (range) & $40(4-59)$ \\
Time from rupture to treat- & $19(0-296)$ \\
ment [days]; median (range) & $7(3-50)$ \\
Aneurysm size [mm]; median (range) & \\
Clinical condition on admission & \\
$\quad$ Hunt\&Hess grade 1-2 & $44(65 \%)$ \\
Hunt\&Hess grade 3-5 & $24(35 \%)$ \\
Treatment & \\
Clipping & $52(76 \%)$ \\
Wrapping & $1(1 \%)$ \\
Proximal occlusion & $2(3 \%)$ \\
Explored only & $4(6 \%)$ \\
Conservative & $9(13 \%)$ \\
Surgical mortality & $1(1 \%)$ \\
Surgical morbidity & $8(12 \%)$ \\
Outcome at 1-year & \\
Favorable outcome (GOS $\geq 4)$ & $53(78 \%)$ \\
Dead & $9(13 \%)$ \\
\hline
\end{tabular}


eurysms were clipped using the pterional approach or the lateral supraorbital approach [120]. The average operation time from skinto-skin was two hours (range 45min-8hours). Postoperative angiography, not routine in the early series, was performed in 189 (73\%) of the 258 patients. Total occlusion was seen in 172 (91\%) patients, neck remnant in $10(5 \%)$, and partial or total aneurysm filling in seven (4\%) after the first surgery (Table 15). Morbidity due to treatment was seen in $38(15 \%)$ patients, in most cases a reversible neurological deficit occurred, such as paresis in the lower extremity. Treatment mortality was $0.4 \%$, as one patient died due to postoperative hematoma and brain infarction. The morbidity and mortality rates of microsurgical treatment were equal with the unruptured DACA aneurysms and ruptured aneurysms in general (Table 15).

\subsubsection{Coiling}

Only 12 of the ruptured DACA aneurysms were primarily coiled, with seven of them totally occluded (Table 15). Four patients underwent re-coiling and finally three of them underwent clipping. There was no treatment-related morbidity or mortality in this subgroup of 12 patients.

Table 15. Treatment details in five different patient groups treated between 1980 and 2005.

\begin{tabular}{|c|c|c|c|c|c|}
\hline & \multirow{2}{*}{\multicolumn{2}{|c|}{ Ruptured DACAAs, }} & \multicolumn{2}{|c|}{ Unruptured DACAAs, } & \multirow{3}{*}{$\begin{array}{l}\text { All ruptured } \\
\text { aneurysms, } \\
\text { microsurgery }\end{array}$} \\
\hline & & & \multirow{2}{*}{$\begin{array}{c}\text { no SAH } \\
\text { microsurgery }\end{array}$} & \multirow{2}{*}{$\begin{array}{c}\text { acute SAH } \\
\text { microsurgery }\end{array}$} & \\
\hline & microsurgery & endovascular & & & \\
\hline No. of patients & 258 & 12 & 84 & 20 & 1664 \\
\hline \multicolumn{6}{|l|}{$\begin{array}{l}\text { Occlusion grade after } \\
\text { treatment }\end{array}$} \\
\hline Total occlusion & $172(67 \%)$ & $7(58 \%)$ & $58(69 \%)$ & $12(60 \%)$ & $708(43 \%)$ \\
\hline Neck remnant & $10(4 \%)$ & $3(25 \%)$ & $2(2 \%)$ & 0 & $67(4 \%)$ \\
\hline Part of aneurysm filling & $6(2 \%)$ & o & $1(1 \%)$ & 0 & $25(2 \%)$ \\
\hline Whole aneurysm filling & $1(0.4 \%)$ & $2(17 \%)$ & $3(4 \%)$ & $1(5 \%)$ & $56(3 \%)$ \\
\hline No angiography control & $69(27 \%)$ & 0 & $20(24 \%)$ & $7(35 \%)$ & $808(49 \%)$ \\
\hline Ventricular drainage & $37(14 \%)$ & $1(8 \%)$ & $2(2 \%)$ & 0 & $290(17 \%)$ \\
\hline Permanent shunt & $28(11 \%)$ & o & $3(4 \%)$ & $1(5 \%)$ & $199(12 \%)$ \\
\hline \multicolumn{6}{|l|}{ Treatment complications } \\
\hline Intraoperative rupture & $57(22 \%)$ & 0 & $6(7 \%)$ & $7(35 \%)$ & $445(27 \%)$ \\
\hline Re-clipping or re-coiling & $5(2 \%)$ & $4(33 \%)$ & $2(2 \%)$ & $1(5 \%)$ & $42(3 \%)$ \\
\hline Clipping after failed coiling & - & $3(25 \%)$ & - & - & - \\
\hline Epidural hematoma & 0 & 0 & $1(1 \%)$ & 0 & $5(0.3 \%)$ \\
\hline Subdural hematoma & $3(1 \%)$ & o & 0 & $1(5 \%)$ & $16(1 \%)$ \\
\hline Postoperative ICH & $7(3 \%)$ & 0 & $3(4 \%)$ & $2(10 \%)$ & $27(2 \%)$ \\
\hline $\begin{array}{l}\text { Meningitis, non-bac- } \\
\text { terial included }\end{array}$ & $24(9 \%)$ & o & $1(1 \%)$ & 0 & $111(6 \%)$ \\
\hline Superficial wound infection & $13(5 \%)$ & o & $2(2 \%)$ & 0 & $35(2 \%)$ \\
\hline $\begin{array}{l}\text { Morbidity caused } \\
\text { by treatment }\end{array}$ & $38(15 \%)$ & 0 & $10(12 \%)$ & $3(15 \%)$ & $280(17 \%)$ \\
\hline Mortality caused by treatment & $1(0.4 \%)$ & 0 & $1(1 \%)$ & 0 & $8(0.5 \%)$ \\
\hline \multicolumn{6}{|l|}{ Outcome at 1-year } \\
\hline Favorable (GOS $\geq 4$ ) & $193(75 \%)$ & $11(92 \%)$ & $79(94 \%)$ & $11(55 \%)$ & $1291(78 \%)$ \\
\hline Unfavorable $(G O S<4)$ & $65(25 \%)$ & $1(8 \%)$ & $5(6 \%)$ & $9(45 \%)$ & $373(22 \%)$ \\
\hline
\end{tabular}


Table 16. One year outcome for four patient groups admitted during 1980-2005 and treated both actively or conservatively.

\begin{tabular}{|c|c|c|c|c|}
\hline & \multirow{2}{*}{ Ruptured DACAAs } & \multicolumn{2}{|c|}{ Unruptured DACAAs } & \multirow{2}{*}{$\begin{array}{c}\text { All ruptured } \\
\text { aneurysms }\end{array}$} \\
\hline & & No SAH & Acute SAH & \\
\hline Number of patients & 277 & 94 & 56 & 2243 \\
\hline \multicolumn{5}{|l|}{ 1-year outcome } \\
\hline Good recovery & $159(57 \%)$ & $77(82 \%)$ & $29(52 \%)$ & $1196(53 \%)$ \\
\hline Moderate disability & $45(16 \%)$ & $12(13 \%)$ & $6(11 \%)$ & $352(16 \%)$ \\
\hline Severe disability & $34(12 \%)$ & $4(4 \%)$ & $4(7 \%)$ & $146(7 \%)$ \\
\hline Vegetative & $2(1 \%)$ & 0 & 0 & $4(0.1 \%)$ \\
\hline Dead & $37(13 \%)$ & $1(1 \%)$ & $17(30 \%)$ & $543(24 \%)$ \\
\hline \multicolumn{5}{|l|}{ Cause of death at 1-year } \\
\hline SAH related & $35(94 \%)$ & 0 & $14(82 \%)$ & $500(92 \%)$ \\
\hline Treatment related & $1(3 \%)$ & $1(100 \%)$ & 0 & $8(1 \%)$ \\
\hline Unrelated & $1(3 \%)$ & 0 & $3(18 \%)$ & $35(7 \%)$ \\
\hline Management mortality & $36(13 \%)$ & $1(1 \%)$ & $14(25 \%)$ & $500(22 \%)$ \\
\hline
\end{tabular}

\subsubsection{Outcome at one year}

Favorable outcome (GOS 4 or 5) at one year after primary SAH from a ruptured aneurysm was seen in $74 \%$ of the 277 DACA patients as compared to $69 \%$ of the 2243 patients with ruptured aneurysms of all sites in the Kuopio Cerebral Aneurysm Database (Table 16). The case-fatality rates at one year were $13 \%$ and $24 \%$, respectively, and most of the early deaths were directly related to $\mathrm{SAH}$ or its sequelae (Table 16).

\subsubsection{Factors predicting outcome at one year}

Risk factors predicting unfavorable outcome $(G O S<4)$ at one year follow-up for the ruptured DACA aneurysms, based on multivariate analysis, were the following: age, Hunt and Hess grade $\geq 3$ on admission, rebleeding before treatment, $\mathrm{ICH}, \mathrm{IVH}$, and severe preoperative hydrocephalus (Table 17). Other risk factors did not show statistical significance in the final multivariate model.

Table 17. Risk factors for unfavorable outcome (GOS < 4) at one year follow-up in 277 patients with ruptured DACA aneurysm during 1980-2005.

\begin{tabular}{|lcc|}
\hline Factor & $\begin{array}{c}\text { Univariate Analysis } \\
\text { OR (95\% CI) }\end{array}$ & $\begin{array}{c}\text { Multivariate Analysis } \\
\text { OR (95\% CI) }\end{array}$ \\
\hline Patient Age (per one year of age) & $1.04(1.02-1.07)^{\S}$ & $1.07(1.04-1.11)^{\S}$ \\
H\&H grade $\geq 3$ on admission & $6.46(3.34-12.52)^{\S}$ & $3.90(1.63-9.34)^{\S}$ \\
Re-bleeding before treatment & $1.59(0.83-3.03)^{\ddagger}$ & $3.72(1.50-9.24)^{\S}$ \\
ICH on pre-op CT & $4.91(2.60-9.31)^{\S}$ & $2.74(1.27-5.92)^{\S}$ \\
IVH on pre-op CT & $5.13(2.88-9.13)^{\S}$ & $2.89(1.36-6.13)^{\S}$ \\
Severe pre-op hydrocephalus & $13.52(4.78-38.24)^{\S}$ & $6.18(1.92-19.94)^{\S}$ \\
\hline
\end{tabular}

In multivariate analysis, odds ratios (ORs) were also adjusted for sex

$\S P \leq 0.01$

$\neq \mathrm{P}>0.05$ 
5.4.3. Unruptured DACA aneurysms 19802005 (publication II, part b)

\subsubsection{Unruptured DACA aneurysms without acute SAH}

Of the 94 patients with 104 unruptured DACA aneurysms, 57 had no history of prior $\mathrm{SAH}$ and 37 had SAH from another aneurysm followed by a good recovery. Of these 94 patients, 84 were treated with microsurgery and three were coiled. Seven patients were treated conservatively because of old age, small aneurysm size $(\leq 2 \mathrm{~mm})$ or some other reason. The aneurysm occlusion rate of $91 \%$ was the same as in the ruptured DACA aneurysms (Table 15). Intraoperative rupture of unruptured DACA aneurysm occurred in only six (7\%) patients, much less often than in patients with acute SAH (see below). There were 10 (12\%) patients with postoperative morbidity related to surgery, mostly transient neurological deficits. One patient (1\%) died due to postoperative hematoma and infarction. Favorable outcome (GOS 4 or 5 ) was observed in $95 \%$ of the 84 microsurgically treated patients (Table 15). There was no difference in outcome between patients with no history of $\mathrm{SAH}$ and those with a history of previous SAH.

\subsubsection{Unruptured DACA aneurysms with acute $\mathrm{SAH}$}

There were 56 patients presenting with unruptured DACA aneurysm(s) in conjunction with acute SAH from another aneurysm. Only $20(36 \%)$ of these 56 patients had their unruptured DACA aneurysms treated, all microneurosurgically in the same session as the ruptured aneurysm through additional craniotomy or exposure (Table 15). The two most important reasons for conservative treatment were small DACA aneurysm size $(\leq 2 \mathrm{~mm})$ in 16 patients and poor condition or death in 12 patients. Clipping of unruptured DACA aneurysms in SAH patients was associated with postoperative deficit in three $(15 \%)$ patients but with no mortality.
The incidence of intraoperative rupture was as high as 35\% among the 20 unruptured DACA aneurysms treated in patients with $\mathrm{SAH}$ from another aneurysm (see above). Outcome at one year follow-up was similar to outcome for other ruptured aneurysms according to Kuopio Cerebral Aneurysm Database (Table 16).

\subsection{Long-term follow-up of ruptured DACA aneu- rysms (publication III)}

\subsubsection{Rebleeding}

A new episode of SAH occurred in four (95\% $\mathrm{Cl} 1.09-10.2)$ of the 280 patients during the follow-up period: (1) rebleeding from a subtotally clipped DACA aneurysm at 17 years; (2) rupture of a de novo MCA aneurysm at two years; (3) rupture of an untreated MCA aneurysm at three years; and (4) rupture of a previously undiagnosed basilar bifurcation aneurysm at five years. The estimated cumulative risk for a new episode of $\mathrm{SAH}$ at 10 years was $1.4 \%$ $(95 \% \mathrm{Cl} 0.0-3.0 \%)$ and at 20 years $3.0 \%(95 \% \mathrm{Cl}$ 0.0-6.5\%). The incidence for recurrent SAH was 138 per 100 ooo follow-up years $(95 \% \mathrm{Cl} 38-$ $354)$, which indicates a risk ratio of $3.9(95 \% \mathrm{Cl}$ 1.5-7.3) compared to the general adult Finnish population with an annual incidence of $\mathrm{SAH}$ 35/100 000 [348].

\subsubsection{Mortality}

\subsubsection{Early mortality}

By the end of follow-up, 92 (33\%) of the 280 patients had died, including 36 patients who died within 12 months, implying a 13\% management mortality at one year. The causes of these 36 deaths were mostly due to the primary SAH in 33 (92\%) patients (Table 18 ). It is also 
noteworthy that 17 (47\%) of these 36 patients who died during the first 12 postoperative months were initially in Hunt and Hess groups 4 and 5 .

\subsubsection{Long-term mortality}

No patients died of recurrent SAH from a treated DACA aneurysm. One patient died due to SAH from a previously undiagnosed basilar bifurcation aneurysm. Of the 208 patients who had survived more than four years, 42 (20\%) died, with cardiovascular disease as death cause in $24 \%$ and cancer in $19 \%$. Of the 137 patients who survived for more than 10 years, 24 (18\%) died, with cardiovascular disease as death cause in $29 \%$ and cancer in $25 \%$ (Table 18 ).

\subsubsection{Excess mortality}

The 3-year cumulative relative survival ratio (CRSR) was $0.84(95 \% \mathrm{Cl} 0.78-0.88)$ implying $16 \%$ excess mortality for patients with ruptured DACA aneurysm during the first three years

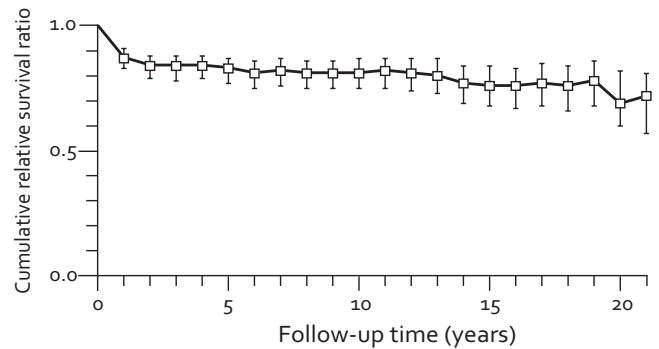

Fig. 17. Cumulative relative survival ratio (CRSR) of patients with ruptured DACA aneurysm $(n=280)$ as a function of follow-up time in years. Error bars indicate $95 \% \mathrm{Cl}$.

after diagnosis when compared with the general Finnish population (Fig. 17). At the fourth year of follow-up, the annual RSR attained 1.0 indicating no excess mortality thereafter (Fig. 18). A separate analysis of the patient group with good recovery at one year showed no excess mortality during the whole follow-up period. The three-year CRSR for women was 0.82 (95\% $0.74-0.87)$ and for men $0.86(95 \% \mathrm{Cl}$ $0.78-0.92$ ) indicating $18 \%$ and $14 \%$ excess

Table 18. Causes of death of the 92 patients who died during the long-term follow-up of 280 patients with ruptured DACA aneurysm.

\begin{tabular}{|c|c|c|c|c|c|c|}
\hline & \multirow{2}{*}{$\begin{array}{c}\text { Age at SAH } \\
\text { diagnosis } \\
\text { mean } \pm S D\end{array}$} & \multicolumn{4}{|c|}{ Time of death from diagnosis } & \multirow{2}{*}{$\begin{array}{c}\text { Total } \\
\mathbf{n}\end{array}$} \\
\hline & & $\begin{array}{c}<12 \text { mths } \\
n\end{array}$ & $\begin{array}{c}1-3 y r s \\
n\end{array}$ & $\begin{array}{c}\text { 4-10yrs } \\
n\end{array}$ & $\begin{array}{c}\text { >10yrs } \\
n\end{array}$ & \\
\hline Related to primary SAH & $54 \pm 14$ & 33 & 3 & 5 & 2 & 43 \\
\hline Re-bleeding from treated aneurysm & - & 0 & 0 & 0 & 0 & 0 \\
\hline $\mathrm{SAH}$ from another aneurysm & 46 & 0 & 0 & 1 & 0 & 1 \\
\hline Cerebrovascular & $58 \pm 12$ & 0 & 2 & 1 & 0 & 3 \\
\hline Cardiovascular & $52 \pm 11$ & 1 & 5 & 3 & 7 & 16 \\
\hline Cancer & $55 \pm 7$ & 0 & o & 2 & 6 & 8 \\
\hline Infection/Pneumonia & $58 \pm 11$ & 0 & 0 & 2 & 4 & 6 \\
\hline Trauma & $49 \pm 12$ & 1 & 1 & 1 & 1 & 4 \\
\hline Suicide & $48 \pm 18$ & 0 & 1 & 1 & 0 & 2 \\
\hline Other & $52 \pm 15$ & 1 & 2 & 2 & 3 & 8 \\
\hline Not known & 72 & 0 & 0 & 0 & 1 & 1 \\
\hline All deaths & $54 \pm 13$ & 36 & 14 & 18 & 24 & 92 \\
\hline
\end{tabular}

$\mathrm{n}=$ number of patients

$\mathrm{SD}=$ standard deviation 


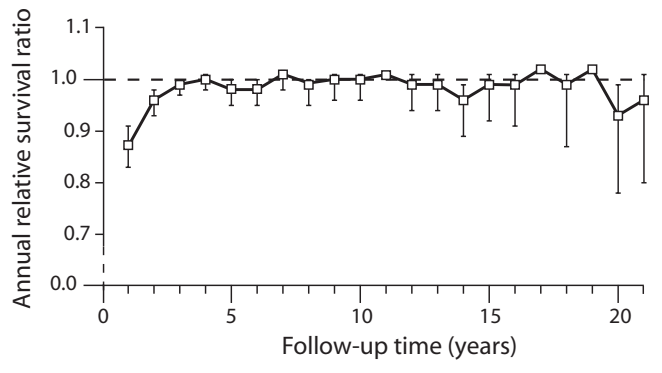

Fig. 18. Annual relative survival ratio of patients with ruptured DACA aneurysm $(n=280)$ as a function of follow-up time in years. Error bars indicate $95 \% \mathrm{Cl}$.

mortality during the first three years, respectively. Analysis by age at diagnosis ( $<45$ years, 45-60 years, $>60$ years) showed that the youngest group reached the annual $\mathrm{RSR}=1.0$ one to two years earlier with a lower excess mortality in general.

\subsection{Microneurosurgical technique for clipping DACA aneurysms (publi- cations IV-VI)}

\subsubsection{Approach}

The DACA aneurysms are generally approached through the anterior interhemispheric approach $[441,443]$. The only exceptions are some proximal $\mathrm{A} 2 \mathrm{As}$, which may require lateral supraorbital approach (LSO) or pterional approach (see section 5.6.12.1.), but here the vertical distance of $1.5 \mathrm{~cm}$ from the anterior skull base to the aneurysm is the limit. Through the interhemispheric approach the exposure depends on the course of the pericallosal arteries, the location of the aneurysm in relation to the GCC, projection of the dome, and possible ICH. For a right-handed neurosurgeon the rightsided approach is more convenient because both of the pericallosal arteries can be reached under the inferior margin of the falx for most of their course. A left-sided ICH or a left-sided associated anterior circulation aneurysm may require a left-sided approach. Importantly, the more proximal the DACA aneurysm lies, the more anterior the approach has to be planned (Fig. 19). With a wrong angle of approach the genu will obstruct the neurosurgeon's view towards the aneurysm base and prevent proper clip placement. We measure the position of the DACA aneurysm in relation to the outside cranium for the exact head positioning and bone flap placement. Usually the shortest route is selected. Some authors have used the bifrontal basal anterior interhemispheric approach $[32,390]$. We feel that the unilateral approach is less invasive and quicker, and provides equal exposure to the DACA aneurysms deep in the interhemispheric fissure. The technique of clipping ruptured or unruptured DACA aneurysms is almost the same. The only real difference is the lack of working space in acute SAH which makes the whole procedure more difficult. Neuronavigation may be of help in planning and executing the approach towards the aneurysm.

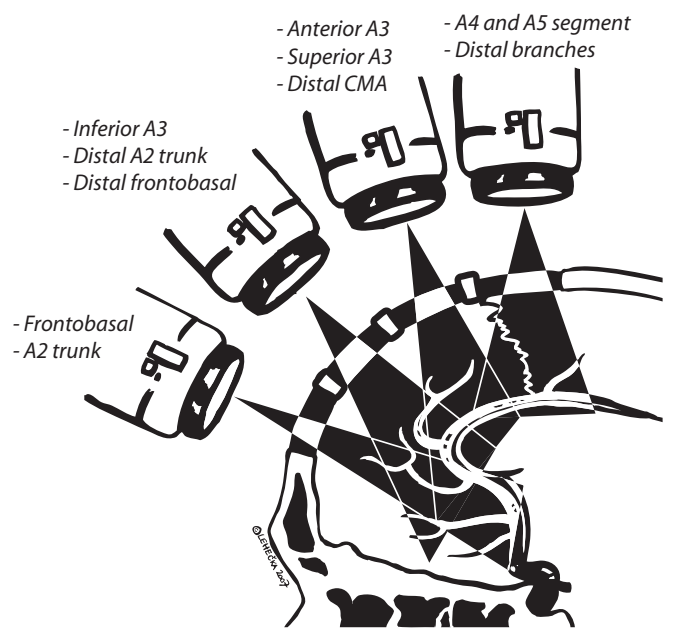

Fig. 19. Positioning of bone flap during interhemispheric approach for DACA aneurysms at different locations. 


\subsubsection{Positioning, craniotomy and dural opening}

For the anterior interhemispheric approach the patient is in supine position with the head fixed in a head frame and elevated about $20^{\circ}$ above the heart level. The head should be in neutral position with the nose pointing exactly upwards. Tilting the head to either side increases the chance that the bone flap is placed too laterally from the midline. This would make the entrance into the interhemispheric fissure and navigation more difficult. The head is slightly flexed or extended according to how proximal or distal the DACA aneurysm lies. In the optimal position the trajectory is almost vertical. If intraoperative DSA is considered, the frame pins should be placed accordingly or a carbon frame should be used for better visibility. It is our practice to adjust the position of the fixed head and body during the operation when needed [121].

After minimal shaving, an oblique skin incision with its base frontally is made just behind the hairline, over the midline, extending more to the side of the planned bone flap (see DACA - video 1). For some posterior approaches to AdistAs, a straight incision along the midline can be used. Location, curvature, and extent of skin incision depends on the hairline, dimensions of the frontal sinuses, and the orientation of the aneurysm. A one-layer skin flap is reflected frontally with spring hooks. Bicoronal skin incision is unnecessary since strong retraction with hooks often allows anterior enough exposure of the frontal bone. The bone flap is placed slightly over the midline to allow better retraction of the falx medially. The superior sagittal sinus may deviate laterally from the sagittal suture, more often to the right, and as far as $11 \mathrm{~mm}$ [410]. The size of the bone flap depends both on the surgeon's experience and on the presence of $\mathrm{ICH}$. We usually use a $3-4 \mathrm{~cm}$ diameter flap. Too small a flap may not provide sufficient space for working between the bridging veins. In most patients, only one burr hole in the midline over the superior sagittal sinus at the posterior border of the bone flap is needed. Through this hole, bone can be detached from the underlying dura. One has to be careful with the underlying sagittal sinus, particularly in the elderly with a very adherent dura. The bone flap is removed using a side-cutting drill. High speed drill can be used to smoothen the edges or to enlarge the opening if necessary. If the frontal sinuses are accidentally opened during the craniotomy, they should be packed and isolated with fat or muscle grafts and covered with pericranium.

The dura is opened under the operating microscope as a C-shaped flap with its base at the midline. The incision is first made in the lateral region and then extended towards the midline in the anterior and the posterior direction to prevent opening of the superior sagittal sinus. The dural opening should be planned so that possible meningeal sinuses and lacunae are left intact. Bridging veins may be attached to the dura for several centimeters along the midline. Careful dissection and mobilization of these veins is necessary. It is usually during the opening of the dura that unwelcome damage to the bridging veins takes place. Dural edges are elevated with multiple stitches extended over the craniotomy dressings to prevent epidural oozing into the surgical field.

If the neuronavigation system is used, the correct angle of the trajectory should be verified while planning the skin incision. With the bone flap removed and the dura still intact, the approach trajectory has to be checked again for correct working angle of the microscope. After the dura has been opened and CSF released, brain shift will make neuronavigation less reliable, and one becomes more dependent on the visible anatomic landmarks.

\subsubsection{CSF drainage}

In case of lateral supraorbital approach (LSO), we release CSF from the supracellar cisterns. In acute $\mathrm{SAH}$, the dissection is then continued from the supracellar cistern subfrontally 

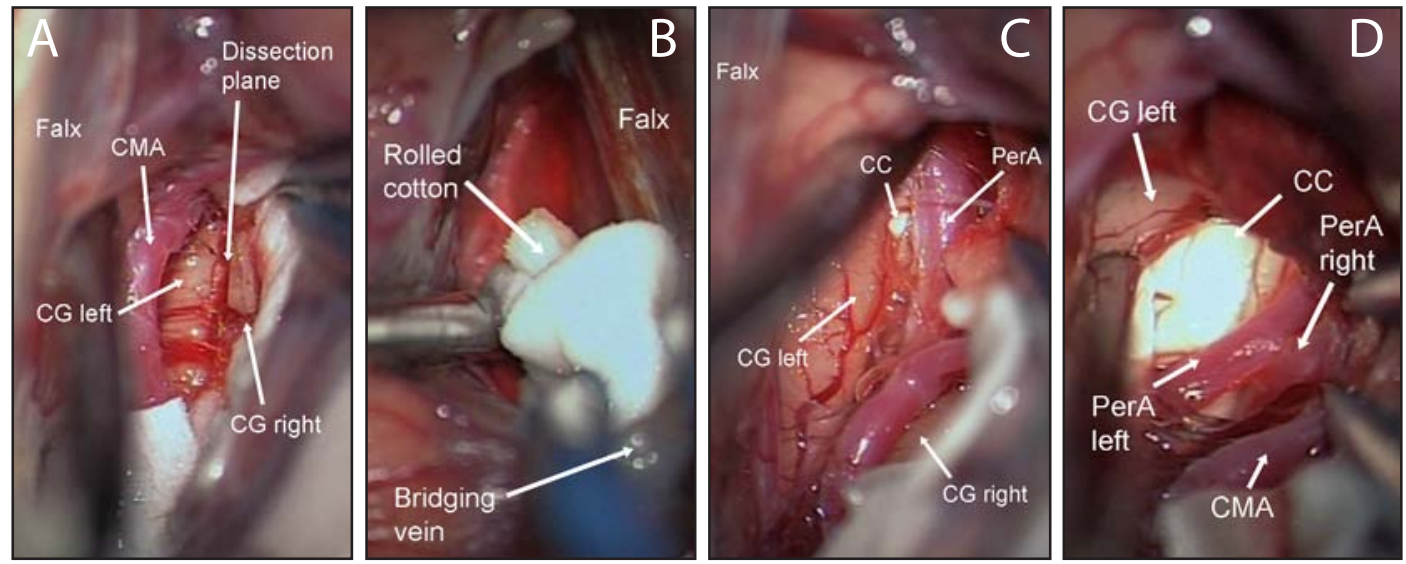

Fig. 20. Intraoperative photographs of the interhemispheric fissure with (A) callosomarginal artery $(C M A)$ and the tightly attached cingulate gyri (CG), (B) rolled cottons used as expanders, (C) pericallosal artery (PerA) in between the cingulate gyri, and (D) white color of the genu of the corpus callosum (CC) (see DACA — video 7).

towards the optic chiasm and the lamina terminalis which is opened for additional removal of CSF.

The interhemispheric approach provides less space as the callosal cistern is shallow and not much CSF can usually be removed. In unruptured DACA aneurysms, this space will nonetheless usually suffice. In acute SAH, CSF can be released by a puncture to the lateral ventricle at the lateral border of the craniotomy. As an alternative, the corpus callosum can be punctured with a closed bipolar forceps medial to the pericallosal artery, followed by opening of the forceps to create a small channel to the lateral ventricle for CSF release. Partial removal of the ICH may also provide enough space to be able to continue towards the aneurysm.

\subsubsection{Removal of ICH}

In case of a large $\mathrm{ICH}$ and possible lack of space (Fig. 10a-d), a small cortical incision is made accordingly and the hematoma is partially removed to start with to gain more space. This may cause rerupture of the aneurysm which would be difficult to control at this point. In removing the $\mathrm{ICH}$ clot, before or after clipping, only minor force should be applied not to sever the perforating arteries. $\mathrm{ICH}$ in the immediate vicinity of the aneurysm should be left in place until proximal and distal control have been obtained. In acute SAH, thick blood clots inside the interhemispheric fissure make dissection and visualization of the pericallosal artery and the aneurysm often difficult. Repeated irrigation with saline ("water dissection" [258]) and gentle suction can be used to flush the clots out and to provide better space for further dissection. Only the blood clots which obstruct the approach trajectory are removed. Too extensive removal can easily damage the surrounding brain tissue.

\subsubsection{Dissection towards the aneurysm}

For the interhemispheric approach, the images should be evaluated for several microsurgical aspects: depth of the falx; depth of the corpus callosum; depth and course of the pericallosal and the callosomarginal arteries; correct parent artery; location of the aneurysm with respect to the GCC (inferior, anterior, superior); size, orientation, and origin of the dome; and possible dislocations due to ICH. CTA should be carefully reviewed for calcifications in the arteries and the aneurysm wall. Calcified plaques in 

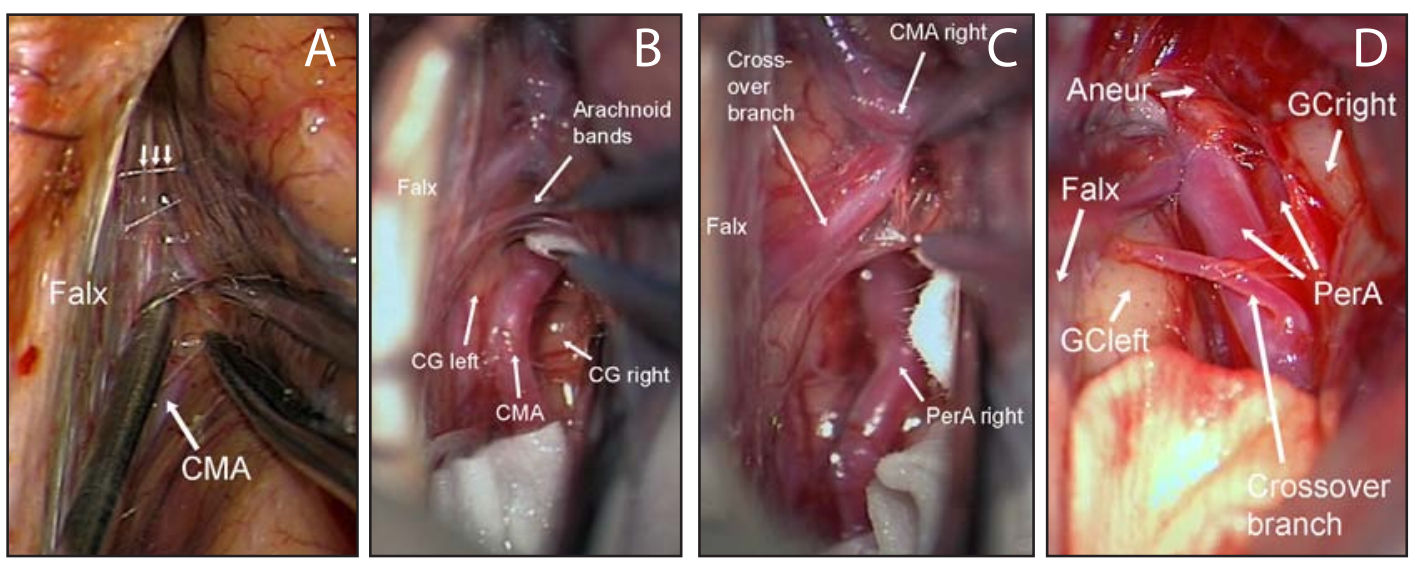

Fig. 21. Intraoperative photographs of the interhemispheric fissure showing (A) the arachnoid bands (small arrows) attached to the falx and the callosomarginal artery (CMA) in the cingulate sulcus, (B) the tightly attached cingulate gyri (CG left, CG right) and the CMA running on top of them, (C) a large crossover branch originating form the right CMA, and (D) pericallosal arteries (PerA) running parallel with ruptured AdistA surrounded by fresh blood clots and embedded in the right cingulate gyrus (CG right).

the parent artery will affect temporary clipping, and those at the dome risk intraoperative rupture and incomplete closure of the neck or even complete closure of the parent artery.

We use a hand held syringe to expose and to expand planes for further dissection, i.e., the water dissection technique of Toth [258]. Arachnoid membranes and strands are cut sharply by microscissors which can be also used as dissector when the tips are closed. Use of retractors is kept at minimum, and they are not routinely used at the beginning of the approach. Instead, bipolar forceps in the right hand and suction in the left, with cottonoids of different sizes as expanders, are used as microretractors [121]. When the interhemispheric fissure is widely opened and the frontal lobe mobilized, the retractor may be used to retain some space for clipping, but should otherwise be avoided. Rolled cottons, placed inside the interhemispheric fissure at the anterior and the posterior margin of the approach, provide a more gentle retraction than classical, mechanical retractors (Fig. 2ob).

Upon entering the interhemispheric fissure, bridging veins may obstruct the view, preventing even the slightest retraction of the frontal lobe. The veins are likely to restrict the working space and one may have to work between them. It may be of help to dissect some of them for a few centimeters from the brain surface. One may have to sacrifice a smaller vein, with the risk of venous infarction though. Extensive and long-lasting use of retractors, preventing the subsequent venous flow, may have the same result as severing the bridging veins.

Inside the interhemispheric fissure, after clearing the arachnoid adhesions (Fig. 21a), dissection is directed along the falx towards the corpus callosum (see DACA - video 4). The exact orientation of the approach trajectory depends on the location of the aneurysm with respect to the GCC. It is important to be aware of the microscope's angle and the exact head position. With a wrong angle of approach one gets easily lost inside the interhemispheric fissure with no good landmarks to guide towards the aneurysm. At the inferior border of the falx, the dissection plane is identified between the tightly attached cingulate gyri (Fig. 20a, 21b). The pericallosal artery may be found already in the cingulate sulcus, but in most cases the dissection must be continued deeper toward the corpus callosum (Fig. 20c), identified by 
its white color and parallel, transverse fibers (Fig. 2od). Falsely assuming the attached cingulate gyri as the corpus callosum, or other paired arteries as the pericallosal arteries, leads to serious problems in navigation.

Once inside the callosal cistern, both pericallosal arteries are visualized, often with a realization that they can be on either side of the midline. The artery leading to the aneurysm is identified and
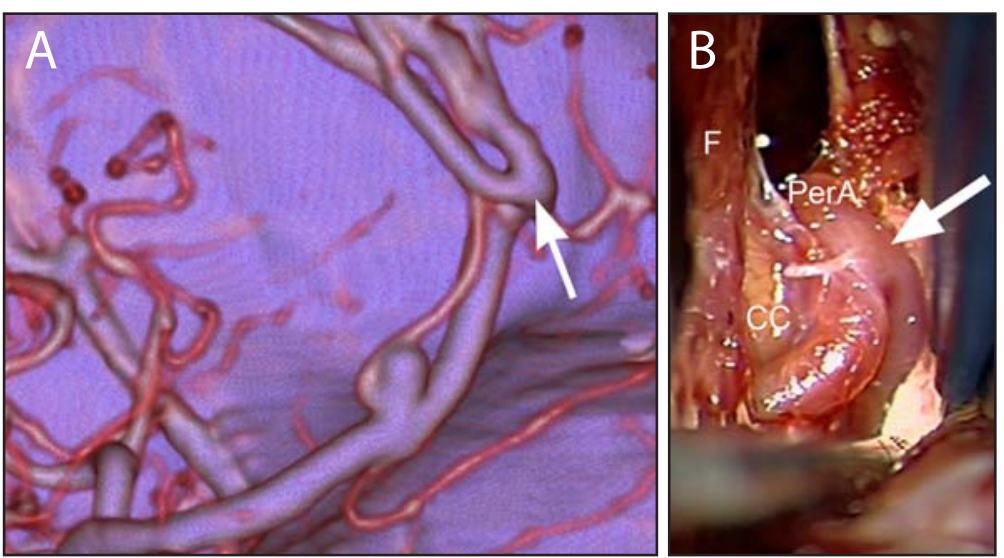

Fig. 23. (A) Azygos ACA with A2 trunk aneurysm as seen on CTA. (B) Intraoperative picture of the same patient during anterior interhemispheric approach showing the azygos ACA (PerA) with the bifurcation (arrow), the falx (F) and the corpus callosum (CC) (see DACA - video 4). followed to the proximal

direction towards the aneurysm. A tedious and careful dissection is performed in the deep and narrow proximal interhemispheric fissure (Fig. 22b). The aim is to identify the proximal part of the parent artery. Landmarks of help are the ori-
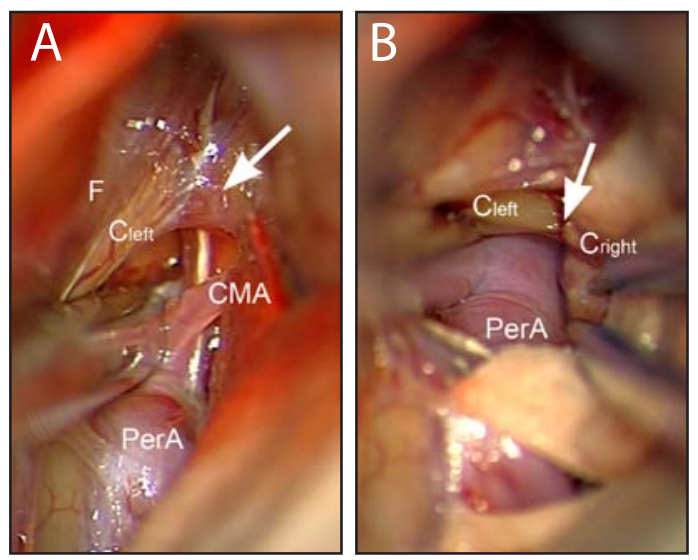

Fig. 22. Intraoperative photographs demonstrating the narrow interhemispheric space at the $(\boldsymbol{A})$ A3 segment, and (B) A2 segment of the ACA. The pericallosal artery (PerA) is running between the strongly attached cingulate gyri (C left, C right) below the lower margin of the falx (F) with dissection plane marked by arrows. The callosomarginal artery (CMA) originates from the $A 3$ segment. gin of the callosomarginal artery (Fig. 22a), the genu of the corpus callosum, and possible vascular anomalies recognized in the images (Fig. $23 a, b)$. Since the aneurysm is approached along the distal parent artery, the dome with its possible rupture site is likely to get in the way so as to hide the view to the proximal parent artery, i.e. the site for temporary clipping. Premature rupture at this phase, with little space and no proximal control, may cause great difficulties.

The dome often extends more to one side and is embedded in the pial layer of the cingulate gyrus (Fig. 21d). This may allow traversing along the opposite gyrus to get proximal control of the parent artery, which is often the most difficult part of the interhemispheric approach. The direction of the aneurysm may change due to retraction and blood may obscure the anatomy, making identification of the aneurysm difficult. Strong retraction is also likely to cause intraoperative rupture. We do not use partial callosal resection to enlarge exposure in the infracallosal region [54]. However, with appropriate head positioning and a well placed bone flap, it is possible to obtain adequate visualization of the aneurysm base without any callosal resection [181]. 


\subsubsection{Dissection of the aneurysm}

Small size, thin wall, and a relatively broad base involving branches make the dissection of the DACA aneurysm challenging in the narrow interhemispheric space. The proximal and distal parts of parent arteries as well as all the adjacent branches should be unhurriedly and painstakingly visualized before the final clipping (Fig. 21C). A small subpial resection is often necessary to allow the mobilization and visualization of the whole aneurysm dome.

In acute SAH the cingulate gyri are usually very tightly attached to each other (see DACA — video 9). Dissection of the proximal parent artery can cause great difficulties and it can easily damage both cingulate gyri (Fig. 21d). In such situations, at the expense of poor proximal control, the aneurysm is approached directly and a pilot clip is placed at the neck as soon as possible. With the pilot clip in place dissection of the dome is continued.

\subsubsection{Temporary clipping}

Temporary clipping facilitates sharp dissection of the aneurysm and the adjacent arteries. Dissection and preparation of the site for the temporary clip(s) should be performed with a blunt-tipped bipolar forceps or with a microdissector. One temporary clip, usually a small one, curved or straight, is applied proximal to the aneurysm (see DACA — video 8). The proximal clip can be close to the aneurysm but the distal ones should be at a distance not to interfere with the visualization and the permanent clipping of the aneurysm neck. In ruptured cases, CMA may require its own temporary clip. When the main part of the base is dissected, a short, straight pilot clip is applied and the temporary clip is removed. When removed, the temporary clip should be first opened carefully in place to test if any unwanted bleeding occurs. Removal in rush can be followed by heavy bleeding and great difficulties in replacing the clip. Furthermore, while removing the tempo- rary clip, even the slightest resistance should be noted as a possible involvement of a small branch in the clip or its applier. If access to the proximal parent artery cannot be achieved, direct pilot clipping is the only choice, and usually a small microclip is applied to the aneurysm base. Longer clips may involve or kink side branch(es).

\subsubsection{Clipping of the aneurysm}

A proper selection of clips with different shapes and lengths of blades and applicators suiting the imaging anatomy of the aneurysm should be ready for use. Additionally, a limited selection of final clips is needed for temporary clipping of the arteries and bipolar reshaping of the aneurysm dome. DACA aneurysms are generally small with many surrounding small branches. To prevent kinking or occlusion of adjacent branches, the smallest but adequate final clip should be selected. If bipolar reshaping is not considered, the blade of a single occluding clip should be 1.5 times the width of the base. We prefer inserting first a pilot clip to the aneurysm dome, preferring Sugita clips for their wide opening distance and blunt tips. The pilot clip is later changed for a smaller and lighter final clip, after reshaping of the dome by bipolar coagulation (see DACA - video 6). Adequate dissection, proper clip sizes, and painstaking checking that the clip blades are well placed up to their tips are required to preserve the adjacent branches. If the whole dome is dissected free, it may be dislocated to other side with suction to allow better position for the final clip (see DACA - video 4). If the first clip slides, exposing some of the neck, another clip may be applied proximal to the first one for final closure ('double clipping'). Removal of the retractors and cottonoids may lead to kinking of the parent artery or compression of the perforators by the clip blades or the clip itself. The flow has to be checked once more and papaverine applied to release or prevent local vasospasm with only a transient effect. 


\subsubsection{Intraoperative aneurysm rupture}

The DACA aneurysm may rupture while the neurosurgeon is entering the interhemispheric fissure or dissecting the aneurysm base. The risk is high, since most of the aneurysms are oriented so that their dome is encountered before visualization of the proximal artery. The $A_{2} A s$ and inferior $\mathrm{A}_{3} \mathrm{As}$ are particularly problematic and achieving proximal control may be difficult (Fig. 22b). Control should be first attempted via suction and compressing the bleeding site with cottonoids. We keep a second suction prepared before clipping. Sudden and short hypotension by cardiac arrest, induced by i.v. adenosine, can be used to facilitate quick dissection and application of a pilot clip in case of uncontrolled bleeding [321]. A pilot clip may be inserted to a ruptured secondary pouch if visible. Otherwise, temporary clips are inserted proximally on the parent artery, possibly also on branches such as CMA, to allow dissection of the base and final clipping. A small and thin walled aneurysm may rupture at its neck during dissection. In such a case, reconstruction of the base involving a part of the parent artery in the clip should be attempted under temporary clipping.

\subsubsection{Verification of clipping}

We routinely use micro-Doppler and intraoperative, noninvasive, indocyanine green (ICG) infrared angiography to check the patency of the proximal and distal arteries and branches [315,316], but unexpected occlusions are sometimes seen in postoperative angiography even in very experienced hands [191]. The ICG helps the orientation during dissection, and it visualizes wall thickness and plaques, perforating arteries, and incomplete neck occlusion. ICG angiography reduces the need for invasive intraoperative angiography for clipping control, but DSA is still required in giant and complex aneurysms. The quality and reliability of postoperative CTA is better when titanium clips are used.

\subsubsection{Associated aneurysms}

DACA aneurysms are often associated with other aneurysms, in 50\% of cases in our series. Associated DACA aneurysms were seen in 7\% of the patients. Most DACA aneurysms can be reached under the falx even if they are on the contralateral side of the craniotomy. Therefore, bilateral interhemispheric approach is not necessary. Our strategy is to clip all aneurysms that can be exposed through the same approach, i.e. only DACA aneurysms in case of interhemispheric approach. This may not be advisable if clipping of the ruptured aneurysm is difficult or the brain is swollen due to acute $\mathrm{SAH}[52,330]$. Also, this technique of clipping multiple aneurysms simultaneously at different locations is not recommended during the early learning curve. In acute $\mathrm{SAH}$, if there are problems in clipping the ruptured aneurysm, one should not continue with the unruptured one(s). We do not recommend multiple craniotomies for ruptured cases in the acute phase.

\subsubsection{Special considerations for each DACA aneurysm location}

\subsubsection{Frontobasal A2As (publication IV)}

A2As located on the frontobasal branches of the $A 2$ segment are very rare (Fig. 24a,b). They are usually saccular and small. We approach them through the LSO or the interhemispheric approach, depending on their distance from the anterior fossa.

When close to the skull base, we use the LSO with the benefit of more familiar anatomic landmarks. The vertical distance of $1.5 \mathrm{~cm}$ from the anterior skull base to the aneurysm is the limit. The LSO craniotomy is a more subfrontal and less invasive modification of the pterional approach for the anterior circulation aneurysms (see DACA - video 2)[120]. For the LSO approach, it is important to evaluate the distance between the ACoA complex and the optic chiasm, the courses and lengths of the $A_{1}$ 

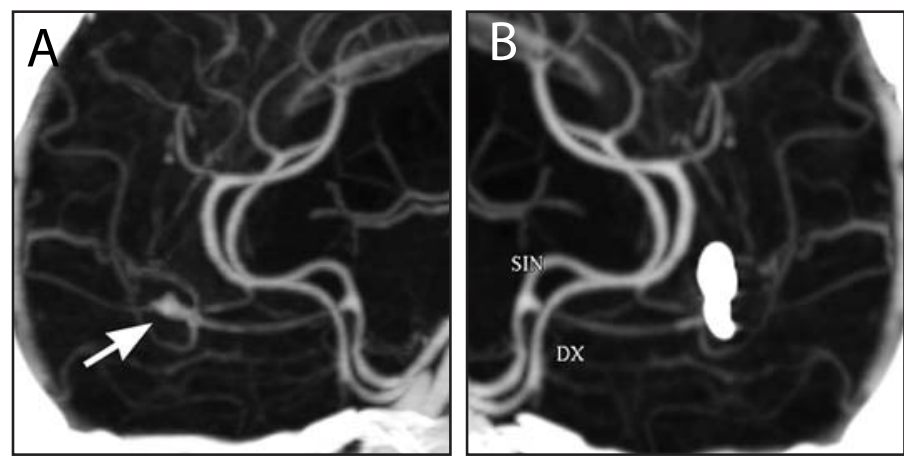

Fig. 24. Unruptured frontobasal $A 2 A$ (arrow) at the distal frontopolar artery as seen on $(\boldsymbol{A})$ preoperative and $(\boldsymbol{B})$ postoperative CTA (see DACA—video 5).

segments, the number and orientation of the $A_{2}$ segments, the distance of the $A_{2} A$ from the $A_{1}-A_{2}$ junction, and also the branches in the vicinity of the aneurysm. A small frontobasal resection of the rectal gyrus is usually needed to find the aneurysm. The extent of gyrus rectus resection depends on the distance between the ACoA and the optic chiasm, which can be measured from the images. The RAH and the perforators entering the optic chiasm should not be damaged. The goal is to identify both A1s, the ACoA, and the origins of both A2s.

If the interhemispheric approach is used, the angle of the approach and the distance of the aneurysms from the cranial vault should be evaluated on the images (see DACA — video 5).
A small, straight final clip is usually needed to exclude these aneurysms from the circulation. A neuronavigation system may be of help in finding these aneurysms, but brain shift after CSF release and frontal lobe retraction may be confounding.

\subsubsection{A2 trunk A2As (publica-} tion IV)

A2 trunk aneurysms on the proximal $\mathrm{A}_{2}$ segment can be reached either through the LSO (see DACA - video 3), or the anterior interhemispheric approach. The approach is chosen according to the size and orientation of the aneurysm, its vertical distance from the anterior skull base, possible vascular anomalies, previous surgeries (arachnoid adhesions), rupture status of the aneurysm, projection of the dome, and also the surgeon's preferences. In acute $\mathrm{SAH}$, we prefer the LSO because of (a) the possibility of fenestrating the lamina terminalis, (b) proximal control of the ACoA complex, and (c) easier orientation. The interhemispheric approach, lacking good anatomic landmarks, becomes even more difficult in acute $\mathrm{SAH}$ with a swollen brain and also blood clots obstructing the view. A catheter inserted through the fenestration in the lamina terminalis into the
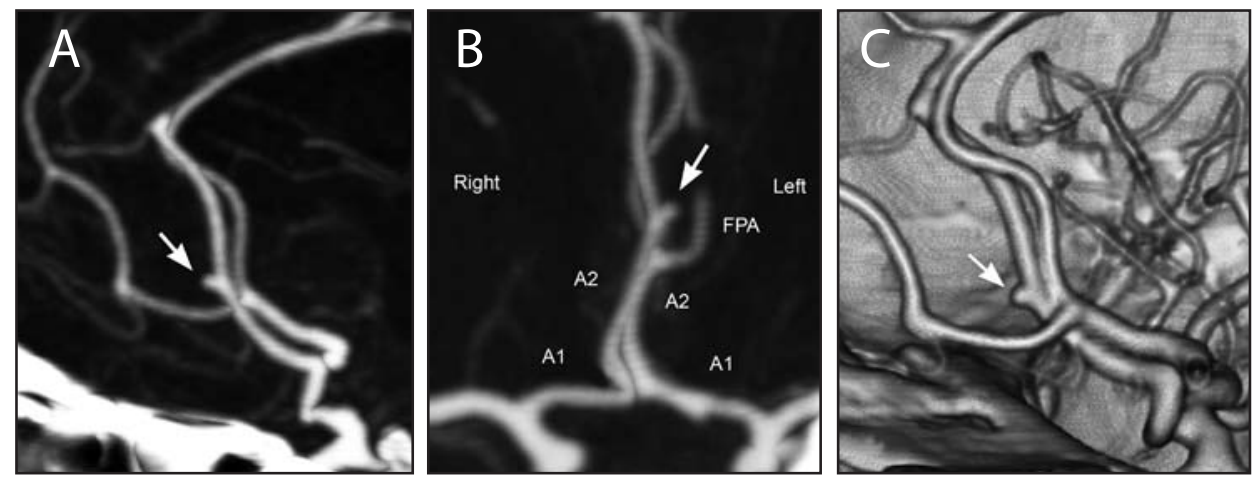

Fig. 25. CTA images of small, unruptured $A 2$ trunk aneurysm (arrow) as seen on (A) sagittal view, (B) axial view, (C) $3 D$-reconstruction. 
third ventricle can be left for ICP monitoring and further CSF removal postoperatively.

The anterior interhemispheric approach is used for more distally located $A 2$ trunk aneurysms (Fig. 25a-c) (see DACA — video 4). The gap between the cingulate gyri is deep and narrow, with possible strong adhesions (Fig. 22b). Blood clots and brain swelling in acute SAH make this approach even more demanding. The exposure of the pericallosal artery and the aneurysm depends on the course of the vessel, its location in relation to the corpus callosum, projection of the dome, and an associated $\mathrm{ICH}$.

The main difficulty is to localize the A2 trunk aneurysm, regardless of the approach.
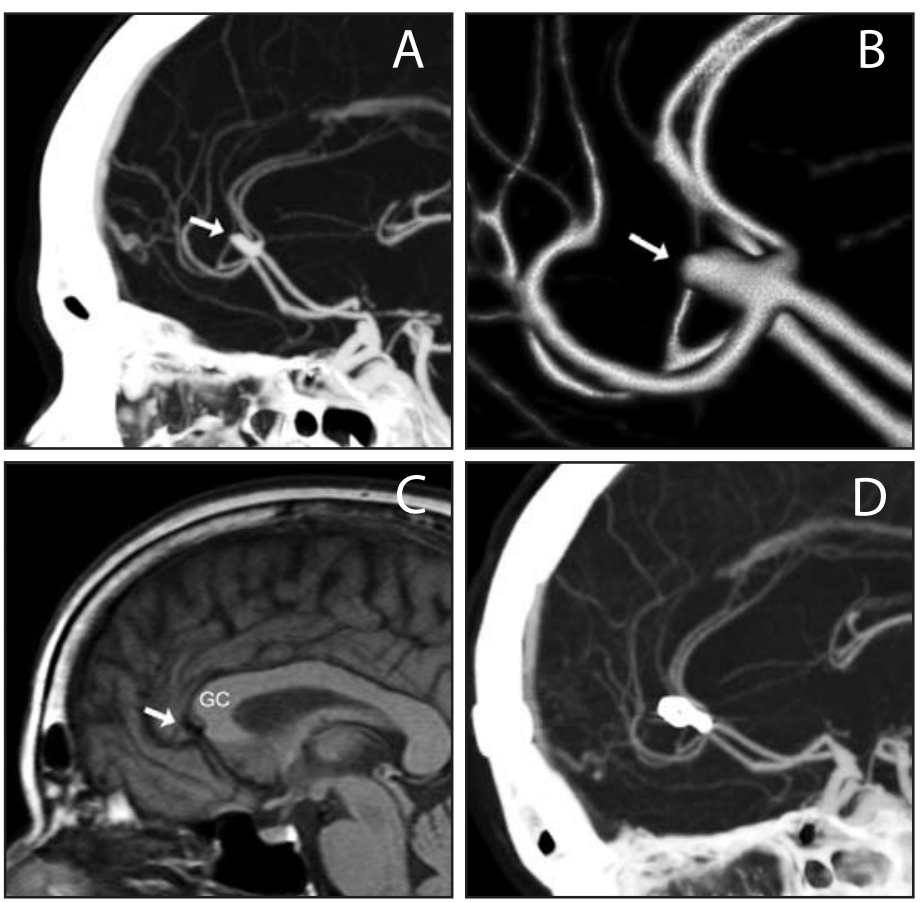

Fig. 26. Unruptured inferior $A 3 A$ (arrow) as seen on (A) preoperative $2 D C T A,(B)$ preoperative $3 D C T A,(C)$ preoperative $M R l$, and (D) postoperative CTA (see DACA - video 6). Intraoperative DSA may improve navigation during the interhemispheric approach, but in the LSO, the oblique head position is a limiting factor. The neuronavigation system may help early navigation before CSF removal and frontal lobe mobilization.

Proximal control is particularly difficult in this aneurysm location especially through the interhemispheric approach (see DACA — video 4). A small, straight final clip is usually needed to exclude these aneurysms from the circulation. Extreme care should be taken not to involve the $\mathrm{RAH}$ in the clip. Distal A2 trunk aneurysms are usually well above the origin of the RAH.

\subsubsection{Inferior $A_{3} A s$ (publication V)}

All $A_{3} A s$ are approached via the interhemispheric approach. The inferior $A_{3} A$ s are located at the junction of the $A_{2}$ and $A 3$ segments, inferior to the GCC (Fig. 26a-d)(see DACA — video 6 , and DACA - video 7). They require more

anterior approach than the other $\mathrm{A}_{3} \mathrm{As}$ so that the genu does not obstruct the view towards the base (Fig. 19). Inferior A3As usually point forward and slightly upward, with a possible deviation to either side. Proximal control is particularly difficult to obtain in the inferior $\mathrm{A}_{3} \mathrm{As}$. Due to their deep location and poor proximal control, inferior $\mathrm{A}_{3} \mathrm{As}$ are usually more difficult to clip than anterior or superior $A_{3} A$ s. As a general rule, the more proximal the $A_{3} A$ lies, the more difficult the clipping is going to be as one has to work deeper in a narrower gap.

The bone flap should be placed as anteriorly as possible without opening the frontal sinuses. Inside the interhemispheric fissure, the dissection is directed along the falx towards the anterior margin of the GCC which is the first landmark. The corpus callosum and both A3s are identified and followed along the curved surface of the genu towards the $\mathrm{A}_{2}-\mathrm{A}_{3}$ junction where the $\mathrm{A}_{3} \mathrm{~A}$ lies. 


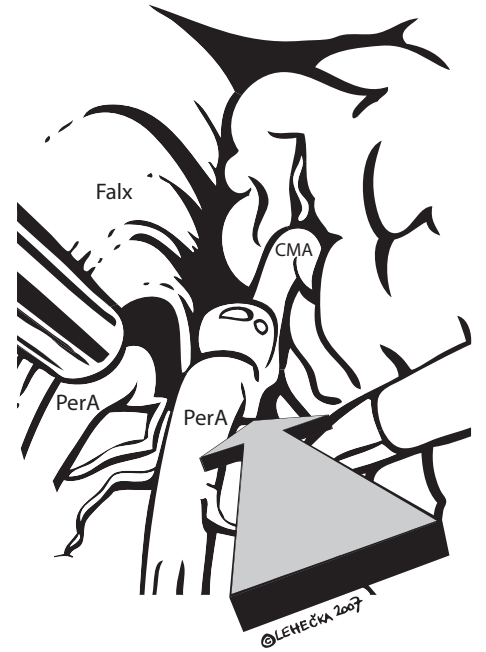

Fig. 27. Proximal control of the parent pericallosal artery (PerA) can be obtained from below the $\mathrm{A}_{3} \mathrm{~A}$.

The parent artery should be visualized if possible, without damaging the cingulate gyri. This may be easier from behind the $A_{3} A$ than in front of it (Fig. 27). If proximal control cannot be obtained, a pilot clip is placed at the dome and under its control the final dissection is carried out. The pilot clip is exchanged for the smallest possible final clip. Care is taken not to occlude branch(es) at the aneurysm base.

\subsubsection{Anterior $\mathrm{A}_{3}$ As (publication V)}

The anterior $\mathrm{A}_{3} \mathrm{As}$ are located at the mid section of the $A 3$ segment, anterior to the GCC (Fig. 28a-d)(see DACA - video 8, and DACA — video 9). The genu does not obstruct the base of the anterior $A_{3} A s$ as much as in the inferior $\mathrm{A}_{3} \mathrm{As}$, so it is somewhat easier to obtain proximal control. The anterior $\mathrm{A}_{3} \mathrm{As}$ are usually oriented forward and upward.

A paramedian craniotomy should be placed more posteriorly than for the inferior $\mathrm{A}_{3} \mathrm{As}$ (Fig. 19). The approach angle should point directly towards the aneurysm. Dissection in the interhemispheric fissure is first directed towards the GCC and, after both A3s have been identified, the appropriate $A_{3}$ is followed in the proximal direction. The dissection is directed around the aneurysm dome and, if possible, the proximal $A_{3}$ is exposed.

Final, sharp dissection of the base and the dome is then performed, if possible, with the help of temporary clips. The final clip should be the smallest possible to prevent kinking.

\subsubsection{Superior $A 3 A s$ (publication V)}

The superior $A_{3} A s$, the least frequent of all $\mathrm{A}_{3} \mathrm{As}$, are located at the distal part of the $\mathrm{A}_{3}$ segment, superior to the GCC (Fig. 29a-d). The genu does not obstruct the approach trajectory and the parent proximal $\mathrm{A}_{3}$ can be usually visualized for temporary control. Superior $\mathrm{A}_{3} \mathrm{As}$ are often directed upwards, even backwards.

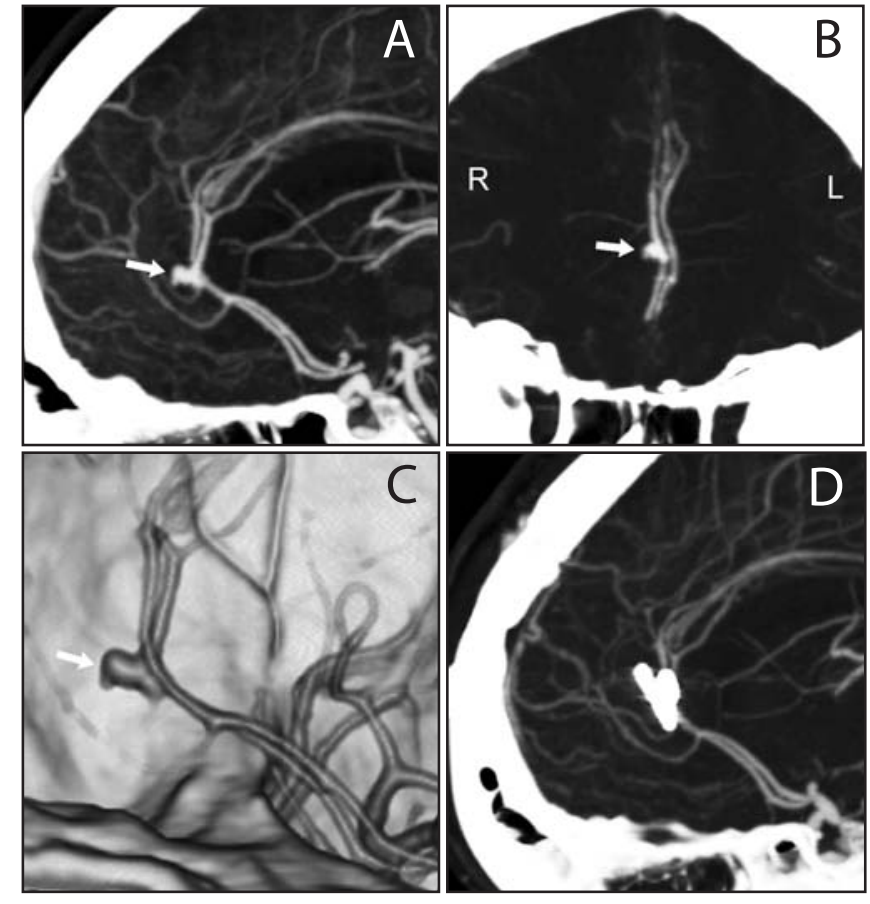

Fig. 28. Ruptured anterior $A 3 A$ (arrow) as seen on (A) preoperative sagittal CTA, $(\boldsymbol{B})$ preoperative coronal CTA, $(\boldsymbol{C})$ preoperative $3 D C T A$, and $(\boldsymbol{D})$ postoperative CTA. 


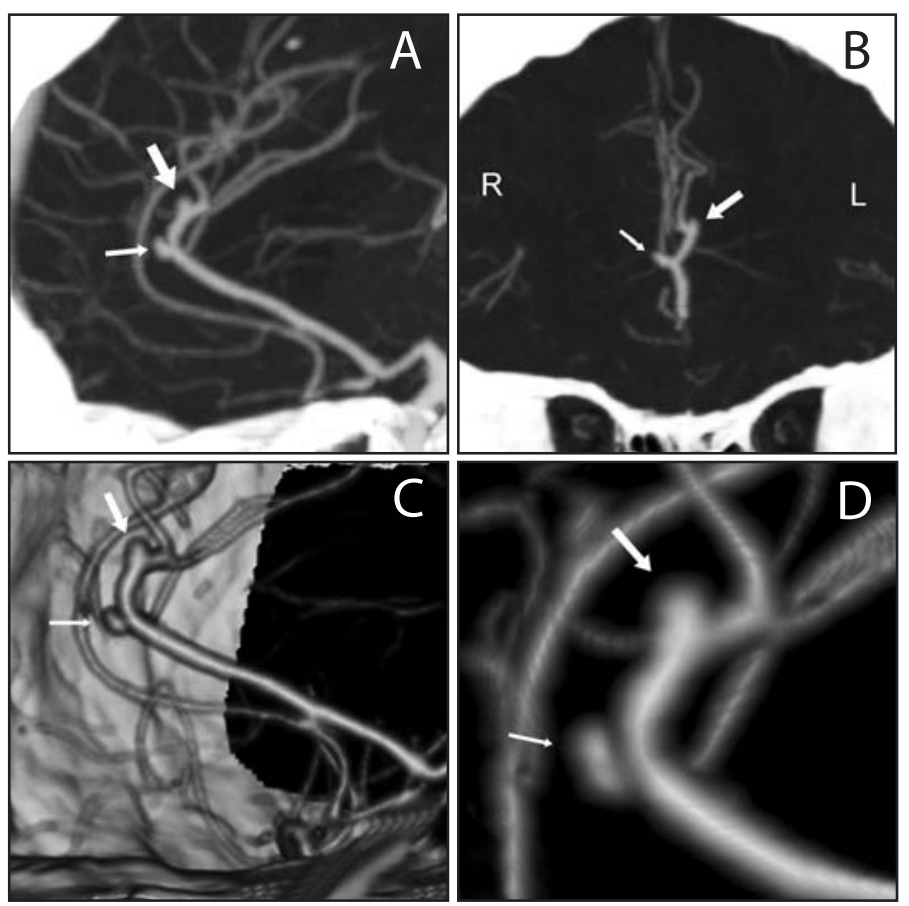

Fig. 29. Unruptured anterior $A 3 A$ (small arrow) and unruptured superior $A_{3} A$ found on the same pericallosal artery as seen on CTA in (A) sagittal view, (B) coronal view, (C and $\boldsymbol{D}) 3 D$ reconstructions.

The craniotomy is almost the same as in the anterior $\mathrm{A}_{3} \mathrm{As}$. The approach angle should point directly towards the aneurysm.

Dissection in the interhemispheric fissure is directed towards the corpus callosum, which is identified along with both $\mathrm{A} 3 \mathrm{~s}$ and followed proximally. In superior $\mathrm{A}_{3} \mathrm{As}$, it is possible to arrive almost directly at the base of the aneurysm. Exposure of the parent $A_{3}$ should be easier than in more proximal $\mathrm{A}_{3} \mathrm{As}$. Dissection of the aneurysm is continued under proximal control. The final clip should be as short and light as possible to prevent kinking of the arteries.

\subsubsection{A4 and $A_{5}$ aneurysms (publication VI)}

All the AdistAs are approached via the interhemispheric approach, the correct site of the exposure depending on the anterior-posterior location of the AdistA (see DACA — video 10, and DACA - video 11). The approach for $\mathrm{A}_{4}$ and $\mathrm{A}_{5}$ aneurysms is tailored according to the relation of the aneurysm to the corpus callosum and the lower margin of the falx, course of the pericallosal arteries, projection of the dome, and presence and size of a possible ICH (Fig. $30 a-c)$. The $A_{4}$ segment and the proximal $A_{5}$
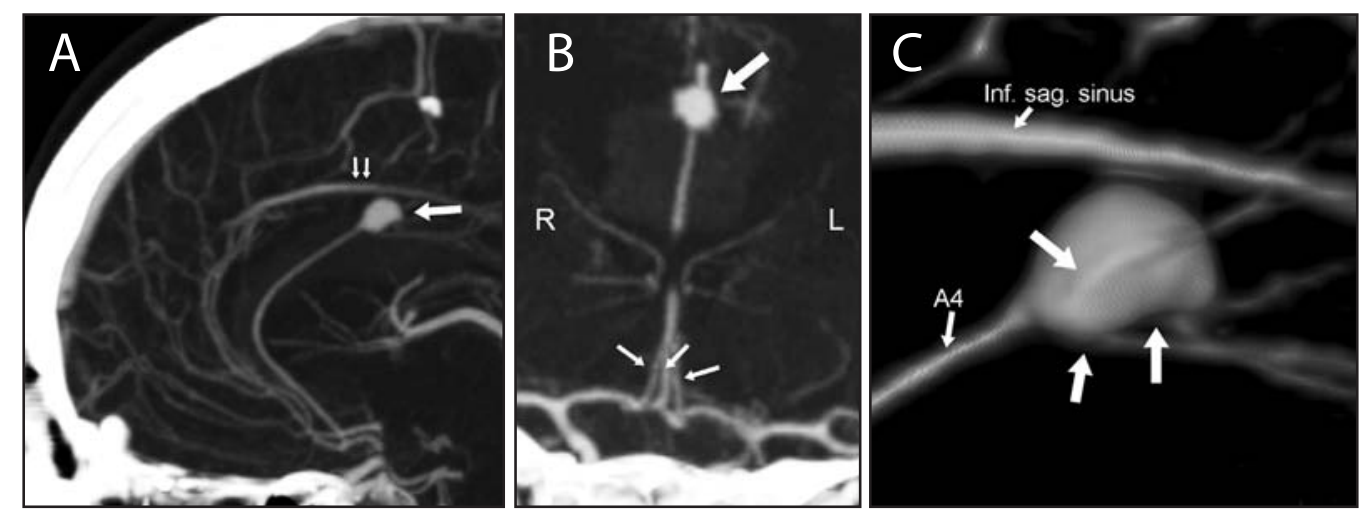

Fig. 30. Ruptured A4 segment AdistA (large arrow) presenting with ICH (A) located below the free margin of the falx and the inferior sagittal sinus (small arrows). Patient has (B) triplicated ACA (arrows) with the AdistA on the middle ACA with (C) three branches originating from the base (arrows) (see $D A C A$ - video 10). 
segment usually lie below the inferior margin of the falx (see DACA - video 10), but the distal A5 segment may be located above it requiring the bone flap on the same side as the aneurysm (see DACA — video 11).

We measure the distance of the aneurysm from the outside cranium, and usually choose the shortest approach. The coronal suture is an excellent intraoperative landmark that should always be identified in the preoperative CT and MRI scans. $A_{4}$ and $A_{5}$ aneurysms are very difficult to locate intraoperatively as there are no consistent anatomic landmarks in their vicinity for the orientation inside the interhemispheric fissure. Unlike in the $\mathrm{A}_{2} \mathrm{As}$ and the $\mathrm{A}_{3} \mathrm{As}$, the GCC can no longer be used as an anatomic landmark for orientation. Consequently, the neuronavigator is often helpful in finding the correct angle and approach to the aneurysm.

In AdistAs, distal temporary clipping is seldom needed due to the small caliber of the distal branches. The shortest and lightest possible final clip is used.
5.6.12.7. Distal branch AdistAs (publication VI)

Distal branch AdistAs are located almost always above the free margin of the falx (Fig. 31a-e), which requires the bone flap to be on the same side as the aneurysm, unless the lower portion of the falx is transected. As with $\mathrm{A}_{4}$ and $A_{5}$ aneurysms, selecting the appropriate approach angle is very difficult and intraoperative identification of the aneurysm may cause great problems due to the lack of proper anatomic landmarks. The neuronavigation system is highly recommended, because these aneurysms are rare and only a few neurosurgeons are used to dealing with them. With a wrong angle of approach one easily gets lost inside the interhemispheric fissure with no good landmarks to guide towards the aneurysm (see DACA - video 12). If possible, we try to approach the distal branch aneurysms along their proximal parent artery to obtain proximal control early in the surgery. Again the shortest and lightest final clip is used.
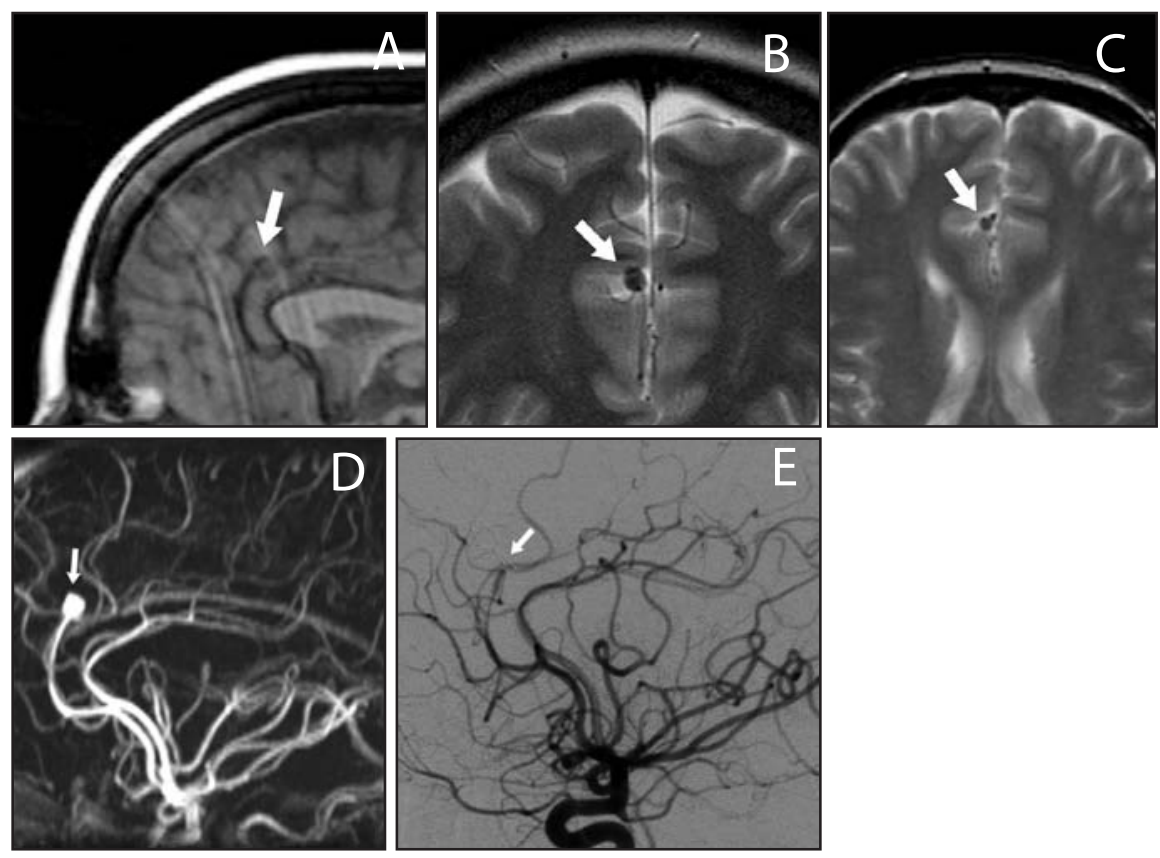

Fig. 31. Unruptured distal CMA aneurysm (arrow) as seen on preoperative (A) sagittal, (B) coronal, and (C) axial MRI images with the aneurysm in the cingulate sulcus. (D) Preoperative MRA and (E) postoperative DSA show aneurysm location at the bifurcation of the distal CMA. 


\section{Discussion}

This series of 517 DACA aneurysms is by far the largest published to date. It is a consecutive series of all DACA aneurysms treated at two Finnish institutions responsible for both primary and secondary care of all SAH patients in their catchment areas. The data are based on an ethnically homogenous population with good medical records and complete follow-up of all patients. The large sample size supports multivariate analysis of prognostic factors. The median follow-up of 10 years provides important data on rebleeding and mortality rates for ruptured and treated DACA aneurysms. Finally, we were able to compare ruptured DACA aneurysms to ruptured aneurysms in other locations in patients with similar characteristics and treatment selection. Results from this study are important when planning microneurosurgical or endovascular treatment of DACA aneurysms.

\subsection{Anatomic features}

\subsubsection{DACA aneurysm locations}

Most DACA aneurysms, $69-82 \%$ of cases, are found on the $A 3$ segment of the ACA, sometimes called the pericallosal artery-callosomarginal artery junction or "loco classico" [52,122, $142,279,312,383,449]$. We prefer the term $A_{3}$ aneurysms, or $A_{3} A s$, since in the present series the CMA was present at the base of these aneurysms in only $84 \%$ of the patients. In our material, $84 \%$ of all the DACA aneurysms were located at the $A 3$ segment. Of these, $78 \%$ were located anterior, $21 \%$ inferior, and only $1 \%$ superior to the GCC, an important division for microsurgical treatment. The angle of approach has to be selected based on this relationship of the aneurysm with the genu, since with a wrong angle of approach the GCC will obstruct the neurosurgeon's view towards the aneurysm base and prevent proper clipping. For endovascular treatment the distally located aneurysms will be difficult to reach via the small diameter parent arteries and there will be a lack of stability and support for optimal coil deployment.

\subsubsection{Aneurysm size and dome orientation}

DACA aneurysms are small with the usual size of 5-8 mm [32,52,122,245,264,279,383], in our series $6 \mathrm{~mm}$. Notably, $64 \%$ of all the DACA aneurysms and $51 \%$ of all the ruptured ones were smaller than $7 \mathrm{~mm}$. Giant DACA aneurysms are extremely rare. The statistical analysis showed no correlation between the size of the ruptured DACA aneurysm and the outcome at one year. Based on our results, at least in the Finnish population with high incidence of $\mathrm{SAH}$ [84], the occlusive treatment of unruptured DACA aneurysms smaller than $7 \mathrm{~mm}$ is justifiable. For endovascular treatment, the small size of DACA aneurysms, especially those smaller than $3 \mathrm{~mm}$ (20\% in our material), will make proper coil placement difficult or sometimes impossible.

Most of the DACA aneurysms (93\% in our series) have their domes oriented either forwards or upwards. For clipping through the interhemispheric approach this poses a problem, as the dome will initially obstruct the view towards the parent artery. Consequently, controlling the parent artery becomes challenging, especially in cases of intraoperative rupture.

\subsubsection{Aneurysm base and associated branches}

DACA aneurysms have been observed to have broad bases with possible calcifications and originating branches [122,443]. Our data proved these observations. In $81 \%$ of the aneurysms, the neck-to-dome ratio was more than 
$1: 2$, with $25 \%$ having their neck width equal to the dome width (1:1). At the same time, the neck was wider than the parent artery in $68 \%$ of the aneurysms. We identified at least one arterial branch at the base in $94 \%$ of the aneurysms. This incidence was even higher for the $A_{3}$ segment where $99 \%$ of aneurysms had branch(es) at their base. All these findings suggest that, irrespective of the treatment modality, it will be challenging to occlude DACA aneurysm completely without compromising the patency of either the parent artery or one of the originating branches. Therefore, clips should be as short as possible, often curved, to prevent accidental occlusion of one of the branches and to avoid kinking of the narrow parent artery. Reshaping of the aneurysm dome with bipolar coagulation before final clipping may sometimes be necessary. Coils need to be well stabilized inside the aneurysm dome to prevent them from migrating into the base and the originating branches.

\subsubsection{ACA anomalies}

In our series, $23 \%$ of the patients had anomalies of the ACA. Bihemispheric ACA, the most frequent one, was seen in $15 \%$ of our patients. Azygos ACAs or triplications of ACA were both present in $4 \%$. The azygos ACA was less frequent than what we had expected based on previous reports $[32,142,205,383,443]$. This is mainly due to the modern DSA and CTA imaging used in this study which allowed better distinction between the different types of anomalies. It is actually very difficult to distinguish a true azygos ACA from the bihemispheric ACA on normal DSA, even with compression of the contralateral carotid artery $[14,176]$. In patients with triplicated ACA, the aneurysm was found in all cases on the middle artery running along the corpus callosum, also called the hemispheric type of median callosal artery [415]. The only anomalies which could not be clearly observed from our CTA or DSA data were the small crossover branches from the distal pericallosal artery to the contralateral hemisphere, which should be present in $26-64 \%$ of patients [294,380]. This would have required a selective contrast injection into each A2. However, these crossover branches were frequently observed during surgery (Fig. 21C,d).

Complex bi-, tri-, and quadrifurcations arising from the distal anomalous ACA are thought to contribute to increased blood flow and shear stress on the vessel wall, increasing the susceptibility to aneurysm formation at these locations $[10,266]$. Of our patients with ACA anomalies, $96 \%$ presented the DACA aneurysm on the dominant pericallosal artery. The most typical location was at the bifurcation of this artery in $77 \%$. There were usually two to four branches originating from the base of such an aneurysm and they supplied both hemispheres (Fig. 15ac). Therefore, in patients with ACA anomalies, damage to the parent artery or occlusion of the branches at the neck will likely result in large, bilateral ischemic areas.

\subsubsection{Associated aneurysms and AVMs}

A high incidence of associated aneurysms in patients with DACA aneurysms has been reported previously, in $25 \%$ to $55 \%$ of the patients $[52,122,142,279,312,368,376,383,438$, 443] . In our series, $52 \%$ of the patients had associated aneurysms, much more than the 28-35\% reported for other aneurysm locations $[49,50,70,140,331]$. Statistical analysis in our series did not show any correlation between multiple aneurysms and hypertension, sex or polycystic kidney disease. High incidence of associated aneurysms necessitates careful decision making on how to treat the large number of unruptured associated aneurysms (see section 6.3.2.). AVMs and DACA aneurysms co-existed in our material in only $1 \%$ of the patients, much less than the $3-15 \%$ described in previous reports $[52,383,438]$. This could be due to less selected patient population in our centers. 


\subsubsection{ICHs from ruptured DACA aneurysms}

The high incidence of ICHs, small aneurysm size, and high number of associated aneurysms were the special features of the ruptured DACA aneurysms in comparison to the ruptured intracranial aneurysms in general. In previous reports, an $\mathrm{ICH}$ was related to a ruptured DACA aneurysm in $17-73 \%$ of the cases $[122,172,231$, $312,368,376,383]$. In our series, $53 \%$ of patients with a ruptured DACA aneurysm had an $\mathrm{ICH}$ on the initial CT scan as compared to $25 \%$ for the ruptured aneurysms in general. The high incidence of ICHs is obviously related to the narrow pericallosal cistern and dense attachments of the aneurysm with the adjacent brain. ICHs were relatively well tolerated in patients with ruptured DACA aneurysms as they had a similar distribution of preoperative Hunt and Hess grades compared to patients with ruptured aneurysms in general. Nonetheless, ICH predicted unfavorable outcome in patients with ruptured DACA aneurysms. The presence of an expansive $\mathrm{ICH}$ in a ruptured DACA aneurysm favors clipping over coiling but at the same time makes the microneurosurgical approach much more demanding due to the lack of space and distorted anatomy.

\subsubsection{Imaging of DACA aneurysms and CTA}

Even though DSA has been the gold standard of aneurysm imaging in the past [237], during the last decade CTA has become the primary imaging method in many centers as it is noninvasive, fast, and offers similar resolution in aneurysms larger than $2 \mathrm{~mm}[165,292]$. All previous, larger series on DACA aneurysms have used DSA data for radiological analysis $[52,122,312,383]$. Our series is the first one which is largely based on CTA data in $61 \%$ of the patients. CTA seems to solve certain problems compared to planar DSA images: it gives more accurate information on the aneurysm location with respect to the corpus callosum, and with a possible $\mathrm{ICH}$. In addition, the $2 \mathrm{D}$ and $3 \mathrm{D}$ reconstructions show the exact dome orientation and base morphology with respect to the originating branches, and they also help to determine from which artery the aneurysm originates. With CTA, it is possible to evaluate all the vessels of the anterior circulation at the same time, which enables more accurate distinction between the different ACA anomalies, better evaluation of the whole ACoA complex, and easier identification of the associated aneurysms. Based on this series, we would strongly recommend either CTA or modern rotational DSA for preoperative imaging of DACA aneurysms.

\subsection{Treatment of DACA aneurysms}

\subsubsection{Clipping of DACA aneurysms}

Microsurgical clipping of DACA aneurysms presents certain specific difficulties when compared to other anterior circulation aneurysms $[52,122,142,231,248,264,279,312,383,438,443$, 445].

Yaşargil listed these special features as [443]:

i) Lack of working space in the interhemispheric space and pericallosal cistern.

ii) Dense adhesions between the cingulate gyri make separation and finding of the aneurysm difficult.

iii) Sclerotic wall and broad base of the aneurysm require precise positioning of the aneurysm clip.

iv) Origins of the branching arteries at the neck and attachment of the dome to the opposite pericallosal artery increase risk of vascular ischemic complications.

v) Difficulty in identifying lateralization of the parent artery from the preoperative images. 
vi) Attachment or embedding of the dome in the pial layer of the cingulate gyrus increases risk of tear in the aneurysm wall during dissection.

vii) With aneurysm at the bifurcation of an azygos pericallosal artery, complications may lead to vascular damage of both hemispheres.

Based on our observations, we wish to add the following features:

viii) The bridging veins cause difficulties in entering the interhemispheric fissure.

ix) Frontal expansive $\mathrm{ICH}$ results in lack of space and difficult dissection.

$x)$ Frequent intraoperative ruptures occur due to unfavorable angle of approach.

xi) Difficulty in obtaining proximal control, particularly in the region inferior to the corpus callosum.

xii) Small aneurysm size makes correct clip placement difficult.

xiii) Orientation problems in acute $\mathrm{SAH}$ with thick blood clots inside the interhemispheric fissure.

xiv) Problems in localizing the aneurysm due to lack of consistent anatomic landmarks.

Despite these challenging features, microsurgical clipping still remains the primary occlusive treatment modality for DACA aneurysms over the present techniques of endovascular therapy. In the present series, the complication rates for microsurgical treatment were similar in the ruptured and unruptured DACA aneurysms, and they did not differ from those of other aneurysm locations.

\subsubsection{Coiling of DACA aneurysms}

So far, although the published series on endovascular treatment of DACA aneurysms are small, most authors have reported more procedural difficulties than for other aneurysm locations $[184,265,291]$. In our small series of 12 coiled patients with ruptured DACA aneurysm, the success rate in total occlusions was lower than after microsurgical clipping. Our material is too small to evaluate the long-term clinical outcome after coiling of DACA aneurysms. Even with the present technology, endovascular occlusion of DACA aneurysms is still demanding because of the small size, relatively wide neck, branches originating close to the base, small caliber of parent artery, and distal location of the aneurysm. The high incidence of ICHs in patients with ruptured DACA aneurysms also favors microsurgery for immediate clot removal. In our practice we discuss each aneurysm patient in our neurovascular group and select the treatment modality for each patient individually in order to obtain the best possible overall result in the long run.

\subsection{Outcome}

\subsubsection{Ruptured DACA aneurysms}

\subsubsection{One year outcome}

Microsurgical series on patients with ruptured DACA aneurysms have reported favorable outcome in $58-83 \%$ and management mortality in $7-21 \%$ of the cases, respectively $[52,122$, $231,264,279,312,368,383,438]$. This is well in line with the $74 \%$ favorable outcome and $13 \%$ case-fatality rate in our material with complete follow-up. The Cooperative Study showed far worse results after conservative treatment of ruptured DACA aneurysms than for any other aneurysm location, with a 75\% mortality [267]. Likewise, some surgical series have suspected less favorable prognosis for patients with DACA aneurysms when compared to other aneurysm sites $[52,231]$. We did not see such a trend in our series.

Based on our results, with modern treatment methods, ruptured DACA aneurysms 
carry similar prognosis for favorable outcome as other ruptured aneurysms but their mortality rate is lower. This might be due to the frontal and relatively superficial location of DACA aneurysms. As we have observed, almost all ICHs related to ruptured DACA aneurysms are in the frontal lobes. If similar sized hematomas were located in more central regions of the brain or closer to eloquent areas, their effect on patients' outcome would probably be much more devastating. The same applies to vascular complications, e.g. ischemia related to occlusion of the distal anterior cerebral artery is probably better tolerated than ischemia in central structures. Therefore, patients with ruptured DACA aneurysms will not die of SAH as frequently as patients with other ruptured aneurysms, and they will have equally favorable outcomes as patients with other ruptured aneurysms. However, there will be more of those with severe disability among the survivors.

\subsubsection{Predictors of outcome at one year}

For patients with ruptured DACA aneurysms we found age, Hunt and Hess grade, rebleeding before treatment, ICH, IVH, and severe preoperative hydrocephalus as independent factors predicting unfavorable outcome at one year follow-up. Hunt and Hess grade was the only one of these factors also identified by previous series on DACA aneurysms [248,383]. Similarly, series on other aneurysm locations have observed the effect of the initial grade on patient outcome [287,329,342]. Among the patients with $\mathrm{H} \& \mathrm{H} \geq 3$, those with ruptured DACA aneurysms did better at one year than those with other ruptured aneurysms, with favorable outcomes of $59 \%$ and $49 \%$, respectively. Based on this finding, one might consider a more active approach towards treatment of poor grade $\mathrm{SAH}$ patients when the SAH is caused by a ruptured DACA aneurysm.

Our initial CT findings were strongly related to outcome. ICH, IVH and severe preoperative hydrocephalus all predicted unfavorable out- comes. The combined presence of these three factors was especially devastating. Previously, blood seen on the preoperative CT scan (Fisher grade $\geq 3$ ) has been shown to associate with poor outcome in patients with $\mathrm{SAH}$ in general $[287,329]$. The same seems to apply for ruptured DACA aneurysms in cases where the blood is either intraparenchymal or intraventricular. Blood clots in the subarachnoid space only (Fisher grade $=3$ ) were not associated with outcome in our series.

Although rebleeding was not a statistically significant predictor for outcome in the univariate analysis, the multivariate model revealed it was an independent risk factor. It is also the only risk factor that can be prevented by rapid neurosurgical intervention. It has become our policy to treat patients with ruptured aneurysms as soon as possible, preferably within the first 24 hours and no later than 72 hours after the initial rupture to prevent possible rebleedings [278].

\subsubsection{Neurological deficits}

DACA aneurysms are sometimes associated with certain unique neurological deficits such as akinetic mutism, bilateral leg weakness, behavioral changes and cognitive deficits $[33,71,87,99,382]$. In our series, we were not able to identify any patients with akinetic mutism or bilateral leg weakness among the patients with favorable outcome. These symptoms were probably present in some of our patients with unfavorable outcome, but may have been masked by the overall poor condition of these patients. Therefore, we were not able to determine the incidence rates of these neurological deficits in our series.

\subsubsection{Unruptured DACA aneurysms}

Clipping of unruptured DACA aneurysms was a safe and effective method in our series. The most important factor determining the outcome in these patients was the presence 
or absence of acute SAH. Clearly, patients in whom the unruptured DACA aneurysm was treated during the acute SAH phase did worse than those treated later on after recovery; the latter group had the same excellent results as patients with no history of SAH. We did not observe a similar difference in patients with $\mathrm{SAH}$ caused by a ruptured DACA aneurysm. In these patients, simultaneous treatment of associated DACA aneurysm(s) did not affect the outcome. The same also applied to patients without SAH in whom the treatment of multiple aneurysms had equally favorable outcome. Our results suggest that, in patients with acute $\mathrm{SAH}$, associated unruptured DACA aneurysm(s) should be treated only when they can be reached easily via the same microsurgical approach, i.e. only if the SAH is caused by an other DACA aneurysm. In all other cases one should wait for the patient to recover from the $\mathrm{SAH}$ and its primary treatment and then treat the unruptured DACA aneurysm in a separate session together with possible other associated aneurysms.

\subsection{Long-term follow-up}

\subsubsection{Rebleeding from clipped DACA aneu- rysms}

In our series with a median follow-up time of 10 years, there was only one rebleeding (0.4\%) at 17 years after the initial clipping and no deaths from the 262 clipped DACA aneurysms. DACA aneurysms are located relatively distally in the cerebrovascular tree with narrow parent arteries, which could indicate a smaller shear stress onto the vessel wall, making DACA aneurysms less prone to rebleeding than aneurysms at more proximal locations [427]. Based on our results microsurgical clipping is a longlasting treatment for DACA aneurysms.

\subsubsection{Recurrent SAH and de novo aneu- rysms}

During the long-term follow-up we observed four new episodes of $\mathrm{SAH}$, but only one from a DACA aneurysm. The cumulative incidence rate for recurrent $\mathrm{SAH}$ at 10 years was $1.4 \%$. This was similar to a Japanese study where the risk for $\mathrm{SAH}$ from a de novo aneurysm was $1.4 \%$ during the 10-year follow-up [409]. Only one of the 92 deaths (1\%) was due to recurrent $\mathrm{SAH}$ from a de novo or a previously undiagnosed aneurysm. De novo aneurysms have been reported to develop at a $0.84-0.89 \%$ annual rate $[161,408]$. Altogether, recurrent $\mathrm{SAH}$ seems to be a rare cause of death during a long-term follow-up in patients after surgically treated ruptured DACA aneurysms, even if the cumulative rupture rate increases with followup time and the relative risk compared to the general population is higher $[160,419]$.

\subsubsection{Mortality}

The management mortality at 12 months was $13 \%$, similar to previous studies [337,338], and most of these early deaths (92\%) were directly due to the primary $\mathrm{SAH}$ and its sequelae. Later in the follow-up, the distribution of death causes started to resemble that of the general Finnish population with two major causes of death, cardiovascular disease and cancer. Several studies have addressed the acute extracranial complications after SAH such as cardiac arrhythmias, cardiac dysfunction, myocardial injury, pulmonary edema, acute lung injury, and renal or hepatic dysfunction [377,413], but there are no studies on the long-term effect of SAH on the cardiorespiratory system. Hypertension and smoking are important risk factors for SAH but they also predispose to cardiovascular disease and cancer $[76,156]$.

\subsubsection{Excess mortality}


Relatively little is known about the longterm survival after aneurysmal SAH as only two population based studies have been published $[281,337]$. Both of these studies used the standardized mortality ratio (SMR) as a measure of excess mortality instead of the annual relative survival ratio (RSR) [69]. Unlike the SMR, which is a quotient of the observed over the expected number of deaths, the RSR takes into account the temporal variation of excess mortality [69]. This makes it a more sophisticated method for evaluation of excess mortality. In our series, the patients who survived three years from rupture of DACA aneurysm experienced no excess mortality compared to the matched population.

\subsubsection{Long-term angiographic screening}

Based on our study, routine angiographic control studies during a long-term follow-up are unlikely to be effective in preventing new episodes of SAH [161]. Young patients with multiple aneurysms, family history, and heavy smoking may need to be followed up at 5 to $10-$ year intervals. Our data supports the view that, after a recovery period, ruptured and treated DACA aneurysm should not cause any longterm effects on life expectancy. Aneurysms at other locations may behave differently. At the moment, unfortunately, we do not have such data available which would allow us to perform similar survival analysis for other aneurysm locations.

\subsection{Future trends}

Intracranial aneurysms are found in about $2 \%$ of the population, but the majority of them actually never rupture. Sadly, those that do rupture have a $50 \%$ mortality even with the best possible treatment. This indicates that the biggest advance in the treatment of this disease will be obtained by preventing aneurysms from rupturing altogether. Therefore, the future treatment of IAs should focus on several different issues, in many of which progress cannot be made without the data obtained from basic research:

1) Identification of patients with IAs. The key to prevent SAH is to identify and treat the individuals harboring IAs before they rupture. Screening with present imaging techniques is too time consuming and expensive to be used on a large scale outside of special groups known to be at high risk for IAs. In the future, once the genetic background of the IA disease is known, it may be possible to effectively identify patients at risk using a simple blood test, and these patients could then be selectively imaged with MRA or other method for possible IAs.

2) Patients with familial background. One of the high-risk groups for IAs are individuals who have relatives with IAs. In the near future, the whole genome mutation analysis may provide more insight into the genetic defect behind the high number of IAs in certain families. Identification of the defective genes could provide means to screen more effectively for possible individuals at increased risk of being carriers for IA.

3) Identification of rupture-prone aneurysms. Since most aneurysms never rupture, a special method enabling differentiation between stable and rupture-prone IAs is needed. It is evident that the aneurysm size or location is not a very accurate method. We would need better tools to evaluate the aneurysm wall type and to map the possible weak areas of the dome under increased hemodynamic stress. Results of basic research could be utilized for the development of targeted local therapy which would stabilize and reverse the degeneration and inflammation processes in the aneurysm wall. 
4) Targeted pharmaceutical therapy. Genetic studies focused on finding the gene(s) responsible for IA development should enable development of targeted pharmaceutical therapy to reverse the process. This therapy could then be used either locally to prevent aneurysm formation at defined locations or to prevent IA formation altogether via a systemic route. Development of a pharmacological intervention, if successful, is probably going to be the most important advance in IA treatment.

5) Preventive treatment of risk factors (smoking, hypertension, alcohol consumption). Risk factors associated with IAs and SAH are the same as those for cardiovascular diseases in general. Increasing awareness of healthier lifestyles and dietary habits and their relationship with cardiovascular diseases still require plenty of work on the population level.

6) Endovascular methods. Endovascular techniques will probably evolve toward exploiting much smaller-diameter, biologically active embolic materials, which will be deployed into the aneurysm with much better precision than offered by the present methods. The bioactive materials will be more durable and also have a therapeutic effect on the vessel wall. Preoperative imaging and flow-models of the various flow zones inside the aneurysm and the whole vascular tree could be used for guiding the embolic material into the proper place. The rapidly evolving nanotechnology may one day allow the neurosurgeon to navigate a tiny robot inside the vessels in order to heal the arterial wall locally. The borderline between endovascular and microneurosurgical treatment is likely to fade, and novel techniques might emerge using both approaches simultaneously.
7) Microneurosurgical techniques. The trend in microneurosurgical techniques is towards adoption of increasingly less invasive procedures, which can be performed fast and under local anesthesia. Microscopes are already being equipped with diverse new features enabling better visualization of the operative field and blood flow, but in the future they might also allow observation of the microanatomic structure of the aneurysm wall and identification of the weak areas. Development of smaller, lighter, and more flexible endoscopes with stereoscopic vision will provide new means for planning mini-invasive microsurgical approaches. Simple and fast while preserving the normal anatomy should be the key criteria.

8) Considerations for the aging population. The growing demands for active medical treatment in the aging patient population will challenge the protagonists of both endovascular and microneurosurgical treatment. Increasingly older patients with more comorbidities will ask for preventive treatment of their unruptured aneurysms. This will require special mini-invasive and fast treatment approaches not only from neurosurgeons but also from neuroanesthetists. The treatment guidelines will have to be reevaluated as well.

9) Redo aneurysms. With the increasing number of unruptured aneurysms being detected and treated, the number of treatment failures and recurrences will also increase. These redo cases of aneurysms filled with embolic material from the previous treatments will pose a special challenge. They will require a combination of endovascular and microsurgical approaches, including bypass surgery, for optimal results. Therefore, specialized neurovascular collaborative teams consisting of neurosurgeons, neuroradiologists, neuroanesthetists, and 
neurointerventionalists will be needed to take care of these patients. 


\section{Conclusions}

I. Even when ruptured, DACA aneurysms are small, and often with wide necks and narrow parent artery diameters. Almost all of them have at least one branch originating from the base. DACA aneurysms are usually located on the $A 3$ segment of the ACA, anterior to the genu of the corpus callosum. Anatomic anomalies of the ACA, especially the bihemispheric ACA, are frequently observed in association with DACA aneurysms.

II. Microsurgical clipping is a safe and effective treatment method for DACA aneurysms with similar complication rates as for aneurysms at other locations. Ruptured DACA aneurysms present with similar one-year favorable outcome as ruptured aneurysms elsewhere, but their mortality is lower. Factors predicting unfavorable outcome are advanced age, poor grade on admission, rebleeding before treatment, $\mathrm{ICH}, \mathrm{IVH}$, and severe preoperative hydrocephalus. Occlusive treatment of unruptured DACA aneurysms even smaller than $7 \mathrm{~mm}$ is justified, especially in Finland, in patients with otherwise good prognosis.

III. After surviving three years from the initial $\mathrm{SAH}$, patients with ruptured DACA aneurysms have similar survival prognosis as the matched general Finnish population. During the first three years, the excess mortality is mainly due to the primary $\mathrm{SAH}$ or its sequelae. After three years, the leading causes of death are cardiovascular disease and cancer, as in the general population. Clipping is a long-lasting treatment for DACA aneurysms with a very low rate of rebleeding.
IV.-VI. Modern microneurosurgical management of DACA aneurysms requires detailed knowledge of the microanatomy of the anterior cerebral artery and the surrounding structures. The challenge is to select the appropriate approach, locate the aneurysm deep inside the interhemispheric fissure, and to clip the neck adequately without obstructing the branching arteries at the base. Each DACA aneurysm location requires some modification to the general approach. 


\section{List of 12 supplementary videos on mi- croneurosurgery of DACA aneurysms}

The supplementary CD includes these 12 videos on microneurosurgery of DACA aneurysms:

1. Craniotomy and exposure for interhemispheric approach (DACA — video 1)

2. Craniotomy and exposure for later supraorbital (LSO) approach (DACA — video 2)

3. Clipping of proximal $A_{2}$ trunk $A_{2} A$ through $L S O$ (DACA - video 3)

4. Clipping of distal $A 2$ trunk $A 2 A$ (DACA - video 4)

5. Clipping of distal frontopolar artery $A_{2} A(D A C A-$ video 5)

6. Clipping of unruptured inferior $A 3 A(D A C A-$ video 6)

7. Clipping of unruptured inferior $A 3 A(D A C A-$ video 7$)$

8. Clipping of unruptured anterior $A 3 A(D A C A$ - video 8)

9. Clipping of ruptured anterior $A_{3} A$ (DACA — video 9)

10. Clipping of ruptured $A 4$ segment AdistA (DACA — video 10)

11. Clipping of unruptured $A 5$ segment Adist $A$ (DACA — video 11)

12. Clipping of ruptured distal CMA aneurysm (DACA — video 12)

All the videos are in QuickTime ${ }^{\circledR}$ format and require a QuickTime ${ }^{\circledR}$ player to be installed. The videos were recorded during microneurosurgical operations on DACA aneurysms by Professor Juha Hernesniemi from 2005 to 2007 at the Department of Neurosurgery, Helsinki University Central Hospital, Helsinki, Finland. Professor Hernesniemi has operated on 149 patients with 184 DACA aneurysms since 1981. 


\section{Acknowledgments}

This study was carried out at the Department of Neurosurgery at the Helsinki University Central Hospital in 2004-2008, collaborating closely with the Department of Neurosurgery at the Kuopio University Hospital and the Finnish Cancer Registry. I would like to thank the following co-workers and friends for their both direct and indirect support of my work during the past years.

Juha Hernesniemi, for his support and supervision over my work. Without his experience and invention in the field of operative microneurosurgery this study would have been meaningless. His operative skills are truly remarkable and I feel very lucky that I have had the chance to be taught by him from the first day of my neurosurgical training.

Mika Niemelä, for his excellent work as my other supervisor. He was always available, never too busy to help me with my work. He came up with the original idea for the thesis and pushed me forward, especially at times when stagnation threatened.

Juha Jääskeläinen, for teaching me how to think and write in a scientific way. Although he was not an official supervisor, his influence and impact on my whole work was that of a real supervisor. He was also the one who initially introduced me to Helsinki Neurosurgery, a thing I am still grateful for.

Juha Öhman and Timo Kumpulainen, reviewers of this thesis, for their excellent comments which very much helped in improving and completing this work.

Reza Dashti and Hanna Lehto, my closest co-workers, for their generous support, friendship and help during all stages of my work.

Matti Porras, for his outstanding expertise in analyzing the radiological images. I constantly admire his knowledge and image interpretation skill. I truly miss his kind and calm support in the OR.
Seppo Juvela, for helping me with the statistical analysis of our material. I still do not understand how anyone can fit so much knowledge of the epidemiology of neurovascular diseases into his brain.

Timo Koivisto, Antti Ronkainen and Jaakko Rinne, for providing me the high quality data from Kuopio Aneurysm Database. Without their continuous work over the past years this thesis would have been much thinner.

Johanna Seppänen and Risto Sankila, my co-workers from the Finnish Cancer Registry, for performing the excellent data analysis on the long-term mortality. Their knowledge in the field of epidemiology is truly outstanding.

Ville Kärpijoki, for all his help with videoediting and the enthusiasm regarding all the technical equipment in the OR.

Maarit Alalahti and Katariina Helin, for their technical assistance.

Marja Vajaranta, for excellent language revision of this book.

My neurosurgical colleagues from Helsinki: Riku, Leena, Minna, Aki, Atte, Miikka, Jari, Jussi, Göran, Matti S., Matti W., Juha P., EsaPekka, Anna, Johan, Johanna, Päivi, Juri, Emmanuel, Juhana, Maija, Antti P. and Pentti for their friendship and support in daily work. All my other colleagues and co-workers from the departments of Neurosurgery, Anesthesiology and Radiology at Töölö hospital. It would not be possible to do this kind of work if one did not have colleagues like you.

Riitta Hari and her team from the Low Temperature Laboratory, where I got my introduction to research work years ago. Although I later moved to clinical field and discontinued my work in your laboratory, I am still very grateful for all the time I had the chance to spend with you. It was in your laboratory that I learned most of the research methodology, proper scientific conduct, scientific writing process, and 
the value of domestic and international collaboration - all skills I had the chance to use later on for the benefit of the present research work.

All my friends from the medical faculty, especially the Cursus Paranormalis, for their long-lasting friendship over the years, all the things we have done together and all the drinking songs we have sung since the first day we met.

All my family's friends for everything they have done for me in the past.

Nora, for all the kilometers we have jogged together to empty our minds from the frustration of our research works.

Sakke, Eero, Juha, and Petri, i.e.,"Poikamieskerho", for all the years I have known you. I feel extremely lucky to have true friends like you.

Elina, for always listening to me and supporting me while I was working on this thesis.

Tomáš, my brother, for being the best possible brother one can wish for.

My parents and grandparents for believing in me, supporting me and for all their love.

My grandfather, Vilibald Vladyka, for being always my role model both professionally as well as on the personal level. I hope that one day I will be as good a man as you are.

This study was financially supported by Helsinki University Central Hospital competitive research funds (EVO grant $\mathrm{TYH}_{5204}$ ) and Duodecim.

In Helsinki, November 2008

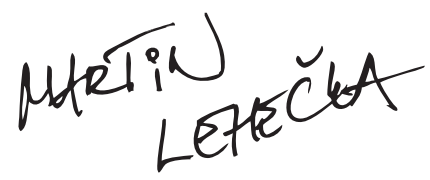


[1] Abe S, Fujii K, Nishimura K, et al. Azygos anterior cerebral artery aneurysm. Report of two cases [in Japanese]. Neurol Med Chir (Tokyo) 1985; 25(3): 215-8.

[2] Acosta C, Williams PE,Jr, Clark K. Traumatic aneurysms of the cerebral vessels. J Neurosurg 1972; 36(5): 531-6.

[3] Adams JE, Witt J. The use of otologic microscope in the surgery of aneurysms. Presented at meeting of neurosurgical society of America, January 25, 1964.

[4] Adams WM, Laitt RD, Jackson A. The role of MR angiography in the pretreatment assessment of intracranial aneurysms: a comparative study. AJNR Am J Neuroradiol 2000; 21(9): 161828.

[5] Amacher LA, Drake CG. Cerebral artery aneurysms in infancy, childhood and adolescence. Childs Brain 1975; 1(1): 72-80.

[6] Amagasa M, Onuma T, Suzuki J, et al. Traumatic anterior cerebral artery aneurysms--experiences in 4 cases and review of the literature. No Shinkei Geka 1986; 14(13): 1585-92.

[7] Anderson C, Anderson N, Bonita R, et al. Epidemiology of aneurysmal subarachnoid hemorrhage in Australia and New Zealand: incidence and case fatality from the Australasian Cooperative Research on Subarachnoid Hemorrhage Study (ACROSS). Stroke 2000; 31(8): 1843-50.

[8] Andrews BT, Dujovny M, Mirchandani HG, et al. Microsurgical anatomy of the venous drainage into the superior sagittal sinus. Neurosurgery 1989; 24(4): 514-20.

[9] Asari S, Nakamura S, Yamada O, et al. Traumatic aneurysm of peripheral cerebral arteries. Report of two cases. J Neurosurg 1977; 46(6): 795-803.

[10] Auguste KI, Ware ML, Lawton MT. Nonsaccular aneurysms of the azygos anterior cerebral artery. Neurosurg Focus 2004; 17(5): E12,1-5.

[11] Avci E, Fossett D, Aslan M, et al. Branches of the anterior cerebral artery near the anterior communicating artery complex: an anatomic study and surgical perspective. Neurol Med Chir (Tokyo) 2003; 43(7): 329-33.

[12] Avci E, Fossett D, Erdogan A, et al. Perforating branches of the anomalous anterior communicating complex. Clin Neurol Neurosurg 2001; 103(1): 19-22.

[13] Aydin IH, Onder A, Takci E, et al. Heubner's artery variations in anterior communicating artery aneurysms. Acta Neurochir (Wien) 1994; 127(1-2): 17-20.

[14] Baptista AG. Studies on the Arteries of the Brain. li. the Anterior Cerebral Artery: some Anatomic Features and their Clinical Implications. Neurology 1963; 13: 825-35.

[15] Baykal S, Ceylan S, Dinc H, et al. Aneurysm of an azygos anterior cerebral artery: report of two cases and review of the literature. Neurosurg Rev 1996; 19(1): 57-9.

[16] Becker DH, Newton TH. Distal anterior cerebral artery aneurysm. Neurosurgery 1979; 4(6): 495-503.

[17] Bederson JB, Zabramski JM, Spetzler RF. Treatment of fusiform intracranial aneurysms by circumferential wrapping with clip reinforcement. Technical note. J Neurosurg 1992; 77(3): 47880.

[18] Benedetti A, Curri D. Aneurysm of azygos anterior cerebral artery. Neurochirurgia (Stuttg) 1983; 26(2): 56-8.

[19] Benoit BG, Wortzman G. Traumatic cerebral aneurysms. Clinical features and natural history. I Neurol Neurosurg Psychiatry 1973; 36(1): 127-38.

[20] Berger PE, Harwood-Nash DC, Fitz CR. Computerized tomography: abnormal intracerebral collections of blood in children. Neuroradiology 1976; 11(1): 29-33.
[21] Biondi A, Jean B, Vivas E, et al. Giant and large peripheral cerebral aneurysms: etiopathologic considerations, endovascular treatment, and long-term follow-up. AJNR Am J Neuroradiol 2006; 27(8): 1685-92.

[22] Biumi F. Observationes anatomicae, scholiis illustrati. In: Sandifort E, ed. Thesaurus Dissertationem. Observatio V. Milan: S \& J Luchtmans; 1765:373.

[23] af Björkesten GA. Arterial aneurysms of the internal carotid artery and its bifurcation: an analysis of 69 aneurysms treated mainly by direct surgical attack. J Neurosurg 1958; 15(4): 40010.

[24] af Björkesten GA, Troupp H. Multiple intracranial arterial aneurysms. Acta Chir Scand 1960; 118: 387-91.

[25] af Björkesten GA, Troupp H. Aneurysms of the middle cerebral artery; a report on 52 cases. Acta Chir Scand 1958; 115(3): 153-9.

[26] af Björkesten GA, Troupp H. Prognosis of subarachnoid hemorrhage; a comparison between patients with verified aneurysms and patients with normal angiograms. JNeurosurg 1957; 14(4): 434-41.

[27] Blane G. History of some cases of disease in the brain, with an account of the appearance upon examination after death, and some general observations on complaints of the head. Transactions of a Society for the Improvement of Medical and Chirurgical Knowledge 1800; 2: 192-8.

[28] Bracard S, Lebedinsky A, Anxionnat R, et al. Endovascular treatment of Hunt and Hess grade IV and V aneuryms. AJNR Am J Neuroradiol 2002; 23(6): 953-7.

[29] Buncke HJ. Microsurgery--retrospective. Clin Plast Surg 1986; 13(2): 315-8.

[30] Calzolari F, Ceruti S, Pinna L, et al. Aneurysm of the azygos pericallosal artery. One case. J Neuroradiol 1991; 18(3): 27785.

[31] Carter BS, Sheth S, Chang E, et al. Epidemiology of the size distribution of intracranial bifurcation aneurysms: smaller size of distal aneurysms and increasing size of unruptured aneurysms with age. Neurosurgery 2006; 58(2): 217-23.

[32] Chhabra R, Gupta SK, Mohindra S, et al. Distal anterior cerebral artery aneurysms: bifrontal basal anterior interhemispheric approach. Surg Neurol 2005; 64(4): 315-20.

[33] Choudhari KA. Subarachnoid haemorrhage and akinetic mutism. Br J Neurosurg 2004; 18(3): 253-8.

[34] Cinnamon J, Zito J, Chalif DJ, et al. Aneurysm of the azygos pericallosal artery: diagnosis by MR imaging and MR angiography. AJNR Am J Neuroradiol 1992; 13(1): 280-2.

[35] Clare CE, Barrow DL. Infectious intracranial aneurysms. Neurosurg Clin N Am 1992; 3(3): 551-66.

[36] Cognard C, Weill A, Castaings L, et al. Intracranial berry aneurysms: angiographic and clinical results after endovascular treatment. Radiology 1998; 206(2): 499-510.

[37] Cohen JE, Rajz G, Itshayek E, et al. Endovascular management of traumatic and iatrogenic aneurysms of the pericallosal artery. Report of two cases. J Neurosurg 2005; 102(3): 555-7.

[38] Cooper A. Account of the first successful operation, performed on the common carotid artery, for aneurysm, in year 1808: with post-mortem examination, in 1821. Guy's Hosp Rep 1836; 1: 53-8.

[39] Cooper A. A case of aneurysm on the carotid artery. Med-chir Trans 1809; 1: 1-12.

[40] Cooper A. Second case of carotid aneurysm. Med-chir Trans 1809; 1: 222-33. 
[41] Cophignon J, Rey A, Thurel C, et al. Microsurgery of arterial aneurysms of the anterior part of the circle of Willis. Study of 48 consecutive cases. Neurochirurgie 1973; 19(6): 519-30.

[42] Crutchfield WG. Instruments for use in the treatment of certain intracranial vascular lesions. J Neurosurg 1959; 16(4): 471-4.

[43] Cushing $\mathrm{H}$. I. The Control of Bleeding in Operations for Brain Tumors: With the Description of Silver "Clips" for the Occlusion of Vessels Inaccessible to the Ligature. Ann Surg 1911; 54(1): 1-19.

[44] Dandy WE. Intracranial aneurysms. Ithaca: Comstock, 1944.

[45] Dandy WE. The Treatment of Internal Carotid Aneurysms within the Cavernous Sinus and the Cranial Chamber: Report of Three Cases. Ann Surg 1939; 109(5): 689-711.

[46] Dandy WE. Intracranial Aneurysm of the Internal Carotid Artery: Cured by Operation. Ann Surg 1938; 107(5): 654-9.

[47] Dashti R, Hernesniemi J, Lehto $\mathrm{H}$, et al. Microneurosurgical management of proximal anterior cerebral artery aneurysms. Surg Neurol 2007; 68(4): 366-77.

[48] Dashti R, Hernesniemi J, Niemela M, et al. Microneurosurgical management of distal middle cerebral artery aneurysms. Surg Neurol 2007; 67(6): 553-63.

[49] Dashti R, Hernesniemi J, Niemela M, et al. Microneurosurgical management of middle cerebral artery bifurcation aneurysms. Surg Neurol 2007; 67(5): 441-56.

[50] Dashti R, Rinne J, Hernesniemi J, et al. Microneurosurgical management of proximal middle cerebral artery aneurysms. Surg Neurol 2007; 67(1): 6-14.

[51] De Moullin D. Aneurysms in antiquity. Arch Chir Neerl 1961; 13: 49-63.

[52] de Sousa AA, Dantas FL, de Cardoso GT, et al. Distal anterior cerebral artery aneurysms. Surg Neurol 1999; 52(2): 128-36.

[53] Dechaume JP, Aimard G, Michel D, et al. Aneurysms of the pericallosal artery. Apropos of a series of 12 cases. Neurochirurgie 1973; 19(2): 135-50.

[54] Dickey PS, Bloomgarden GM, Arkins TJ, et al. Partial callosal resection for pericallosal aneurysms. Neurosurgery 1992; 30(1): 136-7.

[55] Dietrich W, Reinprecht A, Gruber A, et al. De novo formation and rupture of an azygos pericallosal artery aneurysm. Case report. J Neurosurg 2000; 93(6): 1062-4.

[56] Dinc C, Iplikcioglu AC, Bikmaz K. Distal anterior cerebral artery aneurysms: report of 26 cases. Neurol Med Chir (Tokyo) 2006; 46(12): 575-80.

[57] Djindjian R, Cophignon J, Theron J, et al. Embolization in vascular neuroradiology. Technic and indications apropos of 30 cases. Nouv Presse Med 1972; 1(33): 2153-8.

[58] Doby T. Cerebral angiography and Egas Moniz. AJR Am J Roentgenol 1992; 159(2): 364.

[59] Dohlman GF. Carl Olof Nylen and the birth of the otomicroscope and microsurgery. Arch Otolaryngol 1969; 90(6): 813-7.

[60] Donaghy RM. Neurosurgeon of the year: Mahmut Gazi Yaşargil. Surg Neurol 1980; 13(1): 1-3.

[61] Donaghy RM. The history of microsurgery in neurosurgery. Clin Neurosurg 1979; 26: 619-25.

[62] Dott NM. Intracranial aneurysms: cerebral arterioradiography: surgical treatment. Edinb Med J 1933; 40: 21934.

[63] Dott NM. Intracranial anurysmal formations. Clin Neurosurg 1969; 16: 1-16.

[64] Drake CG, Peerless SJ, Hernesniemi J. Surgery on vertebrobasilar aneurysms. New York: Springer-Verlag Wien, 1996.
[65] Drake CG. Surgical treatment of ruptured aneurysms of the basilar artery. Experience with 14 cases. J Neurosurg 1965; 23(5): 457-73.

[66] Duane W,Jr. A modification of the McKenzie silver clip. J Neurosurg 1950; 7(1): 92-3.

[67] Dunker RO, Harris AB. Surgical anatomy of the proximal anterior cerebral artery. J Neurosurg 1976; 44(3): 359-67.

[68] Dunn IF, Woodworth GF, Siddiqui $A H$, et al. Traumatic pericallosal artery aneurysm: a rare complication of transcallosal surgery. Case report. J Neurosurg 2007; 106/2 Suppl): 153-7.

[69] Ederer F, Axtell LM, Cutler SJ. The relative survival rate: a statistical methodology. Natl Cancer Inst Monogr 1961; 6: 101-21.

[70] Ellamushi $\mathrm{HE}$, Grieve JP, Jager HR, et al. Risk factors for the formation of multiple intracranial aneurysms. J Neurosurg 2001; 94(5): 728-32.

[71] Endo H, Shimizu H, Tominaga T. Paraparesis associated with ruptured anterior cerebral artery territory aneurysms. Surg Neurol 2005; 64(2): 135-9.

[72] Endo S, Sato S, Uneoka K, et al. Traumatic aneurysm of the callosomarginal artery: case report (author's transl). No Shinkei Geka 1974; 2(4): 329-36.

[73] Fankhauser $\mathrm{H}$, Zander E. Aneurysms of the distal anterior cerebral artery [in French]. Schweiz Arch Neurol Neurochir Psychiatr 1978; 123(2): 331-57.

[74] Farias JP, Trindade AM. Giant distal anterior cerebral artery aneurysm not visualized on angiography: case report. Surg Neurol 1997; 48(4): 348-51.

[75] Farnham JM, Camp NJ, Neuhausen SL, et al. Confirmation of chromosome $7 q 11$ locus for predisposition to intracranial aneurysm. Hum Genet 2004; 114(3): 250-5.

[76] Feigin VL, Rinkel GJ, Lawes CM, et al. Risk factors for subarachnoid hemorrhage: an updated systematic review of epidemiological studies. Stroke 2005; 36(12): 2773-80.

[77] Ferry DJ,Jr, Kempe LG. False aneurysm secondary to penetration of the brain through orbitofacial wounds. Report of two cases. J Neurosurg 1972; 36(4): 503-6.

[78] Figueiredo EG, Deshmukh P, Zabramski JM, et al. Quantitative anatomic study of three surgical approaches to the anterior communicating artery complex. Neurosurgery 2005; 56(2 Suppl): 397-405.

[79] Fischer E. Die Lageabweichungen der vorderen Hirnarterie im Gefassbild. Zentralbl Neurochir 1938; 3: 300-12.

[80] Fisher CM, Kistler JP, Davis JM. Relation of cerebral vasospasm to subarachnoid hemorrhage visualized by computerized tomographic scanning. Neurosurgery 1980; 6(1): 1-9.

[81] Fisher RG, Ciminello V. Pericallosal aneurysms. J Neurosurg 1966; 25(5): 512-5.

[82] Flamm ES. Professor M. Gazi Yaşargil: an appreciation by a former apprentice. Neurosurgery 1999; 45(5): 1015-8.

[83] Fleischer AS, Patton JM, Tindall GT. Cerebral aneurysms of traumatic origin. Surg Neurol 1975; 4(2): 233-9.

[84] Fogelholm R, Hernesniemi J, Vapalahti M. Impact of early surgery on outcome after aneurysmal subarachnoid hemorrhage. A population-based study. Stroke 1993; 24(11): 1649-54.

[85] Foutrakis GN, Yonas H, Sclabassi RJ. Saccular aneurysm formation in curved and bifurcating arteries. AJNR Am J Neuroradiol 1999; 20(7): 1309-17.

[86] Frazee JG, Cahan LD, Winter J. Bacterial intracranial aneurysms. J Neurosurg 1980; 53(5): 633-41.

[87] Freemon FR. Akinetic mutism and bilateral anterior cerebral artery occlusion. J Neurol Neurosurg Psychiatry 1971; 34(6): 693-8. 
[88] Frösen J, Piippo A, Paetau A, et al. Growth factor receptor expression and remodeling of saccular cerebral artery aneurysm walls: implications for biological therapy preventing rupture. Neurosurgery 2006; 58(3): 534-41.

[89] Frösen J, Piippo A, Paetau A, et al. Remodeling of saccular cerebral artery aneurysm wall is associated with rupture: histological analysis of 24 unruptured and 42 ruptured cases. Stroke 2004; 35(10): 2287-93.

[90] Fujimoto K, Waga S, Kojima T, et al. Aneurysm of distal anterior cerebral artery associated with azygos anterior cerebral artery. Acta Neurochir (Wien) 1981; 59(1-2): 65-9.

[91] Fujimoto Y, Yamanaka K, Nakajima Y, et al. Ruptured aneurysm arising from the proximal end of an azygos anterior cerebral artery--case report. Neurol Med Chir (Tokyo) 2004; 44(5): 242-

[92] Fukawa O, Fukada N, Aihara H, et al. Spontaneous thrombosis of an azygos anterior cerebral artery aneurysm--report of a case (author's transl). No Shinkei Geka 1982; 10(1): 105-11.

[93] Fukushima T, Miyazaki S, Takusagawa $Y$, et al. Unilateral interhemispheric keyhole approach for anterior cerebral artery aneurysms. Acta Neurochir Suppl (Wien) 1991; 53: 42-

[94] Gallagher JP. Pilojection for Intracranial Aneurysms. Report of Progress. J Neurosurg 1964; 21: 129-34.

[95] Giannotta SL, McGillicuddy JE, Kindt GW. Gradual carotid artery occlusion in the treatment of inaccessible internal carotid artery aneurysms. Neurosurgery 1979; 5(4): 417-21.

[96] Gomes F, Dujovny M, Umansky F, et al. Microsurgical anatomy of the recurrent artery of Heubner. J Neurosurg 1984; 60(1): 130-9.

[97] Gomes FB, Dujovny M, Umansky F, et al. Microanatomy of the anterior cerebral artery. Surg Neurol 1986; 26(2): 129-41.

[98] Gonzalez-Darder JM, Pesudo-Martinez JV, Feliu-Tatay RA. Microsurgical management of cerebral aneurysms based in $\mathrm{CT}$ angiography with three-dimensional reconstruction (3D(TA) and without preoperative cerebral angiography. Acta Neurochir (Wien) 2001; 143(7): 673-9.

[99] Greene KA, Marciano FF, Dickman CA, et al. Anterior communicating artery aneurysm paraparesis syndrome: clinical manifestations and pathologic correlates. Neurology 1995; 45(1): 45-50.

[100] Greenwood JJJr. Two point coagulation: a follow-up report of a new technic and instrument for electrocoagulation in neurosurgery. Arch Phys Ther 1942; 23(9): 552-4.

[101] Greenwood J,Jr. Two point caogulation: A new principle and instrument for appluing coagulation current in neurosurgery. Am J Surg 1940; 50: 267-70.

[102] Guglielmi G, Vinuela F, Dion J, et al. Electrothrombosis of saccular aneurysms via endovascular approach. Part 2: Preliminary clinical experience. J Neurosurg 1991; 75(1): 814.

[103] Guglielmi G, Vinuela F, Sepetka I, et al. Electrothrombosis of saccular aneurysms via endovascular approach. Part 1 : Electrochemical basis, technique, and experimental results. $J$ Neurosurg 1991; 75(1): 1-7.

[104] Guidetti B. Results of 98 intracranial aneurysm operations performed with the aid of an operating microscope. Acta Neurochir (Wien) 1973; 29(1): 65-71.

[105] Hackett ML, Anderson CS. Health outcomes 1 year after subarachnoid hemorrhage: An international populationbased study. The Australian Cooperative Research on Subarachnoid Hemorrhage Study Group. Neurology 2000; 55(5): 658-62.

[106] Haley EC,Jr, Kassell NF, Torner JC. The International Cooperative Study on the Timing of Aneurysm Surgery. The North American experience. Stroke 1992; 23(2): 205-14.
[107] Hamilton JG, Falconer MA. Immediate and late results of surgery in cases of saccular intracranial aneurysms. $J$ Neurosurg 1959; 16: 514-41.

[108] Handa J, Matsuda M, Koyama T, et al. Internal carotid aneurysm associated with multiple anomalies of cerebral arteries. Neuroradiology 1971; 2(4): 230-3.

[109] Harada K, Kobayashi S, Sigemori M, et al. Medullary venous malformation with azygos anterior cerebral artery aneurysm: a case report [in Japanese]. No Shinkei Geka 1987; 15(3): 327 33.

[110] Harrod CG, Bendok BR, Batjer HH. Prediction of cerebral vasospasm in patients presenting with aneurysmal subarachnoid hemorrhage: a review. Neurosurgery 2005: 56(4): 633-54

[111] Hart RG, Byer JA, Slaughter JR, et al. Occurrence and implications of seizures in subarachnoid hemorrhage due to ruptured intracranial aneurysms. Neurosurgery $1981 ; 8(4)$ 417-21.

[112] Hasan D, Vermeulen M, Wijdicks EF, et al. Management problems in acute hydrocephalus after subarachnoid hemorrhage. Stroke 1989; 20(6): 747-53.

[113] Hashizume K, Nukui H, Horikoshi T, et al. Giant aneurysm of the azygos anterior cerebral artery associated with acute subdural hematoma--case report. Neurol Med Chir (Tokyo) 1992; 32(9): 693-7.

[114] Hayashi M, Kobayashi H, Kawano H, et al. Giant aneurysm of an azygos anterior cerebral artery: report of two cases and review of the literature. Neurosurgery 1985; 17(2): 341-4.

[115] Heifetz MD. A new intracranial aneurysm clip. J Neurosurg 1969; 30(6): 753.

[116] Heiskanen O. Risks of surgery for unruptured intracranial aneurysms. J Neurosurg 1986; 65(4): 451-3.

[117] Heiskanen O, Nikki P. Large intracranial aneurysms. Acta Neurol Scand 1962; 38: 195-208.

[118] Heiskanen O, Poranen A. Surgery of incidental intracranial aneurysms. Surg Neurol 1987; 28(6): 432-6.

[119] Hernesniemi J, Dashti R, Lehecka $M$, et al. Microneurosurgical management of anterior communicating artery aneurysms. Surg Neurol 2008; 70(1): 8-29.

[120] Hernesniemi J, Ishii K, Niemelä M, et al. Lateral supraorbital approach as an alternative to the classical pterional approach. Acta Neurochir Suppl 2005; 94: 17-21.

[121] Hernesniemi J, Niemelä $M$, Karatas $A$, et al. Some collected principles of microneurosurgery: simple and fast, while preserving normal anatomy: a review. Surg Neurol 2005: 64(3): 195-200.

[122] Hernesniemi J, Tapaninaho A, Vapalahti M, et al. Saccular aneurysms of the distal anterior cerebral artery and its branches. Neurosurgery 1992; 31(6): 994-9.

[123] Hernesniemi J, Vapalahti M, Niskanen $M$, et al. One-year outcome in early aneurysm surgery: a 14 years experience. Acta Neurochir (Wien) 1993; 122(1-2): 1-10.

[124] Heros RC, Kistler JP. Intracranial arterial aneurysm--an update. Stroke 1983; 14(4): 628-31.

[125] Heros RC, Zervas NT, Varsos V. Cerebral vasospasm after subarachnoid hemorrhage: an update. Ann Neurol 1983i 14(6): 599-608

[126] Higashida RT, Halbach VV, Barnwell SL, et al. Treatment of intracranial aneurysms with preservation of the parent vessel: results of percutaneous balloon embolization in 84 patients. AJNR Am J Neuroradiol 1990; 11(4): 633-40.

[127] Higashida RT, Halbach VV, Dowd CF, et al. Intracranial aneurysms: interventional neurovascular treatment with detachable balloons-results in 215 cases. Radiology 1991; 178(3): 663-70. 
[128] Hoerenz P. Magnification: loupes and the operating microscope. Clin Obstet Gynecol 1980; 23(4): 1151-62.

[129] Hoi $\mathrm{Y}$, Meng H, Woodward SH, et al. Effects of arterial geometry on aneurysm growth: three-dimensional computational fluid dynamics study. J Neurosurg 2004; 101(4): 676-81.

[130] Hollin SA, Decker RE. Effectiveness of microsurgery for intracranial aneurysms. Postoperative angiographic study of 50 cases. J Neurosurg 1973; 39(6): 690-3.

[131] Hop JW, Rinkel GJ, Algra A, et al. Case-fatality rates and functional outcome after subarachnoid hemorrhage: a systematic review. Stroke 1997; 28(3): 660-4.

[132] Huang J, van Gelder JM. The probability of sudden death from rupture of intracranial aneurysms: a meta-analysis. Neurosurgery 2002; 51(5): 1101-7.

[133] Huber P, Braun J, Hirschmann D, et al. Incidence of berry aneurysms of the unpaired pericallosal artery: angiographic study. Neuroradiology 1980; 19(3): 143-7.

[134] Huh JS, Park SK, Shin JJ, et al. Saccular aneurysm of the azygos anterior cerebral artery: Three case reports. J Korean Neurosurg Soc 2007; 42(4): 342-5.

[135] Hunt WE, Hess RM. Surgical risk as related to time of intervention in the repair of intracranial aneurysms. J Neurosurg 1968; 28(1): 14-20.

[136] Hunter J. Works. London: Jas F. Palmer, 1835.

[137] Hussain Z, Corkill RA, Kuker W, et al. Distal aneurysms of the unpaired ACA: embryologic and therapeutic aspects. Neuroradiology 2005; 47(3): 209-14.

[138] Inagawa T. Trends in incidence and case fatality rates of aneurysmal subarachnoid hemorrhage in Izumo City, Japan, between 1980-1989 and 1990-1998. Stroke 2001; 32(7): 1499-507.

[139] Inagawa T. Effect of early operation on cerebral vasospasm. Surg Neurol 1990; 33(4): 239-46.

[140] Inagawa T. Multiple intracranial aneurysms in elderly patients. Acta Neurochir (Wien) 1990; 106(3-4): 119-26.

[141] Inagawa $T$, Kamiya K, Ogasawara $H$, et al. Rebleeding of ruptured intracranial aneurysms in the acute stage. Surg Neurol 1987; 28(2): 93-9.

[142] Inci S, Erbengi A, Özgen T. Aneurysms of the distal anterior cerebral artery: report of 14 cases and a review of the literature. Surg Neurol 1998; 50(2): 130-40.

[143] Jackson A, Fitzgerald JB, Hartley RW, et al. CT appearances of haematomas in the corpus callosum in patients with subarachnoid haemorrhage. Neuroradiology 1993; 35(6): 420-3.

[144] Jacobson JH,2nd, Suarez EL. Microsurgery in the anastomosis of small vessels. Surg Forum 1960; 11: 243-5.

[145] Jacobson JH,2nd, Wallman LJ, Schumacher GA, et al. Microsurgery as an aid to middle cerebral artery endarterectomy. J Neurosurg 1962; 19: 108-15.

[146] Jagetia A, Kumar PN, Sinha S, et al. Saccular bilobed aneurysm of an azygos anterior cerebral artery. J Clin Neurosci 2007; 14(8): 777-9.

[147] Jakobsson KE, Carlsson C, Elfverson J, et al. Traumatic aneurysms of cerebral arteries. A study of five cases. Acta Neurochir (Wien) 1984; 71(1-2): 91-8.

[148] Javid M. Urea; new use of an old agent; reduction of intracranial and intraocular pressure. Surg Clin North Am 1958; 38(4): 907-28.

[149] Javid M, Settlage P. Effect of urea on cerebrospinal fluid pressure in human subjects; preliminary report. J Am Med Assoc 1956; 160(11): 943-9.

[150] Javid MJ. A tale of two drugs: urea and chymopapain. The 1982 Percival Bailey Oration. Neurosurgery 1983; 13(2): 2113.
[151] Jefferson G. Compression of the chiasm, optic nerves, and optic tracts by intracranial aneurysms. Brain 1937; 60: 44497.

[152] Jennett B, Bond M. Assessment of outcome after severe brain damage. Lancet 1975; 1(7905): 480-4.

[153] Johnston SC, Selvin S, Gress DR. The burden, trends, and demographics of mortality from subarachnoid hemorrhage. Neurology 1998; 50(5): 1413-8.

[154] Juvela S. Treatment options of unruptured intracranial aneurysms. Stroke 2004; 35(2): 372-4.

[155] Juvela S. Prehemorrhage risk factors for fatal intracranial aneurysm rupture. Stroke 2003; 34(8): 1852-7.

[156] Juvela S. Risk factors for aneurysmal subarachnoid hemorrhage. Stroke 2002; 33(9): 2152-3.

[157] Juvela S. Risk factors for multiple intracranial aneurysms. Stroke 2000; 31(2): 392-7.

[158] Juvela S. Rebleeding from ruptured intracranial aneurysms. Surg Neurol 1989; 32(5): 323-6.

[159] Juvela S, Hillbom M, Numminen $H$, et al. Cigarette smoking and alcohol consumption as risk factors for aneurysmal subarachnoid hemorrhage. Stroke 1993; 24(5): 639-46.

[160] Juvela S, Porras M, Poussa K. Natural history of unruptured intracranial aneurysms: probability of and risk factors for aneurysm rupture. J Neurosurg 2000; 93(3): 379-87.

[161] Juvela S, Poussa K, Porras M. Factors affecting formation and growth of intracranial aneurysms: a long-term follow-up study. Stroke 2001; 32(2): 485-91.

[162] Kakou M, Destrieux C, Velut S. Microanatomy of the pericallosal arterial complex. J Neurosurg 2000; 93(4): 66775 .

[163] Kaneko M, Nukui H, Sasaki H, et al. Azygos anterior cerebral artery aneurysm [in Japanese]. Neurol Med Chir (Tokyo) 1985; 25(4): 311-6.

[164] Kanemoto $Y$, Tanaka $Y$, Nonaka M, et al. Giant aneurysm of the azygos anterior cerebral artery--case report. Neurol Med Chir (Tokyo) 2000; 40(9): 472-5.

[165] Kangasniemi M, Makela T, Koskinen S, et al. Detection of intracranial aneurysms with two-dimensional and threedimensional multislice helical computed tomographic angiography. Neurosurgery 2004; 54(2): 336-41.

[166] Kaplan HA. The lateral perforating branches of the anterior and middle cerebral arteries. J Neurosurg 1965; 23(3): 30510.

[167] Karamessini MT, Kagadis GC, Petsas T, et al. CT angiography with three-dimensional techniques for the early diagnosis of intracranial aneurysms. Comparison with intra-arterial DSA and the surgical findings. Eur J Radiol 2004; 49(3): 212-23.

[168] Karenberg A, Hort I. Medieval descriptions and doctrines of stroke: preliminary analysis of select sources. Part II: between Galenism and Aristotelism - Islamic theories of apoplexy (800-1200). J Hist Neurosci 1998; 7(3): 174-85.

[169] Karenberg A, Hort I. Medieval descriptions and doctrines of stroke: preliminary analysis of select sources. Part III: multiplying speculation - the high and late Middle Ages (1000-1450). J Hist Neurosci 1998; 7(3): 186-200.

[170] Kassell NF, Sasaki T, Colohan AR, et al. Cerebral vasospasm following aneurysmal subarachnoid hemorrhage. Stroke 1985; 16(4): 562-72.

[171] Kassell NF, Torner JC. Aneurysmal rebleeding: a preliminary report from the Cooperative Aneurysm Study. Neurosurgery 1983; 13(5): 479-81.

[172] Kassell NF, Torner JC, Haley EC,Jr, et al. The International Cooperative Study on the Timing of Aneurysm Surgery. Part 1: Overall management results. J Neurosurg 1990; 73(1): 1836. 
[173] Kassell NF, Torner JC, Jane JA, et al. The International Cooperative Study on the Timing of Aneurysm Surgery. Part 2: Surgical results. J Neurosurg 1990; 73(1): 37-47.

[174] Kasuya H, Shimizu T, Nakaya K, et al. Angeles between $A_{1}$ and $A_{2}$ segments of the anterior cerebral artery visualized by three-dimensional computed tomographic angiography and association of anterior communicating artery aneurysms. Neurosurgery 1999; 45(1): 89-94.

[175] Kataoka K, Taneda M, Asai T, et al. Structural fragility and inflammatory response of ruptured cerebral aneurysms. A comparative study between ruptured and unruptured cerebral aneurysms. Stroke 1999; 30(7): 1396-401.

[176] Katz RW, Horoupian DS, Zingesser L. Aneurysm of azygous anterior cerebral artery. A case report. J Neurosurg 1978; 48(5): 804-8.

[177] Kawamura S, Suzuki A, Sayama I, et al. Ruptured aneurysm of the distal anterior cerebral artery [in Japanese; English abstr.]. Jpn J Stroke (Tokyo) 1988; 10: 298-305.

[178] Kawashima M, Matsushima T, Sasaki T. Surgical strategy for distal anterior cerebral artery aneurysms: microsurgical anatomy. J Neurosurg 2003; 99(3): 517-25.

[179] Kawashima M, Rhoton AL,Jr, Tanriover N, et al. Microsurgical anatomy of cerebral revascularization. Part I: anterior circulation. J Neurosurg 2005; 102(1): 116-31.

[180] Keen WW. Intracranial lesions. M News New York 1890; 57: 439-49.

[181] Keogh AJ, Sharma RR, Vanner GK. Partial callosal resection for pericallosal aneurysms. Neurosurgery 1992; 31(5): 979-80.

[182] Kerber C. History of endovascular neurosurgery: a personal view. Neurosurgery 2006; 59(5 Suppl 3): S22-29.

[183] Kessler LA. Unusual anomaly of the anterior cerebral artery: report of a case. Arch Neurol 1979; 36(8): 509-10.

[184] Keston P, White PM, Horribine L, et al. The endovascular management of pericallosal artery aneurysms. J Neuroradiol 2004; 31(5): 384-90.

[185] Kim K, Mizunari T, Mizutani N, et al. Giant intracranial aneurysm of the anterior communicating artery treated by direct surgery using $\mathrm{A}_{3}-\mathrm{A}_{3}$ side-to-side anastomosis and A3-RA graft-STA anastomosis. Acta Neurochir (Wien) 2006; 148(3): 353-7.

[186] Kim LJ, Albuquerque FC, McDougall C, et al. Combined surgical and endovascular treatment of a recurrent $A_{3}-A_{3}$ junction aneurysm unsuitable for stand-alone clip ligation or coil occlusion. Technical note. Neurosurg Focus 2005; 18(2): E6.

[187] Kim LJ, Klopfenstein JD, Spetzler RF. Clip reconstruction and sling wrapping of a fusiform aneurysm: technical note. Neurosurgery 2007; 61(3 Suppl): 79-80.

[188] Kinoshita K, Matsukado Y. Aneurysms of the distal anterior cerebral artery [in Japanese; English abstr.]. Brain Nerve (Tokyo) 1975; 27: 1193-202.

[189] Kissela BM, Sauerbeck L, Woo D, et al. Subarachnoid hemorrhage: a preventable disease with a heritable component. Stroke 2002; 33(5): 1321-6.

[190] Kitamura A, Nakagawa Y, Sato M, et al. Proportions of stroke subtypes among men and women $>$ or $=40$ years of age in an urban Japanese city in 1992, 1997, and 2002. Stroke 2006; 37(6): 1374-8.

[191] Kivisaari RP, Porras M, Öhman J, et al. Routine cerebral angiography after surgery for saccular aneurysms: is it worth it? Neurosurgery 2004; 55(5): 1015-24.

[192] Kobayashi S, Yuge T, Sugita Y, et al. Azygos anterior cerebral artery aneurysm associated with fenestration of the anterior cerebral artery. Kurume Med J 1986; 33(3): 149-53.

[193] Koivisto T, Vanninen R, Hurskainen $\mathrm{H}$, et al. Outcomes of early endovascular versus surgical treatment of ruptured cerebral aneurysms. A prospective randomized study. Stroke 2000; 31(10): 2369-77.

[194] Komotar RJ, Mocco J, Solomon RA. Guidelines for the surgical treatment of unruptured intracranial aneurysms: The first annual J. Lawrence Pool memorial research symposium - Controversies in the management of cerebral aneurysms. Neurosurgery 2008; 62(1): 183-94.

[195] Kondo A, Koyama T, Ishikawa J, et al. Ruptured aneurysm of an azygos anterior cerebral artery. Neuroradiology 1979; 17(4): 227-9.

[196] Kosierkiewicz TA, Factor SM, Dickson DW. Immunocytochemical studies of atherosclerotic lesions of cerebral berry aneurysms. J Neuropathol Exp Neurol 1994; 53(4): 399-406.

[197] Koyama S. Giant aneurysm of the pericallosal artery causing acute subdural hematoma--case report. Neurol Med Chir (Tokyo) 2000; 40(5): 268-71.

[198] Krayenbühl H, Yaşargil MG. Anterior Cerebral and Pericallosal Arteries. In: Huber P, ed. Cerebral Angiography. Stuttgart: Georg Thieme Verlag; 1982:79-105.

[199] Krayenbühl HA, Yaşargil MG, Flamm ES, et al. Microsurgical treatment of intracranial saccular aneurysms. J Neurosurg 1972; 37(6): 678-86.

[200] Kriss TC, Kriss VM. History of the operating microscope: from magnifying glass to microneurosurgery. Neurosurgery 1998; 42(4): 899-908.

[201] Kumar M, Kitchen ND. Infective and traumatic aneurysms. Neurosurg Clin N Am 1998; 9(3): 577-86.

[202] Kuwabara $S$, Ishikawa $S$, Andoh $S$, et al. Aneurysms of the distal anterior cerebral artery. Report of 18 cases and review of 191 reported cases. Neurol Med Chir (Tokyo) 1984; 24(8): 580-90.

[203] Laaksamo E, Tulamo R, Baumann M, et al. Involvement of mitogen-activated protein kinase signaling in growth and rupture of human intracranial aneurysms. Stroke 2008; 39(3): 886-92.

[204] Laidlaw JD, Siu KH. Poor-grade aneurysmal subarachnoid hemorrhage: outcome after treatment with urgent surgery. Neurosurgery 2003; 53(6): 1275-82.

[205] Laitinen L, Snellman A. Aneurysms of the pericallosal artery: a study of 14 cases verified angiographically and treated mainly by direct surgical attack. J Neurosurg 1960; 17(3): 44758.

[206] Laitinen L, Troupp H. Reliability of partial ligation in the treatment of intracranial arterial aneurysm. Acta Neurol Scand 1961; 37: 62-6.

[207] Lanzino G, Kassell NF, Germanson TP, et al. Age and outcome after aneurysmal subarachnoid hemorrhage: why do older patients fare worse? I Neurosurg 1996; 85(3): 410-8.

[208] Lanzino G, Spetzler RF. Clip wrapping for partial avulsion of the aneurysm neck. Technical note. J Neurosurg 2003; 99(5): 931-2.

[209] Larson PS, Reisner A, Morassutti DJ, et al. Traumatic intracranial aneurysms. Neurosurg Focus 2000; 8(1): E4.

[210] Lath R, Vaniprasad A, Kat E, et al. Traumatic aneurysm of the callosomarginal artery. J Clin Neurosci 2002; 9(4): 466-8.

[211] Laurent JP, Cheek WR, Mims T, et al. Traumatic intracranial aneurysm in an infant: case report and review of the literature. Neurosurgery 1981; 9(3): 303-6.

[212] Lawton MT, Hamilton MG, Morcos JJ, et al. Revascularization and aneurysm surgery: current techniques, indications, and outcome. Neurosurgery 1996; 38(1): 83,92; discussion 92-4.

[213] Lawton MT, Heiserman JE, Prendergast VC, et al. Titanium aneurysm clips: Part III--Clinical application in 16 patients with subarachnoid hemorrhage. Neurosurgery 1996; 38(6): 1170-5. 
[214] Lawton MT, Ho JC, Bichard WD, et al. Titanium aneurysm clips: Part I--Mechanical, radiological, and biocompatibility testing. Neurosurgery 1996; 38(6): 1158-64.

[215] Lawton MT, Spetzler RF. Surgical strategies for giant intracranial aneurysms. Neurosurg Clin N Am 1998; 9(4): 72542.

[216] LeMay M, Gooding CA. The clinical significance of the azygos anterior cerebral artery (A.C.A.). Am J Roentgenol Radium Ther Nucl Med 1966; 98(3): 602-10.

[217] Lightfoote JB, Grusd RS, Nalls GA. Azygos anterior cerebral artery mimicking an anterior communicating artery aneurysm. AJNR Am J Neuroradiol 1989; 10(5 Suppl): S74.

[218] Linn FH, Rinkel GJ, Algra A, et al. Incidence of subarachnoid hemorrhage: role of region, year, and rate of computed tomography: a meta-analysis. Stroke 1996; 27(4): 625-9.

[219] Lippi D. An aneurysm in the Papyrus of Ebers (108, 3-9). Med Secoli 1990; 2(1): 1-4.

[220] Ljunggren B, Saveland H, Brandt L. Aneurysmal subarachnoid hemorrhage--historical background from a Scandinavian horizon. Surg Neurol 1984; 22(6): 605-16.

[221] Loman J, Myerson A. Visualization of the cerebral vessels by direct intra-carotid injection of thorium dioxide. AJR Am J Roentgenol 1936; 35: 188-95.

[222] Longstreth WT,Jr, Nelson LM, Koepsell TD, et al. Clinical course of spontaneous subarachnoid hemorrhage: a populationbased study in King County, Washington. Neurology 1993; 43(4): 712-8.

[223] Lougheed WM, Marshall BM. The diploscope in intracranial aneurysm surgery: results in 40 patients. Can J Surg 1969; 12(1): 75-82.

[224] Louw DF, Kaibara T, Sutherland GR. Aneurysm clips. J Neurosurg 2003; 98(3): 638-41.

[225] Magnus V. Aneurysm of the internal carotid artery. JAMA 1927; 88: 1712-3.

[226] Maiuri F, Corriero G, D'Amico L, et al. Giant aneurysm of the pericallosal artery. Neurosurgery 1990; 26(4): 703-6.

[227] Majoie CB, Sprengers ME, van Rooij WJ, et al. MR angiography at $3 \mathrm{~T}$ versus digital subtraction angiography in the follow-up of intracranial aneurysms treated with detachable coils. AJNR Am J Neuroradiol 2005; 26(6): 1349-56.

[228] Malis LI. Electrosurgery and bipolar technology. Neurosurgery 2006; 58(1 Suppl): ONS1-12.

[229] Malis LI. Electrosurgery. Technical note. J Neurosurg 1996; 85(5): 970-5.

[230] Malis LI. Instrumentation and techniques in microsurgery. Clin Neurosurg 1979; 26: 626-36.

[231] Mann KS, Yue CP, Wong G. Aneurysms of the pericallosalcallosomarginal junction. Surg Neurol 1984; 21(3): 261-6.

[232] Marinkovic S, Milisavljevic M,Marinkovic Z. Branches of the anterior communicating artery. Microsurgical anatomy. Acta Neurochir (Wien) 1990; 106(1-2): 78-85.

[233] Martin EM, Hummelgard AB. Traumatic aneurysms. J Neurosci Nurs 1986; 18(2): 89-94.

[234] Martines F, Blundo C, Chiappetta F. Surgical treatment of the distal anterior cerebral artery aneurysms. I Neurosurg Sci 1996; 40(3-4): 189-94.

[235] Matas R. Occlusion of large surgical arteries with removable metallic bands to test the efficiency of the collateral circulation. JAMA 1911; 56: 233-9.

[236] Matas R. Testing the efficiency of the collateral circulation as a preliminary to the occlusion of the great surgical arteries. Ann Surg 1911; 53:1-43.

[237] Mayberg MR, Batjer HH, Dacey R, et al. Guidelines for the management of aneurysmal subarachnoid hemorrhage. A statement for healthcare professionals from a special writing group of the Stroke Council, American Heart Association. Stroke 1994; 25(11): 2315-28.

[238] Mayfield FH, Kees G,Jr. A brief history of the development of the Mayfield clip. Technical note. J Neurosurg 1971; 35(1): 97100.

[239] McFadden JT. History of the operating microscope: from magnifying glass to microneurosurgery. Neurosurgery 2000; 46(2): 511.

[240] McFadden JT. Evolution of the crossed-action intracranial aneurysm clip. Technical note. J Neurosurg 1989; 71(2): 2936.

[241] McFadden JT. Aneurysm clips. J Neurosurg 1977; 46(1): 129.

[242] McFadden JT. The origin and evolutionary principals of spring forceps. Surg Gynecol Obstet 1970; 130(2): 356-68.

[243] McKenzie KG. Some minor modifications of Harvey Cushing's silver clip outfit. Surg Gynecol Obstet 1927; 45: 549-50.

[244] McKissock W, Walsh L. Subarachnoid haemorrhage due to intracranial aneurysms; results of treatment of 249 verified cases. Br Med J 1956; 2(4992): 559-65.

[245] Menovsky T, van Rooij WJ, Sluzewski M, et al. Coiling of ruptured pericallosal artery aneurysms. Neurosurgery 2002; 50(1): 11-5.

[246] Mineharu $Y$, Inoue $K$, Inoue $S$, et al. Model-based linkage analyses confirm chromosome 19q13.3 as a susceptibility locus for intracranial aneurysm. Stroke 2007; 38(4): 1174-8.

[247] Mishima K, Watanabe T, Sasaki T, et al. An infected partially thrombosed giant aneurysm of the azygos anterior cerebral artery [in Japanese]. No Shinkei Geka 1990; 18(5): 475-81.

[248] Miyazawa N, Nukui H, Yagi S, et al. Statistical analysis of factors affecting the outcome of patients with ruptured distal anterior cerebral artery aneurysms. Acta Neurochir (Wien) 2000; 142(11): 1241-6.

[249] Mocco J, Ransom ER, Komotar RJ, et al. Preoperative prediction of long-term outcome in poor-grade aneurysmal subarachnoid hemorrhage. Neurosurgery 2006; 59(3): 52938.

[250] Molyneux A, Kerr R, Stratton I, et al. International Subarachnoid Aneurysm Trial (ISAT) of neurosurgical clipping versus endovascular coiling in 2143 patients with ruptured intracranial aneurysms: a randomised trial. Lancet 2002; 360(9342): 1267-74

[251] Molyneux AJ, Kerr RS, Yu LM, et al. International subarachnoid aneurysm trial (ISAT) of neurosurgical clipping versus endovascular coiling in 2143 patients with ruptured intracranial aneurysms: a randomised comparison of effects on survival, dependency, seizures, rebleeding, subgroups, and aneurysm occlusion. Lancet 2005; 366(9488): 809-17.

[252] Moniz E. Cerebral angiography: Its application in clinical practice and physiology. Lancet 1933; 225: 1144-7.

[253] Moniz E. L'encephalographie arterielle, son importance dans la localization des temeurs cerebrales [in French]. Rev Neurol 1927; 2: 72-90.

[254] Moret J. Endovascular treatment of berry aneurysms by endosaccular occlusion. Acta Neurochir Suppl (Wien) 1991; 53: 48-9.

[255] Moret J, Cognard C, Weill A, et al. Reconstruction technic in the treatment of wide-neck intracranial aneurysms. Longterm angiographic and clinical results. Apropos of 56 cases. $J$ Neuroradiol 1997; 24(1): 30-44.

[256] Morgagni JB. De Sedibus et Causis Morborum per Anatomen Indagatis, Book 1, Letters 4 and 4, 1769.

[257] Moscow N, Michotey P, Salamon G. Anatomy of the cortical branches of the anterior cerebral artery. In: Newton TH,Potts DG, eds. Radiology of the skull and brain. vol 2, book 2. St Louis: CV Mosby; 1974:1411-20. 
[258] Nagy L, Ishii K, Karatas A, et al. Water dissection technique of Toth for opening neurosurgical cleavage planes. Surg Neurol 2006; 65(1): 38-41.

[259] Nahed BV, Seker A, Guclu B, et al. Mapping a Mendelian form of intracranial aneurysm to 1 p34.3-p36.13. Am J Hum Genet 2005; 76(1): 172-9.

[260] Naidech AM, Janjua N, Kreiter KT, et al. Predictors and impact of aneurysm rebleeding after subarachnoid hemorrhage. Arch Neurol 2005; 62(3): 410-6.

[261] Nakstad P, Nornes $H$, Hauge HN. Traumatic aneurysms of the pericallosal arteries. Neuroradiology 1986; 28(4): 335-8.

[262] Nardi PV, Esposito S, Greco R, et al. Aneurysms of azygous anterior cerebral artery. Report of two cases treated by surgery. J Neurosurg Sci 1990; 34(1): 17-20.

[263] Neff JM. A method for gradual automatic occlusion of larger blood vessels. JAMA 1911; 57: 700-8.

[264] Ng PY, Reilly PL, Brophy BP, et al. Distal anterior cerebral artery aneurysms: a clinical series. Br J Neurosurg 1998; 12(3): 20912.

[265] Nguyen TN, Raymond J, Roy D, et al. Endovascular treatment of pericallosal aneurysms. J Neurosurg 2007; 107(5): 973-6.

[266] Niizuma H, Kwak R, Uchida K, et al. Aneurysms of the azygos anterior cerebral artery. Surg Neurol 1981; 15(3): 225-8.

[267] Nishioka H. Report on the cooperative study of intracranial aneurysms and subarachnoid hemorrhage. Section VII. I. Evaluation of the conservative management of ruptured intracranial aneurysms. J Neurosurg 1966; 25(5): 574-92.

[268] Nishioka H. Results of the treatment of intracranial aneurysms by occlusion of the carotid artery in the neck. J Neurosurg 1966; 25(6): 660-82.

[269] Nitta T, Nakajima K, Maeda M, et al. Completely thrombosed giant aneurysm of the pericallosal artery: case report. $J$ Comput Tomogr 1987; 11(2): 140-3.

[270] Norlen G, Falconer M, Jefferson G, et al. The pathology, diagnosis and treatment of intracranial saccular aneurysms. Proc R Soc Med 1952; 45(5): 291-302.

[271] Norlen G, Olivecrona $H$. The treatment of aneurysms of the circle of Willis. J Neurosurg 1953; 10(4): 404-15.

[272] Nov AA, Cromwell LD. Traumatic pericallosal artery aneurysm. J Neuroradiol 1984; 11(1): 3-8.

[273] Nukui H, Aiba T. Clinical features in cases with the ruptured aneurysm of the distal anterior cerebral artery (author's transl). Neurol Med Chir (Tokyo) 1978; 18(1): 39-47.

[274] Nylen CO. The otomicroscope and microsurgery 1921-1971. Acta Otolaryngol 1972; 73(6): 453-4.

[275] Nyström S, Snellman A, Mäkelä T. Sur le traitment des anévrysmes de la "communicante arterieure." A propos d'une série de cent quarante-sept cas opéres [in French]. NeuroChirurgie 1962; 8: 414-22.

[276] Ogasawara K, Inagawa T, Yamamoto M, et al. Ruptured aneurysms of anterior cerebral arteries: A report on 18 verified cases [in Japanese; English abstr.]. Jpn J Stroke (Tokyo) 1987; 9: 304-10.

[277] Ohkuma H, Fujita S, Suzuki S. Incidence of aneurysmal subarachnoid hemorrhage in Shimokita, Japan, from 1989 to 1998. Stroke 2002; 33(1): 195-9.

[278] Öhman J, Heiskanen O. Timing of operation for ruptured supratentorial aneurysms: a prospective randomized study. J Neurosurg 1989; 70(1): 55-60.

[279] Ohno K, Monma S, Suzuki R, et al. Saccular aneurysms of the distal anterior cerebral artery. Neurosurgery 1990; 27(6): 90713.

[280] Oka K, Rhoton AL,Jr, Barry M, et al. Microsurgical anatomy of the superficial veins of the cerebrum. Neurosurgery 1985; 17(5): 711-48.
[281] Olafsson E, Hauser WA, Gudmundsson G. A population-based study of prognosis of ruptured cerebral aneurysm: mortality and recurrence of subarachnoid hemorrhage. Neurology 1997; 48(5): 1191-5.

[282] Olbert F, Kobinia G, Neumann I. Angiographic demonstration of azygos aneurysm [in French]. Ann Radiol (Paris) 1980; 23(4): 363-8.

[283] Olson JM, Vongpunsawad S, Kuivaniemi H, et al. Search for intracranial aneurysm susceptibility gene(s) using Finnish families. BMC Med Genet 2002; 3: 7.

[284] Onda H, Kasuya H, Yoneyama T, et al. Genomewide-linkage and haplotype-association studies map intracranial aneurysm to chromosome 7q11. Am J Hum Genet 2001; 69(4): 804-19.

[285] O'Neill M, Hope T, Thomson G. Giant intracranial aneurysms: diagnosis with special reference to computerised tomography. Clin Radiol 1980; 31(1): 27-39.

[286] Opeskin K. Traumatic pericallosal artery aneurysm. Am J Forensic Med Pathol 1995; 16(1): 11-6.

[287] Osawa M, Hongo K, Tanaka Y, et al. Results of direct surgery for aneurysmal subarachnoid haemorrhage: outcome of 2055 patients who underwent direct aneurysm surgery and profile of ruptured intracranial aneurysms. Acta Neurochir (Wien) 2001; 143(7): 655-64.

[288] Oshiro S, Tsugu H, Sakamoto S, et al. Ruptured aneurysm of the distal anterior cerebral artery: clinical features and surgical strategies. Neurol Med Chir (Tokyo) 2007; 47(4): 15964.

[289] Ostrowski AZ, Webster JE, Gurdjian ES. The proximal anterior cerebral artery: an anatomic study. Arch Neurol 1960; 3: 6614.

[290] Pakarinen S. Incidence, aetiology, and prognosis of primary subarachnoid haemorrhage. A study based on 589 cases diagnosed in a defined urban population during a defined period. Acta Neurol Scand 1967; 43(Suppl 29): 1-128.

[291] Pandey A, Rosenwasser RH, Veznedaroglu E. Management of distal anterior cerebral artery aneurysms: a single institution retrospective analysis (1997-2005). Neurosurgery 2007; 61(5): 909-17.

[292] Papke K, Kuhl CK, Fruth M, et al. Intracranial aneurysms: role of multidetector CT angiography in diagnosis and endovascular therapy planning. Radiology 2007; 244(2): 532-40.

[293] Park J, Hamm IS. Anterior interhemispheric approach for distal anterior cerebral artery aneurysm surgery: preoperative analysis of the venous anatomy can help to avoid venous infarction. Acta Neurochir (Wien) 2004; 146(9): 973-7.

[294] Perlmutter D, Rhoton AL,Jr. Microsurgical anatomy of the distal anterior cerebral artery. J Neurosurg 1978; 49(2): 20428.

[295] Perlmutter D, Rhoton AL,Jr. Microsurgical anatomy of the anterior cerebral-anterior communicating-recurrent artery complex. J Neurosurg 1976; 45(3): 259-72.

[296] Perneczky A, Fries G. Use of a new aneurysm clip with an inverted-spring mechanism to facilitate visual control during clip application. Technical note. J Neurosurg 1995; 82(5): 8989.

[297] Perthes G. Ueber die Ursache der Hirnstorungen nach Carotisunterbindung und uber arterienunterbindung ohne schadigung der intima [in German]. Arch F Klin Chir 1920; 114: 403.

[298] Phillips LH,2nd, Whisnant JP, O'Fallon WM, et al. The unchanging pattern of subarachnoid hemorrhage in a community. Neurology 1980; 30(10): 1034-40.

[299] Pia HW, Zierski J. Giant cerebral aneurysms: problems in treatment. In: Pia HW, Langmaid C,Zierski J, eds. Cerebral aneurysms. Advances in diagnosis and therapy. Berlin: Springer; 1979:336-42. 
[300] Pierot L, Boulin A, Castaings L, et al. Endovascular treatment of pericallosal artery aneurysms. Neurol Res 1996; 18(1): 4953.

[301] Pobereskin LH. Incidence and outcome of subarachnoid haemorrhage: a retrospective population based study. J Neurol Neurosurg Psychiatry 2001; 70(3): 340-3.

[302] Polevaya NV, Kalani MY, Steinberg GK, et al. The transition from hunterian ligation to intracranial aneurysm clips: a historical perspective. Neurosurg Focus 2006; 20(6): E3,1-7.

[303] Pool JL. Historical vignette--neurosurgery 50 years ago. Surg Neurol 1988; 29(5): 419-22.

[304] Pool JL, Colton RP. The dissecting microscope for intracranial vascular surgery. J Neurosurg 1966; 25(3): 315-8.

[305] Pool JL, Potts DG. Aneurysms and arteriovenous anomalies of the brain: diagnosis and treatment. New York: Hoeber Medical Div, Harper \& Row, 1965.

[306] Poppen JL. Specific treatment of intracranial aneurysms; experiences with 143 surgically treated patients. J Neurosurg 1951; 8(1): 75-102.

[307] Poppen JL. Ligation of the internal carotid artery in the neck; prevention of certain complications. J Neurosurg 1950; 7(6): 532-8.

[308] Pozzati E, Nuzzo G, Gaist G. Giant aneurysm of the pericallosal artery. Case report. J Neurosurg 1982; 57(4): 566-9.

[309] Prestigiacomo CJ. Historical perspectives: the microsurgical and endovascular treatment of aneurysms. Neurosurgery 2006; 59(5 Suppl 3): S39-47.

[310] Preul M, Tampieri D, Leblanc R. Giant aneurysm of the distal anterior cerebral artery: associated with an anterior communicating artery aneurysm and a dural arteriovenous fistula. Surg Neurol 1992; 38(5): 347-52.

[311] Proust F, Debono B, Hannequin D, et al. Treatment of anterior communicating artery aneurysms: complementary aspects of microsurgical and endovascular procedures. J Neurosurg 2003; 99(1): 3-14.

[312] Proust F, Toussaint $P$, Hannequin D, et al. Outcome in 43 patients with distal anterior cerebral artery aneurysms. Stroke 1997; 28(12): 2405-9.

[313] Quencer RM, Cox EV. Aneurysm of the azygos artery. Radiology 1977; 125(3): 724.

[314] Quincke H. Ueber Lumbalpunction. Berl Klin Wochenschr 1895; 32: 889-91.

[315] Raabe A, Beck J, Gerlach R, et al. Near-infrared indocyanine green video angiography: a new method for intraoperative assessment of vascular flow. Neurosurgery 2003; 52(1): 1329.

[316] Raabe A, Nakaji P, Beck J, et al. Prospective evaluation of surgical microscope-integrated intraoperative near-infrared indocyanine green videoangiography during aneurysm surgery. J Neurosurg 2005; 103(6): 982-9.

[317] Raaymakers TW, Rinkel GJ, Limburg M, et al. Mortality and morbidity of surgery for unruptured intracranial aneurysms: a meta-analysis. Stroke 1998; 29(8): 1531-8.

[318] Raimondi AJ, Yashon D, Reyes C, et al. Intracranial false aneurysms. Neurochirurgia (Stuttg) 1968; 11(6): 219-33.

[319] Raju BS, Purohit AK, Murthy SR, et al. Traumatic distal anterior cerebral artery aneurysm in a child: a case report. Neurol India 2001; 49(3): 295-8.

[320] Rand RW, Jannetta PJ. Micro-neurosurgery for aneurysms of the vertebral-basilar artery system. J Neurosurg 1967; 27(4): 330-5.

[321] Randell T, Niemelä M, Kyttä J, et al. Principles of neuroanesthesia in aneurysmal subarachnoid hemorrhage: The Helsinki experience. Surg Neurol 2006; 66(4): 382-8.

[322] Rankin J. Cerebral vascular accidents in patients over the age of 6o. Il. Prognosis. Scott Med J 1957; 2(5): 200-15.
[323] Rhoton AL,Jr. The cerebral veins. Neurosurgery 2002; 51(4 Suppl): S159-205.

[324] Rhoton AL,Jr. The supratentorial arteries. Neurosurgery 2002; 51(4 Suppl): S53-120.

[325] Rice JC. The microsurgical revolution in otolaryngology. Med $J$ Aust 1972; 2(18): 1011-4.

[326] Richling B. History of endovascular surgery: personal accounts of the evolution. Neurosurgery 2006; 59(5 Suppl 3): S30-38.

[327] Riina HA, Lemole GM,Jr, Spetzler RF. Anterior communicating artery aneurysms. Neurosurgery 2002; 51(4): 993-6.

[328] Rinkel GJ, Djibuti M, Algra A, et al. Prevalence and risk of rupture of intracranial aneurysms: a systematic review. Stroke 1998; 29(1): 251-6.

[329] Rinne J, Hernesniemi J, Niskanen M, et al. Analysis of 561 patients with 690 middle cerebral artery aneurysms: anatomic and clinical features as correlated to management outcome. Neurosurgery 1996; 38(1): 2-11.

[330] Rinne J, Hernesniemi J, Niskanen $M$, et al. Management outcome for multiple intracranial aneurysms. Neurosurgery 1995; 36(1): 31-8.

[331] Rinne J, Hernesniemi J, Puranen $M$, et al. Multiple intracranial aneurysms in a defined population: prospective angiographic and clinical study. Neurosurgery 1994; 35(5): 803-8.

[332] Rinne JK, Hernesniemi JA. De novo aneurysms: special multiple intracranial aneurysms. Neurosurgery 1993; 33(6): 981-5.

[333] Romodanov AP,Shcheglov VI. Endovascular method of excluding from the circulation saccular cerebral arterial aneurysms, leaving intact vessels patient. Acta Neurochir Suppl (Wien) 1979; 28(1): 312-5.

[334] Ronkainen A,Hernesniemi J. Subarachnoid haemorrhage of unknown aetiology. Acta Neurochir (Wien) 1992; 119(1-4): 29-34.

[335] Ronkainen $A$, Hernesniemi J, Puranen $M$, et al. Familial intracranial aneurysms. Lancet 1997; 349(9049): 380-4.

[336] Ronkainen A, Miettinen $\mathrm{H}$, Karkola K, et al. Risk of harboring an unruptured intracranial aneurysm. Stroke 1998; 29(2): 359-62.

[337] Ronkainen A, Niskanen M, Rinne J, et al. Evidence for excess long-term mortality after treated subarachnoid hemorrhage. Stroke 2001; 32(12): 2850-3.

[338] Ropper AH, Zervas NT. Outcome 1 year after SAH from cerebral aneurysm. Management morbidity, mortality, and functional status in 112 consecutive good-risk patients. J Neurosurg 1984; 60(5): 909-15.

[339] Rosengart AJ, Schultheiss KE, Tolentino J, et al. Prognostic factors for outcome in patients with aneurysmal subarachnoid hemorrhage. Stroke 2007; 38(8): 2315-21.

[340] Rosner SS, Rhoton AL,Jr, Ono M, et al. Microsurgical anatomy of the anterior perforating arteries. J Neurosurg 1984; 61(3): 468-85.

[341] Ross M. Histology. Blatimore: Williams and Wilkins, 1995.

[342] Ross N, Hutchinson PJ, Seeley H, et al. Timing of surgery for supratentorial aneurysmal subarachnoid haemorrhage: report of a prospective study. J Neurol Neurosurg Psychiatry 2002; 72(4): 480-4.

[343] Rostomily RC, Berger MS, Ojemann GA, et al. Postoperative deficits and functional recovery following removal of tumors involving the dominant hemisphere supplementary motor area. J Neurosurg 1991; 75(1): 62-8.

[344] Ruigrok YM, Rinkel GJ. Genetics of intracranial aneurysms. Stroke 2008; 39(3): 1049-55.

[345] Ruigrok YM, Wijmenga C, Rinkel GJ, et al. Genomewide linkage in a large Dutch family with intracranial aneurysms: replication of 2 loci for intracranial aneurysms to chromosome 
1p36.11-p36.13 and Xp22.2-p22.32. Stroke 2008; 39(4): 1096-102.

[346] Sadar ES, Jane JA, Lewis LW, et al. Traumatic aneurysms of the intracranial circulation. Surg Gynecol Obstet 1973; 137(1): 5967.

[347] Sadasivan B, Ma S, Dujovny M, et al. Use of experimental aneurysms to evaluate wrapping materials. Surg Neurol 1990; 34(1): 3-7.

[348] Sarti C, Tuomilehto J, Salomaa V, et al. Epidemiology of subarachnoid hemorrhage in Finland from 1983 to 1985. Stroke 1991; 22(7): 848-53.

[349] Sarwar M, Batnitzky S, Schechter MM, et al. Growing intracranial aneurysms. Radiology 1976; 120(3): 603-7.

[350] Saveland H, Hillman J, Brandt L, et al. Overall outcome in aneurysmal subarachnoid hemorrhage. A prospective study from neurosurgical units in Sweden during a 1 -year period. $J$ Neurosurg 1992; 76(5): 729-34.

[351] Schechter MM, Gutierrez-Mahoney CG D. The evolution of vertebral angiography. Neuroradiology 1973; 5(3): 157-64.

[352] Schick RM, Rumbaugh CL. Saccular aneurysm of the azygos anterior cerebral artery. AJNR Am J Neuroradiol 1989; 10(5 Suppl): S73.

[353] Schievink WI. Intracranial aneurysms. N Engl J Med 1997; 336(1): 28-40.

[354] Schorstein J. Carotid ligation in saccular intracranial aneurysms. Br J Surg 1940; 28: 50-70.

[355] Scoville WB. Miniature torsion bar spring aneurysm clip. Technical suggestions. J Neurosurg 1966; 25: 97.

[356] Seiler RW, Reulen HJ, Huber P, et al. Outcome of aneurysmal subarachnoid hemorrhage in a hospital population: a prospective study including early operation, intravenous nimodipine, and transcranial Doppler ultrasound. Neurosurgery 1988; 23(5): 598-604.

[357] Seldinger SI. Catheter replacement of the needle in percutaneous arteriography; a new technique. Acta Radiol 1953; 39(5): 368-76.

[358] Selverstone B, White JC. A new technique for gradual occlusion of the carotid artery. Arch Neurol Psychiatry 1951; 66: 246.

[359] Senegor M. Traumatic pericallosal aneurysm in a patient with no major trauma. Case report. J Neurosurg 1991; 75(3): 4757.

[360] Serbinenko FA. Balloon catheterization and occlusion of major cerebral vessels. J Neurosurg 1974; 41(2): 125-45.

[361] Serbinenko FA. Catheterization and occlusion of major cerebral vessels and prospects for the development of vascular neurosurgery. Vopr Neirokhir 1971; 35(5): 17-27.

[362] Serizawa T, Saeki N,Yamaura A. Microsurgical anatomy and clinical significance of the anterior communicating artery and its perforating branches. Neurosurgery 1997; 40(6): 1211-8.

[363] Shanelec DA. Optical principles of loupes. J Calif Dent Assoc 1992; 20(11): 25-32.

[364] Shenkin HA, Goluboff B,Haft H. The use of mannitol for the reduction of intracranial pressure in intracranial surgery. $J$ Neurosurg 1962; 19: 897-901.

[365] Shiokawa K, Tanikawa T, Satoh K, et al. Two cases of giant aneurysms arising from the distal segment of the anterior cerebral circulation [in Japanese]. No Shinkei Geka 1993; 21(5): 467-72.

[366] Siablis D, Kagadis GC, Karamessini MT, et al. Intracranial aneurysms: reproduction of the surgical view using $3 \mathrm{D}-\mathrm{CT}$ angiography. Eur J Radiol 2005; 55(1): 92-5.

[367] Sindou M, Auque J. The intracranial venous system as a neurosurgeon's perspective. Adv Tech Stand Neurosurg 2000; 26: 131-216.
[368] Sindou M, Pelissou-Guyotat I, Mertens P, et al. Pericallosal aneurysms. Surg Neurol 1988; 30(6): 434-40.

[369] Sivenius J, Tuomilehto J, Immonen-Raiha P, et al. Continuous 15-year decrease in incidence and mortality of stroke in Finland: the FINSTROKE study. Stroke 2004; 35(2): 420-5.

[370] Siwanuwatn R, Deshmukh P, Feiz-Erfan I, et al. Microsurgical anatomy of the transcallosal anterior interforniceal approach to the third ventricle. Neurosurgery 2005; 56(2 Suppl): 390-6.

[371] Skultety FM, Nishioka H. Report on cooperative study of intracranial aneurysms and subarachnoid hemorrhage: SECTION VIII, Part 2. The results of intracranial surgery in the treatment of aneurysms. J Neurosurg 1966; 25(6): 683-704.

[372] Smith RR, Parent AD. End-to-end anastomosis of the anterior cerebral artery after excision of a giant aneurysm. Case report. J Neurosurg 1982; 56(4): 577-80.

[373] Snellman A, Mäkelä T,Nyström S. Considérations sur ler anévrysmes de la région située entre les artères cérébrales antérieures. (A propos d'une série de cinquante-deux cas opérés.) [in French]. Neuro-Chirurgie 1959; 5: 143-61.

[374] Snellman A. 20 years ground work in neurosurgery [in Finnish]. Suomen Punainen Risti 1952; 28: 120-2.

[375] Snellman A. Cerebral angiography as a doagnostic method [in Finnish]. Duodecim 1939; 55: 26-46.

[376] Snyckers FD, Drake CG. Aneurysms of the distal anterior cerebral artery. A report on 24 verified cases. S AfrMed J 1973; 47(39): 1787-91.

[377] Solenski NJ, Haley EC,Jr, Kassell NF, et al. Medical complications of aneurysmal subarachnoid hemorrhage: a report of the multicenter, cooperative aneurysm study. Participants of the Multicenter Cooperative Aneurysm Study. Crit Care Med 1995; 23(6): 1007-17.

[378] Spetzler RF, Riina HA, Lemole GM,Jr. Giant aneurysms. Neurosurgery 2001; 49(4): 902-8.

[379] Statistics Finland. Death causes in Finland 2006. http:// pxweb2.stat.fi/database/StatFin/Ter/ksyyt/ksyyt_fi.asp, 2008.

[380] Stefani MA, Schneider FL, Marrone AC, et al. Anatomic variations of anterior cerebral artery cortical branches. Clin Anat 2000; 13(4): 231-6.

[381] Stegmayr B, Eriksson M, Asplund K. Declining mortality from subarachnoid hemorrhage: changes in incidence and case fatality from 1985 through 2000. Stroke 2004; 35(9): 205963.

[382] Stenhouse LM, Knight RG, Longmore BE, et al. Long-term cognitive deficits in patients after surgery on aneurysms of the anterior communicating artery. J Neurol Neurosurg Psychiatry 1991; 54(10): 909-14.

[383] Steven DA, Lownie SP,Ferguson GG. Aneurysms of the distal anterior cerebral artery: results in 59 consecutively managed patients. Neurosurgery 2007; 60(2): 227-34.

[384] Stewart CE, Lurito JT. Ruptured pericallosal aneurysm causing hemorrhage along the fornix. Neuroradiology 2002; 44(12): 993-5.

[385] Suarez EL, Jacobson JH,2nd. Results of small artery endarterectomy-microsurgical technique. Surg Forum 1961; 12: 256-7.

[386] Sugar O, Tinsley M. Aneurysm of terminal portion of anterior cerebral artery. Arch Neurol Psychiatry 1948; 60(1): 81-5.

[387] Sugita K, Hirota T, Iguchi I, et al. Comparative study of the pressure of various aneurysm clips. J Neurosurg 1976; 44(6): 723-7.

[388] Sundaram MB, Chow F. Seizures associated with spontaneous subarachnoid hemorrhage. Can J Neurol Sci 1986; 13(3): 22931. 
[389] Sundt TM,Jr, Nofzinger JD. Clip-grafts for aneurysm and small vessel surgery. 1. Repair of segmental defects with clip-grafts; laboratory studies and clinical correlations. 2. Clinical application of clip-grafts to aneurysms; technical considerations. J Neurosurg 1967; 27(6): 477-89.

[390] Suzuki J, Mizoi K, Yoshimoto T. Bifrontal interhemispheric approach to aneurysms of the anterior communicating artery. J Neurosurg 1986; 64(2): 183-90.

[391] Suzuki $Y$, Kawamata T, Matsumoto $H$, et al. Large distal anterior cerebral artery aneurysm associated with azygos anterior cerebral artery: case report [in Japanese]. No Shinkei Geka 1998; 26(10): 935-41.

[392] Tao X, Yu XJ, Bhattarai B, et al. Microsurgical anatomy of the anterior communicating artery complex in adult Chinese heads. Surg Neurol 2006; 65(2): 155-61.

[393] Tapaninaho A, Hernesniemi J, Vapalahti M, et al. Shuntdependent hydrocephalus after subarachnoid haemorrhage and aneurysm surgery: timing of surgery is not a risk factor. Acta Neurochir (Wien) 1993; 123(3-4): 118-24.

[394] Tew JM,Jr. M. Gazi Yaşargil: Neurosurgery's man of the century. Neurosurgery 1999; 45(5): 1010-4.

[395] Thompson JR, Harwood-Nash DC, Fitz CR. Cerebral aneurysms in children. Am J Roentgenol Radium Ther Nucl Med 1973; 118(1): 163-75.

[396] Timurkaynak E, Rhoton AL,Jr, Barry M. Microsurgical anatomy and operative approaches to the lateral ventricles. Neurosurgery 1986; 19(5): 685-723.

[397] Tipper G, U-King-Im JM, Price SJ, et al. Detection and evaluation of intracranial aneurysms with 16-row multislice CT angiography. Clin Radiol 2005; 60(5): 565-72.

[398] Tonnis W. Zur behandlung intrakranieller aneurysmen [in German]. Arch F Klin Chir 1936; 189: 474-6.

[399] Topsakal C, Ozveren MF, Erol FS, et al. Giant aneurysm of the azygos pericallosal artery: case report and review of the literature. Surg Neurol 2003; 60(6): 524-33.

[400] Törmä T. Aarno Snellman, the pioneer of modern neurosurgery in Finland and the founder of the Neurosurgical Clinic of Helsinki University Hospital [in Finnish]. Hippokrates (Helsinki) 1994; 11: 84-94.

[401] Törmä T. Development of neurosurgery in Finland. [in Finnish]. Duodecim 1963; 79: 196-9.

[402] Törmä T. Technic of percutaneous carotid angiography. [in Finnish]. Duodecim 1951; 67(2): 116-24.

[403] Travers B. A case of aneurysm by anastomosis in the orbit, cured by ligation of the common carotid artery. Med-chir Trans 1811; 2 : 1 .

[404] Traynelis VC, Dunker RO. Interhemispheric approach with callosal resection for distal anterior cerebral artery aneurysms. Technical note. J Neurosurg 1992; 77(3): 481-3.

[405] Troupp H, af Bjorkesten G. Results of a controlled trial of late surgical versus conservative treatment of intracranial arterial aneurysms. J Neurosurg 1971; 35(1): 20-4.

[406] Troupp $H$, Heiskanen O. Seeking for the neurosurgery of the past [in Finnish and Swedish]. Helsinki: Yliopistopaino, 2003:16.

[407] Troupp H, Laitinen L. Reliability of Partial Ligation in the Treatment of Intracranial Arterial Aneurysms. II. Acta Neurol Scand 1964; 40: 369-74.

[408] Tsutsumi K, Ueki K, Morita A, et al. Risk of aneurysm recurrence in patients with clipped cerebral aneurysms: results of longterm follow-up angiography. Stroke 2001; 32(5): 1191-4.

[409] Tsutsumi K, Ueki K, Usui M, et al. Risk of subarachnoid hemorrhage after surgical treatment of unruptured cerebral aneurysms. Stroke 1999; 30(6): 1181-4.
[410] Tubbs RS, Salter G, Elton S, et al. Sagittal suture as an external landmark for the superior sagittal sinus. J Neurosurg 2001; 94(6): 985-7.

[411] Tulamo R, Frösen J, Junnikkala S, et al. Complement activation associates with saccular cerebral artery aneurysm wall degeneration and rupture. Neurosurgery 2006; 59(5): 106977.

[412] Tulleken CA. A study of the anatomy of the anterior communicating artery with the aid of the operating microscope. Clin Neurol Neurosurg 1978; 80(3): 169-73.

[413] Tung P, Kopelnik A, Banki N, et al. Predictors of neurocardiogenic injury after subarachnoid hemorrhage. Stroke 2004; 35(2): 548-51.

[414] Türe U, Hicdonmez T, Elmaci I, et al. Giant pericallosal artery aneurysm: case report and review of the literature. Neurosurg Rev 2001; 24(2-3): 151-5.

[415] Türe U, Yaşargil MG,Krisht AF. The arteries of the corpus callosum: a microsurgical anatomic study. Neurosurgery 1996; 39(6): 1075-85.

[416] Uchino A, Nomiyama K, Takase Y, et al. Anterior cerebral artery variations detected by MR angiography. Neuroradiology 2006; 48(9): 647-52.

[417] Ugur HC, Kahilogullari G, Esmer AF, et al. A neurosurgical view of anatomical variations of the distal anterior cerebral artery: an anatomical study. J Neurosurg 2006; 104(2): 278-84.

[418] Umebayashi $Y$, Kuwayama M, Handa J, et al. Traumatic aneurysm of a peripheral cerebral artery: case report. Clin Radiol 1970; $21(1)$ : 36-8.

[419] van der Schaaf IC, Velthuis BK, Wermer MJ, et al. New detected aneurysms on follow-up screening in patients with previously clipped intracranial aneurysms: comparison with DSA or CTA at the time of SAH. Stroke 2005; 36(8): 1753-8.

[420] van der Voet M, Olson JM, Kuivaniemi $H$, et al. Intracranial aneurysms in Finnish families: confirmation of linkage and refinement of the interval to chromosome 19q13.3. Am J Hum Genet 2004; 74(3): 564-71.

[421] van Gijn J, Kerr RS, Rinkel GJ. Subarachnoid haemorrhage. Lancet 2007; 369(9558): 306-18.

[422] Villablanca JP, Hooshi P, Martin N, et al. Three-dimensional helical computerized tomography angiography in the diagnosis, characterization, and management of middle cerebral artery aneurysms: comparison with conventional angiography and intraoperative findings. $J$ Neurosurg 2002; 97(6): 1322-32.

[423] Vincentelli F, Lehman G, Caruso G, et al. Extracerebral course of the perforating branches of the anterior communicating artery: microsurgical anatomical study. Surg Neurol 1991; 35(2): 98-104.

[424] Vinuela F, Duckwiler G, Mawad M. Guglielmi detachable coil embolization of acute intracranial aneurysm: perioperative anatomical and clinical outcome in 403 patients. $J$ Neurosurg 1997; 86(3): 475-82.

[425] Wallman LJ. Raymond Madiford Peardon Donaghy. Surg Neurol 1978; 9(2): 73-5.

[426] Weir RU, Marcellus ML, Do HM, et al. Aneurysmal subarachnoid hemorrhage in patients with Hunt and Hess grade 4 or 5 : treatment using the Guglielmi detachable coil system. AJNR Am J Neuroradiol 2003; 24(4): 585-90.

[427] Wermer MJ, Greebe P, Algra A, et al. Incidence of recurrent subarachnoid hemorrhage after clipping for ruptured intracranial aneurysms. Stroke 2005; 36(11): 2394-9.

[428] Wermer MJ, van der Schaaf IC, Velthuis BK, et al. Follow-up screening after subarachnoid haemorrhage: frequency and determinants of new aneurysms and enlargement of existing aneurysms. Brain 2005; 128(10): 2421-9. 
[429] Werner SC, Blakemore AH, King BG. Aneurysm of the internal carotid artery within the skull. Wiring and electrothermic coagulation. JAMA 1941; 116: 578-82.

[430] Whisnant JP, Sacco SE, O'Fallon WM, et al. Referral bias in aneurysmal subarachnoid hemorrhage. I Neurosurg 1993; 78(5): 726-32.

[431] Wiebers DO, Whisnant JP, Huston J,3rd, et al. Unruptured intracranial aneurysms: natural history, clinical outcome, and risks of surgical and endovascular treatment. Lancet 2003; 362(9378): 103-10.

[432] Wilson CB, Christensen FK, Subrahmaniam MV. Intracranial Aneurysms at the Pericallosal Artery Bifurcation. Am Surg 1965; 31: 386-93.

[433] Wilson G, Riggs HE, Rupp C. The pathologic anatomy of ruptured cerebral aneurysms. J Neurosurg 1954; 11(2): 12834.

[434] Wintermark M, Ko NU, Smith WS, et al. Vasospasm after subarachnoid hemorrhage: utility of perfusion $C T$ and $C T$ angiography on diagnosis and management. AJNR Am J Neuroradiol 2006; 27(1): 26-34.

[435] Wintermark $M$, Uske $A$, Chalaron $M$, et al. Multislice computerized tomography angiography in the evaluation of intracranial aneurysms: a comparison with intraarterial digital subtraction angiography. J Neurosurg 2003; 98(4): 828-36.

[436] Wise BL, Chater N. The value of hypertonic mannitol solution in decreasing brain mass and lowering cerebro-spinal-fluid pressure. J Neurosurg 1962; 19: 1038-43.

[437] Wise BL, Chater N. Use of hypertonic mannitol solutions to lower cerebrospinal fluid pressure and decrease brain bulk in man. Surg Forum 1961; 12: 398-9.

[438] Wisoff JH, Flamm ES. Aneurysms of the distal anterior cerebral artery and associated vascular anomalies. Neurosurgery 1987; 20(5): 735-41.

[439] Yamada S, Utsunomiya M, Inoue K, et al. Genome-wide scan for Japanese familial intracranial aneurysms: linkage to several chromosomal regions. Circulation 2004; 110(24): 3727-33.

[440] Yamagami T, Handa H, Hashimoto N, et al. Giant aneurysm of the azygos anterior cerebral artery. Nippon Geka Hokan 1986; 55(6): 777-82.

[441] Yaşargil MG. Interhemispheric approach. In: Yaşargil MG, ed. Microneurosurgery. vol IVB, Stuttgart, New York: Georg Thieme Verlag; 1996:313-30.

[442] Yaşargil MG. Anterior Cerebral Artery Complex. In: Yaşargil MG, ed. Microneurosurgery. vol I, Stuttgart: Georg Thieme Verlag; 1984:92-128.

[443] Yaşargil MG. Distal Anterior Cerebral Artery aneurysms. In: Yaşargil MG, ed. Microneurosurgery. vol II, Stuttgart: Georg Thieme Verlag; 1984:224-31.

[444] Yaşargil MG, Smith R. Management of anurysms of anterior circulation by intracranial procedures. In: Youmans J, ed. Neurological surgery. vol III, Philadelphia: WB Saunders; 1982:1663-96.

[445] Yaşargil MG, Carter LP. Saccular aneurysms of the distal anterior cerebral artery. J Neurosurg 1974; 40(2): 218-23.

[446] Yaşargil MG, Fox JL. The microsurgical approach to intracranial aneurysms. Surg Neurol 1975; 3(1): 7-14.

[447] Yaşargil MG, Vise WM, Bader DC. Technical adjuncts in neurosurgery. Surg Neurol 1977; 8(5): 331-6.

[448] Yasui N, Suzuki A, Nishimura H, et al. Long-term follow-up study of unruptured intracranial aneurysms. Neurosurgery 1997; 40(6): 1155-60.

[449] Yoshimoto T, Uchida K, Suzuki J. Surgical treatment of distal anterior cerebral artery aneurysms. J Neurosurg 1979; 50(1): 40-4.
[450] Yuge $\mathrm{T}$, Shigemori M, Tokutomi $\mathrm{T}$, et al. Diffuse axonal injury associated with multiple traumatic aneurysms of the distal anterior cerebral artery--case report. Neurol Med Chir (Tokyo) 1990; 30(6): 412-6.

[451] Zacharia BE, Mocco J, Komotar RJ, et al. J. Lawrence Pool, M.D.: a pioneer in vascular neurosurgery. Neurosurg Focus 2006; 20(6): E2,1-3.

[452] Zderkiewicz E, Czochra M, Mosiewicz A. Aneurysm of the azygos anterior cerebral artery [in Polish]. Neurol Neurochir Pol 1992; 26(1): 121-6. 